\title{
Restricting unipotent characters in special orthogonal groups
}

\author{
Frank Himstedt and Felix Noeske
}

\begin{abstract}
For all prime powers $q$ we restrict the unipotent characters of the special orthogonal groups $\mathrm{SO}_{5}(q)$ and $\mathrm{SO}_{7}(q)$ to a maximal parabolic subgroup. We determine all irreducible constituents of these restrictions for $\mathrm{SO}_{5}(q)$ and a large part of the irreducible constituents for $\mathrm{SO}_{7}(q)$.
\end{abstract}

\section{Introduction}

Among the ordinary irreducible characters of a finite group $G$ of Lie type the unipotent characters possess some remarkable properties. For example, the Jordan decomposition of characters gives a connection between the ordinary irreducible characters of $G$ and the unipotent characters of certain subgroups of the dual group. Furthermore, if $\ell$ is a prime different from the defining characteristic and not too small, then the reductions modulo $\ell$ of the unipotent irreducible characters form a so-called basic set for the unipotent $\ell$-blocks of $G$, so that knowledge of the decomposition numbers of the unipotent irreducible characters can be used to derive all decomposition numbers of the unipotent blocks.

The analysis of the restriction of representations to maximal parabolic subgroups is an important tool in the representation theory of finite groups of Lie type. One reason for this is that maximal parabolic subgroups are large subgroups and another one is that the Levi decomposition in conjunction with Clifford theory often allows for a reduction of representation theoretical questions to groups of smaller rank.

Let $q$ be a prime power. In this paper we study the restriction of the unipotent irreducible characters of the special orthogonal groups $G=\mathrm{SO}_{5}(q)$ and $G=\mathrm{SO}_{7}(q)$ to the maximal parabolic subgroup $P$, which is defined as the stabilizer in $G$ of a one-dimensional subspace of the natural module. The irreducible characters of the parabolic subgroup $P$ are partitioned into Types $1,0,+$ and - via Clifford theory. For $G=\mathrm{SO}_{5}(q)$ we determine all irreducible constituents of the restrictions of the unipotent irreducible characters of $G$ to $P$. For $G=$ $\mathrm{SO}_{7}(q)$ we obtain complete information on the irreducible constituents of Types 1 and 0 and partial information on the constituents of Types \pm . Our motivation lies in the computation of the decomposition numbers of $\mathrm{SO}_{7}(q)$ in non-defining characteristic. The results we obtain in this paper contribute to solving this task in [8]. For even $q$ the special orthogonal groups are isomorphic to symplectic groups and in this case restrictions of representations in non-defining characteristic to maximal subgroups were previously investigated in $[\mathbf{7}]$ and [12], for example.

Based on our motivation to compute and compare the decomposition numbers of $\operatorname{Sp}_{6}(q)$ and $\mathrm{SO}_{7}(q)$, this paper and the paper by An and Hiss [2] are kindred spirits. In fact, large parts of our paper are guided by the work of An and Hiss. However, there are some remarkable differences: for the orthogonal groups the unipotent radical of $P$ is abelian, while it is non-abelian for the parabolic subgroup considered in [2]. Furthermore, there are structural differences between the inertia subgroups in the parabolic subgroup of the orthogonal and the symplectic groups, respectively. And, finally, we also consider even prime powers $q$.

Received 24 September 2013; revised 24 January 2015.

2010 Mathematics Subject Classification 20C15, 20C33 (primary). 
The paper is structured as follows: we fix notation for the orthogonal groups in $\S 2$ and introduce the maximal parabolic subgroup $P$. In $\S 3$ we describe the construction and parameterization of the irreducible characters of $P$ via Clifford theory. Section 4 is the technical heart of this paper. In Theorem 4.3 we obtain a description of the restriction of those characters of $G$ which are Harish-Chandra induced from the standard Levi subgroup of $P$. In most cases this reduces the decomposition of the Harish-Chandra induced character to a similar problem for smaller subgroups. In $\S 5$ we collect some general results on the restriction of the Steinberg character. Section 6 deals with the values of the unipotent irreducible characters of the special orthogonal groups on certain unipotent conjugacy classes. We show that these values already determine the degrees of the components of Types $1,0,+$ and - of the restrictions of the unipotent characters to $P$. The main results of this paper are contained in $\delta \S 7$ and 8 , where we consider the restrictions of the unipotent irreducible characters of $G=\mathrm{SO}_{5}(q)$ and $G=\mathrm{SO}_{7}(q)$ to the maximal parabolic subgroup $P$. Since $\mathrm{SO}_{7}(q)$ does not have any cuspidal unipotent character, the crucial tool is the description of the restriction of Harish-Chandra induced modules in Theorem 4.3.

\section{Special orthogonal groups}

In this section we collect some information on the special orthogonal groups $\mathrm{SO}_{n}(q)$ for odd $n$ and on a maximal parabolic subgroup $P_{n}$ of $\mathrm{SO}_{n}(q)$. For more information on orthogonal groups, we refer to [14, Chapter 11].

\subsection{Special orthogonal groups}

Let $q$ be a power of a prime $p$ and $\mathbb{F}_{q}$ a finite field with $\left|\mathbb{F}_{q}\right|=q$. We fix a positive integer $m$. Let $I_{m} \in \mathbb{F}_{q}^{m \times m}$ be the identity matrix and $J_{m} \in \mathbb{F}_{q}^{m \times m}$ the matrix with ones on the anti-diagonal and zeros elsewhere. We write $\mathbf{v} \in \mathbb{F}_{q}^{2 m+1}, \mathbf{w} \in \mathbb{F}_{q}^{2 m}$ as

$$
\mathbf{v}=\left[v_{m}, \ldots, v_{1}, v_{0}, v_{1}^{\prime}, \ldots, v_{m}^{\prime}\right]^{\operatorname{tr}}, \quad \mathbf{w}=\left[w_{m}, \ldots, w_{1}, w_{1}^{\prime}, \ldots, w_{m}^{\prime}\right]^{\operatorname{tr}} .
$$

Fix an element $\nu \in \mathbb{F}_{q}$ such that the polynomial $X^{2}+X+\nu \in \mathbb{F}_{q}[X]$ is irreducible. We define quadratic forms $Q_{2 m+1}$ on $\mathbb{F}_{q}^{2 m+1}$ and $Q_{2 m}^{ \pm}$on $\mathbb{F}_{q}^{2 m}$ by

$$
Q_{2 m+1}(\mathbf{v}):=v_{0}^{2}+\sum_{j=1}^{m} v_{j} v_{j}^{\prime}, \quad Q_{2 m}^{+}(\mathbf{w}):=\sum_{j=1}^{m} w_{j} w_{j}^{\prime}
$$

and

$$
Q_{2 m}^{-}(\mathbf{w}):=w_{1}^{2}+w_{1} w_{1}^{\prime}+\nu w_{1}^{\prime 2}+\sum_{j=2}^{m} w_{j} w_{j}^{\prime} .
$$

Let $\left\{e_{m}, \ldots, e_{1}, e_{0}, e_{1}^{\prime}, \ldots, e_{m}^{\prime}\right\}$ be the standard basis of the vector space $\mathbb{F}_{q}^{2 m+1}$ and let $\left\{f_{m}, \ldots, f_{1}, f_{1}^{\prime}, \ldots, f_{m}^{\prime}\right\}$ be the standard basis of $\mathbb{F}_{q}^{2 m}$. We set

$$
J_{2 m+1}^{\prime}:=\left[\begin{array}{ccc}
\cdot & \cdot & J_{m} \\
\cdot & 2 & \cdot \\
J_{m} & \cdot & \cdot
\end{array}\right], \quad J_{2 m}^{+}:=J_{2 m}, \quad J_{2 m}^{-}:=\left[\begin{array}{cccc}
\cdot & \cdot & \cdot & J_{m-1} \\
\cdot & 2 & 1 & \cdot \\
\cdot & 1 & 2 \nu & \cdot \\
J_{m-1} & \cdot & \cdot & \cdot
\end{array}\right] .
$$

Throughout the paper we usually write dots for zeros as matrix entries, or omit them to increase legibility. For all $\mathbf{v}, \mathbf{v}^{\prime} \in \mathbb{F}_{q}^{2 m+1}$ and $\mathbf{w}, \mathbf{w}^{\prime} \in \mathbb{F}_{q}^{2 m}$, we have

$$
\begin{aligned}
\mathbf{v}^{\operatorname{tr}} J_{2 m+1}^{\prime} \mathbf{v}^{\prime} & =Q_{2 m+1}\left(\mathbf{v}+\mathbf{v}^{\prime}\right)-Q_{2 m+1}(\mathbf{v})-Q_{2 m+1}\left(\mathbf{v}^{\prime}\right), \\
\mathbf{w}^{\operatorname{tr}} J_{2 m}^{ \pm} \mathbf{w}^{\prime} & =Q_{2 m}^{ \pm}\left(\mathbf{w}+\mathbf{w}^{\prime}\right)-Q_{2 m}^{ \pm}(\mathbf{w})-Q_{2 m}^{ \pm}\left(\mathbf{w}^{\prime}\right) .
\end{aligned}
$$


Hence, $J_{2 m+1}^{\prime}$ is the Gram matrix of the polar form of $Q_{2 m+1}$ with respect to the standard basis of $\mathbb{F}_{q}^{2 m+1}$, and $J_{2 m}^{ \pm}$is the Gram matrix of the polar form of $Q_{2 m}^{ \pm}$with respect to the standard basis of $\mathbb{F}_{q}^{2 m}$; see [14, Chapter 11]. We define

$$
\begin{aligned}
\mathrm{GO}_{2 m+1}(q) & :=\left\{\mathbf{x} \in \mathrm{GL}_{2 m+1}(q) \mid Q_{2 m+1}(\mathbf{x v})=Q_{2 m+1}(\mathbf{v}) \forall \mathbf{v} \in \mathbb{F}_{q}^{2 m+1}\right\}, \\
\mathrm{GO}_{2 m}^{ \pm}(q) & :=\left\{\mathbf{x} \in \mathrm{GL}_{2 m}(q) \mid Q_{2 m}^{ \pm}(\mathbf{x w})=Q_{2 m}^{ \pm}(\mathbf{w}) \forall \mathbf{w} \in \mathbb{F}_{q}^{2 m}\right\}, \\
\mathrm{SO}_{2 m+1}(q) & :=\operatorname{GO}_{2 m+1}(q) \cap \mathrm{SL}_{2 m+1}(q), \quad \mathrm{SO}_{2 m}^{ \pm}(q):=\mathrm{GO}_{2 m}^{ \pm}(q) \cap \mathrm{SL}_{2 m}(q),
\end{aligned}
$$

and use the convention $\mathrm{GO}_{0}^{ \pm}(q):=\mathrm{SO}_{0}^{ \pm}(q):=\{1\}$. The orders of these groups are

$$
\begin{aligned}
\left|\mathrm{GO}_{2 m+1}(q)\right| & =d \cdot q^{m^{2}}\left(q^{2 m}-1\right)\left(q^{2 m-2}-1\right) \ldots\left(q^{2}-1\right), \\
\left|\mathrm{SO}_{2 m+1}(q)\right| & =q^{m^{2}}\left(q^{2 m}-1\right)\left(q^{2 m-2}-1\right) \ldots\left(q^{2}-1\right), \\
\left|\mathrm{GO}_{2 m}^{ \pm}(q)\right| & =2 q^{m(m-1)}\left(q^{m} \mp 1\right)\left(q^{2 m-2}-1\right)\left(q^{2 m-4}-1\right) \ldots\left(q^{2}-1\right), \\
\left|\mathrm{SO}_{2 m}^{ \pm}(q)\right| & =\frac{2}{e} \cdot q^{m(m-1)}\left(q^{m} \mp 1\right)\left(q^{2 m-2}-1\right)\left(q^{2 m-4}-1\right) \ldots\left(q^{2}-1\right),
\end{aligned}
$$

where $d:=\operatorname{gcd}(2, q-1)$ and $e:=\operatorname{gcd}\left(2, q^{m} \mp 1\right)$. Note that for even $q$, we have $\operatorname{GO}_{2 m+1}(q)=$ $\mathrm{SO}_{2 m+1}(q)$ and $\mathrm{GO}_{2 m}^{ \pm}(q)=\mathrm{SO}_{2 m}^{ \pm}(q)$. This agrees with the definition in the ATLAS [4], but it differs from the one in [14].

REMARK 1. For even $q$ there is a natural isomorphism

$$
\mathrm{SO}_{2 m+1}(q) \rightarrow \mathrm{Sp}_{2 m}(q):=\left\{\mathbf{x} \in \mathrm{GL}_{2 m}(q) \mid \mathbf{x}^{\mathrm{tr}} J_{2 m} \mathbf{x}=J_{2 m}\right\}
$$

mapping each matrix $A$ to the matrix which is obtained from $A$ by removing the middle row and the middle column.

\subsection{The Weyl group}

From now on we fix an odd integer $n=2 m+1(m \geqslant 1)$ and set $G:=G_{n}:=\operatorname{SO}_{n}(q)$. The Weyl group $W$ of $G$ is of type $B_{m}$ and we number the simple roots such that the Dynkin diagram is

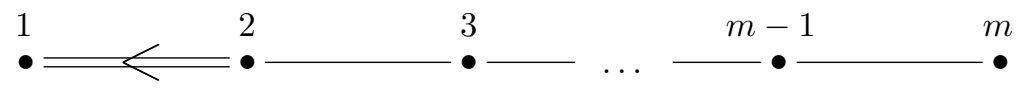

So, the first simple root is short, the others are long. Let $s_{j} \in W$ be the reflection at the $j$ th simple root, $j=1,2, \ldots, m$. An inverse image (which we also denote by $s_{j}$ ) in the normalizer $N_{G}(T)$ of the maximally split torus

$$
T:=\left\{\operatorname{diag}\left(t_{m}, \ldots, t_{1}, 1, t_{1}^{-1}, \ldots, t_{m}^{-1}\right) \mid t_{1}, \ldots, t_{m} \in \mathbb{F}_{q}^{\times}\right\}
$$

of $G$ is given by

$$
s_{1}:=\left[\begin{array}{ccccc}
I_{m-1} & & & & \\
& \cdot & \cdot & 1 & \\
& \cdot & -1 & \cdot & \\
& 1 & \cdot & \cdot & \\
& & & & I_{m-1}
\end{array}\right]
$$

and

$$
s_{j}:=\left[\begin{array}{ccccc}
I_{m-j} & & & & \\
& J_{2} & & & \\
& & I_{2 j-3} & & \\
& & & J_{2} & \\
& & & & I_{m-j}
\end{array}\right] \quad \text { for } j \in\{2,3, \ldots, m\} .
$$


For $m \geqslant 2$ we set $s:=s_{m}$ and $t:=s_{m} s_{m-1} \ldots s_{2} s_{1} s_{2} \ldots s_{m-1} s_{m}$. It follows from (2.1)-(2.3) that we can choose the following inverse images of $s$ and $t$ in $N_{G}(T)$ :

$$
s=\left[\begin{array}{lll}
J_{2} & & \\
& I_{n-4} & \\
& & J_{2}
\end{array}\right] \text { and } t=\left[\begin{array}{cccc} 
& & & \\
& I_{m-1} & \cdot & \cdot \\
& \cdot & -1 & \cdot \\
1 & \cdot & \cdot & I_{m-1}
\end{array}\right] .
$$

\subsection{A parabolic subgroup}

Let $P_{n}$ denote the stabilizer in $G_{n}$ of the one-dimensional subspace generated by the vector $e_{m}=[1,0,0, \ldots, 0]^{\text {tr }}$ in the natural module for $G_{n}$. Then $P_{n}$ is a maximal parabolic subgroup of order $\left|P_{n}\right|=q^{m^{2}}(q-1)\left(q^{2 m-2}-1\right)\left(q^{2 m-4}-1\right) \ldots\left(q^{2}-1\right)$ with Levi decomposition $P_{n}=U_{n} \rtimes L_{n}$, where

$$
L_{n}=\left\{\mathbf{s}_{n}(\mathbf{x}, a) \mid \mathbf{x} \in G_{n-2}, a \in \mathbb{F}_{q}^{\times}\right\}, \quad U_{n}=\left\{\mathbf{u}_{n}(\mathbf{v}) \mid \mathbf{v} \in \mathbb{F}_{q}^{n-2}\right\}
$$

and

$$
\mathbf{s}_{n}(\mathbf{x}, a):=\left[\begin{array}{lll}
a & & \\
& \mathbf{x} & \\
& & a^{-1}
\end{array}\right], \quad \mathbf{u}_{n}(\mathbf{v}):=\left[\begin{array}{ccc}
1 & -\mathbf{v}^{\operatorname{tr}} J_{n-2}^{\prime} & -Q_{n-2}(\mathbf{v}) \\
& 1 & \mathbf{v} \\
& & 1
\end{array}\right]
$$

The Levi subgroup $L_{n}$ is a direct product $L_{n}=L_{n}^{\prime} \times A \cong \mathrm{SO}_{n-2}(q) \times \mathbb{F}_{q}^{\times}$of

$$
L_{n}^{\prime}:=\left\{\mathbf{s}_{n}(\mathbf{x}, 1) \mid \mathbf{x} \in \mathrm{SO}_{n-2}(q)\right\} \quad \text { and } \quad A:=\left\{\mathbf{s}_{n}\left(I_{n-2}, a\right) \mid a \in \mathbb{F}_{q}^{\times}\right\} .
$$

The unipotent radical $U_{n}$ of $P_{n}$ is an elementary abelian group of order $q^{n-2}$. Often we consider $G_{n-2}$ as a subgroup of $G_{n}$ and $P_{n-2}$ as a subgroup of $L_{n}^{\prime}$ via the identification $\mathbf{x} \mapsto \mathbf{s}_{n}(\mathbf{x}, 1)$.

3. The irreducible characters of $P_{n}$

In this section we fix notation and classify the irreducible characters of the maximal parabolic subgroup $P_{n}$ via Clifford theory. A similar classification was also obtained by Schmölzer in [13, Chapter 2].

\subsection{General character theoretic notation}

Let $K$ be a subgroup of a finite group $H$. We write $\operatorname{Irr}(H)$ for the set of complex irreducible characters of $H$ and $1_{H}$ for the trivial character. Let $\langle\cdot, \cdot\rangle_{H}$ be the usual scalar product on the space of class functions of $H$. If $\chi$ is a character of $H$, we write $\chi \downarrow_{K}^{H}$ for the restriction of $\chi$ to $K$ and, if $\varphi$ is a character of $K$, we write $\varphi \uparrow_{K}^{H}$ for the character of $H$ which is induced by $\varphi$. If $K \unlhd H$ and $\psi$ is a character of the factor group $H / K$, then we denote its inflation to $H$ by $\operatorname{Infl}_{H / K}^{H} \psi$. For two characters $\chi, \psi$ of $H$ we say that $\psi$ is a subcharacter of $\chi$ if $\chi-\psi$ is a character.

\subsection{Inertia subgroups of $P_{n}$}

Let $n \geqslant 5$. The conjugation action of $L_{n}$ on $U_{n}$ is given by $\mathbf{s}_{n}(\mathbf{x}, a) \mathbf{u}_{n}(\mathbf{v})=\mathbf{u}_{n}(a \mathbf{x v})$. As before, we write $\mathbf{v} \in \mathbb{F}_{q}^{n-2}$ as $\mathbf{v}=\left[v_{m-1}, \ldots, v_{1}, v_{0}, v_{1}^{\prime}, \ldots, v_{m-1}^{\prime}\right]^{\text {tr }}$ and fix a non-trivial irreducible complex character $\xi$ of $\left(\mathbb{F}_{q},+\right)$. The corresponding action of $L_{n}$ on $\operatorname{Irr}\left(U_{n}\right)$ has four orbits. We choose as representatives $1_{U}$ (the trivial character) and non-trivial $\lambda^{0}, \lambda^{+}$and $\lambda^{-}$. 
For $\varepsilon \in\{0,+,-\}$ we denote the inertia subgroup of $\lambda^{\varepsilon}$ in $P_{n}$ by $I^{\varepsilon}$. We define $\lambda^{0}$ by $\lambda^{0}\left(\mathbf{u}_{n}(\mathbf{v})\right):=\xi\left(v_{m-1}^{\prime}\right)$ and get $I^{0}=U_{n} \tilde{P}_{n-2}$ with $\tilde{P}_{n-2}:=U_{n-2} \tilde{L}_{n-2}$, where

$$
\tilde{L}_{n-2}:=\left\{\left[\begin{array}{cccc}
a & & & \\
& a & & \\
& \mathbf{x} & & \\
& & a^{-1} & \\
& & & a^{-1}
\end{array}\right] \mid \mathbf{x} \in \operatorname{SO}_{n-4}(q), a \in \mathbb{F}_{q}^{\times}\right\}
$$

and

$$
U_{n-2}=\left\{\left[\begin{array}{lll}
1 & & \\
& \mathbf{u}_{n-2}(\mathbf{v}) & \\
& & 1
\end{array}\right] \mid \mathbf{v} \in \mathbb{F}_{q}^{n-4}\right\} \text {. }
$$

Here, we consider $U_{n-2} \leqslant G_{n-2}$ as a subgroup of $G_{n}$ as described at the end of $\S 2.3$. Furthermore, $\left|\tilde{P}_{n-2}\right|=q^{(m-1)^{2}}(q-1)\left(q^{2 m-4}-1\right)\left(q^{2 m-6}-1\right) \ldots\left(q^{2}-1\right)$. We define $\lambda^{+}$by $\lambda^{+}\left(\mathbf{u}_{n}(\mathbf{v})\right):=\xi\left(v_{0}\right)$ and so $I^{+}=U_{n} L_{n}^{+}$, where

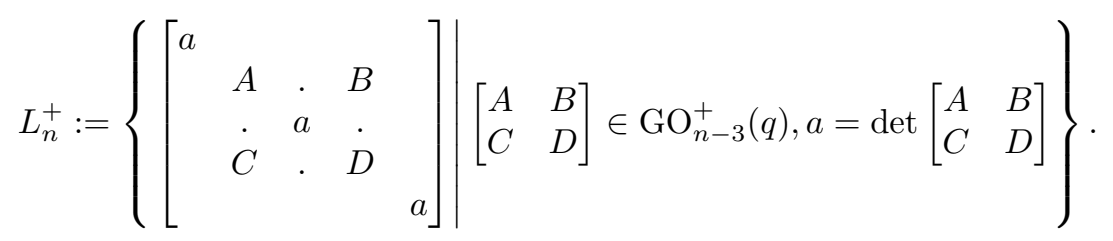

Note that $a=a^{-1}= \pm 1$. In particular, $L_{n}^{+} \cong \mathrm{GO}_{n-3}^{+}(q)$ and

$$
\left|L_{n}^{+}\right|=2 q^{(m-1)(m-2)}\left(q^{m-1}-1\right)\left(q^{2 m-4}-1\right)\left(q^{2 m-6}-1\right) \ldots\left(q^{2}-1\right) .
$$

The construction of $\lambda^{-}$is more complicated and less explicit. We define a quadratic form $Q_{n}^{\prime}$ on $\mathbb{F}_{q}^{n}$ by

$$
Q_{n}^{\prime}(\mathbf{v}):=v_{0}^{2}+v_{1}^{2}+v_{1} v_{1}^{\prime}+\nu v_{1}^{\prime 2}+\sum_{j=2}^{m} v_{j} v_{j}^{\prime} .
$$

It follows from $[\mathbf{1 4}$, p. 139, II $]$ that there are $\nu^{\prime} \in \mathbb{F}_{q}^{\times}$and

$$
\mathbf{b}_{n}=\left[\begin{array}{ccc}
\nu^{\prime} I_{m-1} & & \\
& \mathbf{b}_{3}^{\prime} & \\
& & I_{m-1}
\end{array}\right] \in \operatorname{GL}_{n}(q)
$$

such that $Q_{n}^{\prime}\left(\mathbf{b}_{n} \mathbf{v}\right)=\nu^{\prime} \cdot Q_{n}(\mathbf{v})$ for all $\mathbf{v} \in \mathbb{F}_{q}^{n}$. Note that the matrix $\mathbf{b}_{3}^{\prime} \in \mathrm{GL}_{3}(q)$ depends on $q$ but not on $n$. For odd $q$ we can choose $\nu^{\prime}$ to be 1 or a non-square in $\mathbb{F}_{q}^{\times}$; for even $q$ we can always choose $\nu^{\prime}=1$. We define $\lambda^{-}\left(\mathbf{u}_{n}(\mathbf{v})\right):=\xi\left(\left(\mathbf{b}_{n-2} \mathbf{v}\right)_{0}\right)$. A straightforward calculation shows that $\mathbf{s}_{n}(\mathbf{y}, a) \in I^{-}$if and only if

$$
\mathbf{y} \in\left\{\mathbf{b}_{n-2}^{-1}\left[\begin{array}{ccc}
A & \cdot & B \\
\dot{C} & a & \cdot \\
C & \cdot & D
\end{array}\right] \mathbf{b}_{n-2} \mid\left[\begin{array}{cc}
A & B \\
C & D
\end{array}\right] \in \mathrm{GO}_{n-3}^{-}(q), a=\operatorname{det}\left[\begin{array}{cc}
A & B \\
C & D
\end{array}\right]\right\} .
$$

Again, $a=a^{-1}= \pm 1$. Thus, $I^{-}=U_{n} L_{n}^{-}$, where $L_{n}^{-} \cong \operatorname{GO}_{n-3}^{-}(q)$. Furthermore,

$$
\left|L_{n}^{-}\right|=2 q^{(m-1)(m-2)}\left(q^{m-1}+1\right)\left(q^{2 m-4}-1\right)\left(q^{2 m-6}-1\right) \ldots\left(q^{2}-1\right) .
$$

Since the characters $\lambda^{0}, \lambda^{+}$and $\lambda^{-}$are non-trivial, the orders of their inertia subgroups are pairwise distinct and the sizes of the four orbits add up to $q^{n-2}$, the set $\left\{1_{U}, \lambda^{0}, \lambda^{+}, \lambda^{-}\right\}$is a set of representatives for the orbits of $L_{n}$ on $\operatorname{Irr}\left(U_{n}\right)$.

When $n=3$ the orbits of $\lambda^{0}, \lambda^{-}$do not exist and we have $L_{3}^{+}:=\mathrm{GO}_{0}^{+}(q)=\{1\}$ by definition. 


\subsection{Irreducible characters of $P_{n}$}

We still assume that $q$ is an arbitrary prime power and that $n=2 m+1 \geqslant 3$. For simplicity we set $P:=P_{n}, L:=L_{n}$ and $U:=U_{n}$. We obtain four types of irreducible characters of $P$ according to which character of $1_{U}, \lambda^{0}$ and $\lambda^{ \pm}$they cover:

Type 1: the characters with $U$ in their kernel,

Type 0: the characters covering $\lambda^{0}$,

Type + : the characters covering $\lambda^{+}$,

Type - : the characters covering $\lambda^{-}$.

Note that for $n=3$ there are no characters of Type 0 or Type - . For all odd $n \geqslant 3$ the characters of Type 1 are parameterized by the irreducible characters of $L$ via inflation and we write ${ }^{1} \psi_{\sigma}:=\operatorname{Infl}_{L}^{P} \sigma$ for $\sigma \in \operatorname{Irr}(L)$. We have ${ }^{1} \psi_{\sigma}(1)=\sigma(1)$.

Since $\lambda^{0}, \lambda^{ \pm}$are linear and $I^{0}=U \rtimes \tilde{P}_{n-2}, I^{ \pm}=U \rtimes L_{n}^{ \pm}$the characters $\lambda^{0}$ and $\lambda^{ \pm}$can be extended trivially to characters $\hat{\lambda}^{0} \in \operatorname{Irr}\left(I^{0}\right)$ and $\hat{\lambda}^{ \pm} \in \operatorname{Irr}\left(I^{ \pm}\right)$, respectively. We have a bijection between $\operatorname{Irr}\left(P_{n-2}\right)$ and $\operatorname{Irr}\left(\tilde{P}_{n-2}\right)$ sending $\mu \in \operatorname{Irr}\left(P_{n-2}\right)$ to the character $\tilde{\mu}:=\left(\mu \otimes 1_{A}\right) \downarrow_{\tilde{P}_{n-2}}^{P_{n-2} \times A}$. The irreducible characters of $P$ of Type 0 are parameterized by $\operatorname{Irr}\left(P_{n-2}\right)$ and the character of $P$ corresponding to $\mu \in \operatorname{Irr}\left(P_{n-2}\right)$ is ${ }^{0} \psi_{\mu}:=\left(\hat{\lambda}^{0} \cdot \operatorname{Infl}_{\tilde{P}_{n-2}}^{I^{0}} \tilde{\mu}\right) \uparrow_{I^{0}}^{P}$. We have ${ }^{0} \psi_{\mu}(1)=\left(q^{2 m-2}-1\right) \mu(1)$. The irreducible characters of $P$ of Type \pm are parameterized by $\operatorname{Irr}\left(\mathrm{GO}_{n-3}^{ \pm}(q)\right)$ and the character of $P$ corresponding to $\vartheta \in \operatorname{Irr}\left(\mathrm{GO}_{n-3}^{ \pm}(q)\right)$ is ${ }^{ \pm} \psi_{\vartheta}:=\left(\hat{\lambda}^{ \pm} \cdot \operatorname{Infl}_{L_{n}^{ \pm}}^{I^{ \pm}} \vartheta\right) \uparrow_{I^{ \pm}}$. For the degrees of these characters, we have ${ }^{ \pm} \psi_{\vartheta}(1)=\frac{1}{2} q^{m-1}\left(q^{m-1} \pm 1\right)(q-1) \vartheta(1)$.

Let $\varepsilon \in\{1,0,+,-\}$. In the same way as $[\mathbf{2}, \S 2]$, we use additive extension to expand the notation ${ }^{\varepsilon} \psi_{\sigma}$ to non-irreducible characters $\sigma$. For example, if $\sigma=\sum m_{j} \sigma_{j}$ with $\sigma_{j} \in \operatorname{Irr}(L)$, we set ${ }^{1} \psi_{\sigma}:=\sum m_{j}{ }^{1} \psi_{\sigma_{j}}$.

\section{Preliminary results on induced characters}

In this section we provide some information on certain characters of $P$ which are induced from various subgroups. These results will be used in subsequent sections when we study the restriction of Harish-Chandra induced characters via Mackey's theorem.

\subsection{Group theoretical lemmas}

We use the setting and notation from $\S \S 2$ and 3. In particular, we fix an odd integer $n=2 m+1$, where $m \geqslant 1$. We choose simple reflections $s_{1}, s_{2}, \ldots, s_{m}$ generating the Weyl group $W$ of $G_{n}$ and define elements $s, t \in W$ as in $\S 2.2$. The following results are similar to [2, Lemmas 3.1 and 3.2$]$.

Lemma 4.1. Assume $m \geqslant 2$. Let $J:=\left\{s_{1}, s_{2}, \ldots, s_{m-1}\right\}$ and $s, t \in G_{n}$ as in $\S 2.2$. We write $W_{J}$ for the subgroup of $W$ generated by $J$ and $D_{J, J}$ for the set of distinguished representatives for $W_{J} \backslash W / W_{J}$ as in $[\mathbf{3}, \S 2.7]$ and define $K:={ }^{s} J \cap J$. Then:

(a) $D_{J, J}=\{1, s, t\}$

(b) ${ }^{t} P \cap P=L$;

(c) $K=\left\{s_{1}, \ldots, s_{m-2}\right\}$ and ${ }^{s} P \cap P=\left({ }^{s} U \cap U\right)\left({ }^{s} L \cap U\right)\left({ }^{s} U \cap L\right) L_{K}$. Moreover, $Q_{K}:=$ $\left({ }^{s} U \cap L\right) L_{K}$ is the standard parabolic subgroup of $L=L_{J}$ corresponding to $K \subseteq J$ with Levi decomposition $Q_{K}=\left({ }^{s} U \cap L\right) \rtimes L_{K}$, where

$$
{ }^{s} U \cap L=\left\{\left[\begin{array}{lll}
1 & & \\
& \mathbf{u}_{n-2}(\mathbf{v}) & \\
& & 1
\end{array}\right] \mid \mathbf{v} \in \mathbb{F}_{q}^{n-4}\right\}=U_{n-2}
$$


and

$$
L_{K}=\left\{\left[\begin{array}{ccccc}
a & & & & \\
& b & & & \\
& & \mathbf{x} & & \\
& & b^{-1} & \\
& & & a^{-1}
\end{array}\right] \mid a, b \in \mathbb{F}_{q}^{\times}, \mathbf{x} \in \mathrm{SO}_{n-4}(q)\right\} .
$$

Thus,

$$
Q_{K}=\left\{\left[\begin{array}{ccccc}
a & & & & \\
& b & * & * & \\
& & \mathbf{x} & * & \\
& & & b^{-1} & \\
& & & & a^{-1}
\end{array}\right] \mid a, b \in \mathbb{F}_{q}^{\times}, \mathbf{x} \in \mathrm{SO}_{n-4}(q)\right\}=A \times P_{n-2} .
$$

Setting

$$
A_{n-2}:={ }^{s} A=\left\{\left[\begin{array}{ccccc}
1 & & & & \\
& b & & & \\
& & I_{n-4} & & \\
& & & b^{-1} & \\
& & & & 1
\end{array}\right] \mid b \in \mathbb{F}_{q}^{\times}\right\} \cong \mathbb{F}_{q}^{\times}
$$

and

$$
\tilde{L}_{n-2}^{\prime}:=\left\{\left[\begin{array}{ccc}
I_{2} & & \\
& \mathrm{x} & \\
& & I_{2}
\end{array}\right] \mid \mathrm{x} \in \mathrm{SO}_{n-4}(q)\right\} \cong \operatorname{SO}_{n-4}(q),
$$

we have $L_{K}=A \times A_{n-2} \times L_{n-2}^{\prime}$;

(d) we set $R:=\left({ }^{s} U \cap U\right)\left({ }^{s} L \cap U\right)$. We have:
(i) ${ }^{s} L \cap U=\left\{\mathbf{u}_{n}(\mathbf{v}) \mid \mathbf{v} \in \mathbb{F}_{q}^{n-2}, v_{m-1}=v_{m-1}^{\prime}=0\right\}$;
(ii) ${ }^{s} L \cap L=L_{K}$;
(iii) ${ }^{s} U \cap U=\left\{\mathbf{u}_{n}\left(\left[\begin{array}{llll}v_{m-1} & 0 & \ldots & 0\end{array}\right]^{\mathrm{tr}}\right) \mid v_{m-1} \in \mathbb{F}_{q}\right\},\left|{ }^{s} U \cap U\right|=q,{ }^{t} U \cap U=\{1\}$;
(iv) ${ }^{s} P \cap P=R Q_{K}$;
(v) $R=\left\{\mathbf{u}_{n}(\mathbf{v}) \mid \mathbf{v} \in \mathbb{F}_{q}^{n-2}, v_{m-1}^{\prime}=0\right\}$ and $[U: R]=q$;
(vi) $\left[P_{K}: R Q_{K}\right]=q$, where $P_{K}=U Q_{K}$ is the parabolic subgroup of $G=G_{n}$ corresponding to $K \subseteq\left\{s_{1}, \ldots, s_{m}\right\}$;
(vii) $Q_{K}=A \times P_{n-2}$.

Proof. (a) Follows from [2, Lemma 3.1(a)] since the Weyl groups of type $B_{m}$ and $C_{m}$ are canonically isomorphic.

(b) Follows from a straightforward calculation.

(c) It follows from (2.2) and (2.3) that $s$ commutes with $s_{1}, \ldots, s_{m-2}$ and that $s s_{m-1} s \notin J$. Thus, $K=\left\{s_{1}, \ldots, s_{m-2}\right\}$. Now [3, Theorem 2.8.7(a) and Proposition 2.8.9] imply the remaining statements in (c).

(d) follows from elementary matrix calculations.

To keep the notation simple we identify $G_{n-2 j}$ with $\operatorname{diag}\left(I_{j}, G_{n-2 j}, I_{j}\right) \leqslant G_{n}$, so that we can consider $G_{n-2 j}$ and its subgroups $U_{n-2 j}, L_{n-2 j}^{\prime}$ and so on as subgroups of $G_{n}$. For $m \geqslant 3$ we set $\tilde{P}_{n-4}:=U_{n-4} \tilde{L}_{n-4}$, where

$$
\tilde{L}_{n-4}:=\left\{\left[\begin{array}{ccc}
a I_{3} & & \\
& \mathbf{x} & \\
& & a^{-1} I_{3}
\end{array}\right] \mid \mathbf{x} \in \operatorname{SO}_{n-6}(q), a \in \mathbb{F}_{q}^{\times}\right\}
$$


and

$$
U_{n-4}=\left\{\left[\begin{array}{lll}
I_{2} & & \\
& \mathbf{u}_{n-4}(\mathbf{v}) & \\
& & I_{2}
\end{array}\right] \mid \mathbf{v} \in \mathbb{F}_{q}^{n-6}\right\} .
$$

Furthermore, we set $r:=s_{m-1}$,

$$
\begin{aligned}
& A_{n, n-2}:=\left\{\left[\begin{array}{lll}
a I_{2} & & \\
& I_{n-4} & \\
& & a^{-1} I_{2}
\end{array}\right] \mid a \in \mathbb{F}_{q}^{\times}\right\}, \\
& A_{n-4}={ }^{r} A_{n-2}=\left\{\left[\begin{array}{ccccc}
I_{2} & & & & \\
& a & & & \\
& & I_{n-6} & & \\
& & & a^{-1} & \\
& & & & I_{2}
\end{array}\right] \mid a \in \mathbb{F}_{q}^{\times}\right\}
\end{aligned}
$$

and additionally

$$
\begin{aligned}
L_{n-2}^{\prime} & =\left\{\left[\begin{array}{lll}
I_{2} & & \\
& \mathbf{x} & \\
& & I_{2}
\end{array}\right] \mid \mathbf{x} \in \mathrm{SO}_{n-4}(q)\right\}, \quad L_{n-4}^{\prime}=\left\{\left[\begin{array}{ccc}
I_{3} & & \\
& \mathbf{x} & \\
& & I_{3}
\end{array}\right] \mid \mathbf{x} \in \operatorname{SO}_{n-6}(q)\right\}, \\
Q_{K}^{\prime}:= & A \times U_{n-2} \tilde{P}_{n-4} \\
= & \left\{\left[\begin{array}{cccccc}
a & & & & & \\
& b & * & * & * & * \\
& b & * & * & * \\
& & \mathbf{x} & * & * \\
& & b^{-1} & * \\
& & & b^{-1} & \\
& & & & a^{-1}
\end{array}\right] \mid \mathbf{x} \in \operatorname{SO}_{n-6}(q), a, b \in \mathbb{F}_{q}^{\times}\right\} \leqslant Q_{K} .
\end{aligned}
$$

Lemma 4.2. Let $m \geqslant 3$. With the above notation, we have

$$
{ }^{r s}\left(R Q_{K}^{\prime}\right) \cap U \tilde{P}_{n-2}=\left({ }^{r} R\right) Y,
$$

where

$$
Y=\left({ }^{r} U_{n-2} \cap U_{n-2}\right)\left({ }^{r} L_{n-2} \cap U_{n-2}\right)\left({ }^{r} U_{n-2} \cap L_{n-2}\right)\left(A_{n, n-2} \times A_{n-4} \times L_{n-4}^{\prime}\right) .
$$

Furthermore, $A \times Y=A \times\left({ }^{r} P_{n-2} \cap P_{n-2}\right)$.

Proof. We have $\tilde{P}_{n-2}=A_{n, n-2} U_{n-2} L_{n-2}^{\prime}$ and $Q_{K}^{\prime}=A U_{n-2} \tilde{P}_{n-4}$. Furthermore,

$$
{ }^{s r} A_{n, n-2}=\left\{\left[\begin{array}{lllll}
1 & & & & \\
& a I_{2} & & & \\
& & I_{n-6} & & \\
& & & a^{-1} I_{2} & \\
& & & & 1
\end{array}\right] \mid a \in \mathbb{F}_{q}^{\times}\right\}
$$

and thus $A \tilde{P}_{n-4}=A\left({ }^{s r} A_{n, n-2}\right) U_{n-4} L_{n-4}^{\prime}$. Since $A$ centralizes $U_{n-2}$, we have

$$
Q_{K}^{\prime}=U_{n-2} A\left({ }^{s r} A_{n, n-2}\right) U_{n-4} L_{n-4}^{\prime}=A U_{n-2}\left({ }^{s r} A_{n, n-2}\right) U_{n-4} L_{n-4}^{\prime} .
$$

Using that $A$ centralizes $U_{n-2}$, that $s$ normalizes $R U_{n-2}$, that $A_{n-2}={ }^{s} A$, that $s$ centralizes $U_{n-4} L_{n-4}^{\prime}$ and that $A_{n-2}$ and ${ }^{r} A_{n, n-2}$ centralize each other and normalize $U_{n-2}$, we get 


$$
\begin{aligned}
{ }^{r s}\left(R Q_{K}^{\prime}\right) & ={ }^{r s}\left(R A U_{n-2}\left({ }^{s r} A_{n, n-2}\right) U_{n-4} L_{n-4}^{\prime}\right) \\
& ={ }^{r s}\left(R U_{n-2}\right)\left({ }^{r s} A\right) A_{n, n-2}{ }^{r s}\left(U_{n-4} L_{n-4}^{\prime}\right) \\
& ={ }^{r}\left(R U_{n-2}\right)\left({ }^{r} A_{n-2}\right){ }^{r}\left({ }^{r} A_{n, n-2}\right){ }^{r}\left(U_{n-4} L_{n-4}^{\prime}\right) \\
& =\left({ }^{r} R\right) A_{n, n-2}{ }^{r}\left(A_{n-2} U_{n-2} U_{n-4} L_{n-4}^{\prime}\right) .
\end{aligned}
$$

An elementary matrix calculation shows that

$$
{ }^{r} R, A_{n, n-2} \subseteq U \tilde{P}_{n-2} \quad \text { and } \quad{ }^{r}\left(A_{n-2} U_{n-2} U_{n-4} L_{n-4}^{\prime}\right) \subseteq L_{n}^{\prime} .
$$

Hence,

$$
\begin{aligned}
{ }^{r s}\left(R Q_{K}^{\prime}\right) \cap U \tilde{P}_{n-2} & =\left({ }^{r} R\right) A_{n, n-2}\left[{ }^{r}\left(A_{n-2} U_{n-2} U_{n-4} L_{n-4}^{\prime}\right) \cap L_{n}^{\prime} \cap U \tilde{P}_{n-2}\right] \\
& =\left({ }^{r} R\right) A_{n, n-2}\left[{ }^{r}\left(A_{n-2} U_{n-2} U_{n-4} L_{n-4}^{\prime}\right) \cap\left(L_{n}^{\prime} \cap U \tilde{P}_{n-2}\right)\right] \\
& =\left({ }^{r} R\right) A_{n, n-2}\left[{ }^{r}\left(A_{n-2} U_{n-2} U_{n-4} L_{n-4}^{\prime}\right) \cap U_{n-2} L_{n-2}^{\prime}\right] \\
& =\left({ }^{r} R\right) Y,
\end{aligned}
$$

where $Y:=A_{n, n-2}\left[U_{n-2} L_{n-2}^{\prime} \cap^{r}\left(A_{n-2} U_{n-2} U_{n-4} L_{n-4}^{\prime}\right)\right]$. By Lemma 4.1(c) applied to $G_{n-2}$, we have

$$
{ }^{r} P_{n-2} \cap P_{n-2}=\left({ }^{r} U_{n-2} \cap U_{n-2}\right)\left({ }^{r} L_{n-2} \cap U_{n-2}\right)\left({ }^{r} U_{n-2} \cap L_{n-2}\right)\left(A_{n-2} \times A_{n-4} \times L_{n-4}^{\prime}\right),
$$

where

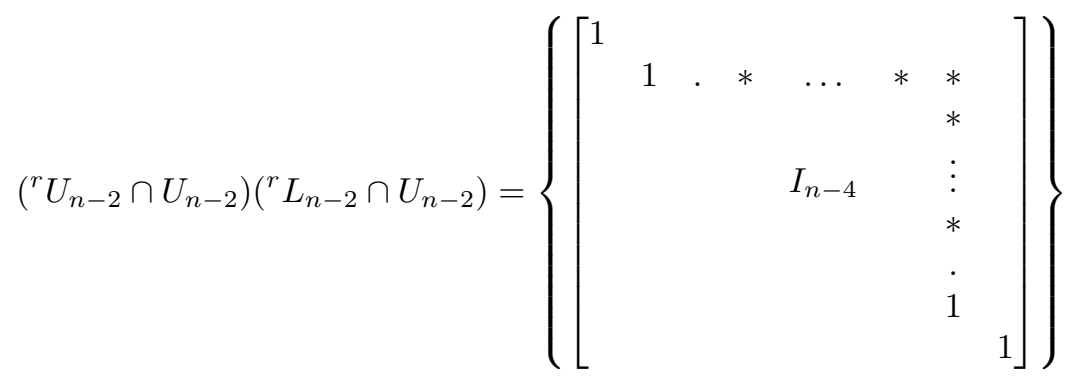

and

$$
\begin{aligned}
& \left({ }^{r} U_{n-2} \cap L_{n-2}\right)\left(A_{n-2} \times A_{n-4} \times L_{n-4}^{\prime}\right) \\
& \quad=\left\{\left[\begin{array}{cccccc}
1 & & & & \\
& a & & & & \\
& & b & * & * & \\
& & & \mathbf{x} & * & \\
& & & b^{-1} & & \\
& & & & a^{-1} & \\
& & & & & 1
\end{array}\right] \mid \mathbf{x} \in \operatorname{SO}_{n-6}(q), a, b \in \mathbb{F}_{q}^{\times}\right\} .
\end{aligned}
$$

We set

$$
\begin{aligned}
Y^{\prime} & :=\left({ }^{r} U_{n-2} \cap U_{n-2}\right)\left({ }^{r} L_{n-2} \cap U_{n-2}\right)\left({ }^{r} U_{n-2} \cap L_{n-2}\right)\left(A_{n-4} \times L_{n-4}^{\prime}\right) \\
& =\left\{\left[\begin{array}{ccccccc}
1 & & & & & & \\
& 1 & \cdot & * & * & * & \\
& & b & * & * & * \\
& & & \mathbf{x} & * & * & \\
& & & & b^{-1} & \cdot & \\
& & & & & 1 & \\
& & & & &
\end{array}\right] \mid \mathbf{x} \in \operatorname{SO}_{n-6}(q), b \in \mathbb{F}_{q}^{\times}\right\},
\end{aligned}
$$


so that ${ }^{r} P_{n-2} \cap P_{n-2}=A_{n-2} Y^{\prime}$. We can see from (4.6) that $Y^{\prime} \subseteq U_{n-2} L_{n-2}^{\prime}$. An elementary matrix calculation shows that ${ }^{r} L_{n-2} \cap U_{n-2}={ }^{r} U_{n-4}$. Using that $r$ centralizes $L_{n-4}^{\prime}$, that $U_{n-4}$ normalizes $U_{n-2}$ and that $A_{n-2}$ normalizes the subgroup $U_{n-2}$ and centralizes $U_{n-4}$, we get

$$
\begin{aligned}
Y^{\prime} & \subseteq{ }^{r} U_{n-2}{ }^{r} U_{n-4}{ }^{r} U_{n-2}{ }^{r} A_{n-2}{ }^{r} L_{n-4}^{\prime}={ }^{r}\left(U_{n-2} U_{n-4} U_{n-2} A_{n-2} L_{n-4}^{\prime}\right) \\
& ={ }^{r}\left(A_{n-2} U_{n-2} U_{n-4} L_{n-4}^{\prime}\right)
\end{aligned}
$$

and thus

$$
Y^{\prime} \leqslant U_{n-2} L_{n-2}^{\prime} \cap^{r}\left(A_{n-2} U_{n-2} U_{n-4} L_{n-4}^{\prime}\right) .
$$

Because $P_{n-2}^{\prime}:=U_{n-2} L_{n-2}^{\prime} \subseteq U_{n-2} L_{n-2}=P_{n-2}$, we get

$$
\begin{aligned}
U_{n-2} L_{n-2}^{\prime} \cap{ }^{r}\left(A_{n-2} U_{n-2} U_{n-4} L_{n-4}^{\prime}\right) & \leqslant P_{n-2}^{\prime} \cap{ }^{r} P_{n-2} \\
& =\left(P_{n-2}^{\prime} \cap P_{n-2}\right) \cap{ }^{r} P_{n-2} \\
& =P_{n-2}^{\prime} \cap\left({ }^{r} P_{n-2} \cap P_{n-2}\right) \\
& =P_{n-2}^{\prime} \cap A_{n-2} Y^{\prime}=Y^{\prime} .
\end{aligned}
$$

Hence, $Y^{\prime}=U_{n-2} L_{n-2}^{\prime} \cap{ }^{r}\left(A_{n-2} U_{n-2} U_{n-4} L_{n-4}^{\prime}\right)$ and $Y=A_{n, n-2} Y^{\prime}$. Now the claim follows.

\subsection{Restriction of Harish-Chandra induced characters}

Let $m \geqslant 1$ and let $\sigma$ be an irreducible character of $L$ with $A \leqslant \operatorname{ker}(\sigma)$. In this section we study $R_{L}^{G}(\sigma):=\left(\operatorname{Infl}_{L}^{P} \sigma\right) \uparrow_{P}^{G}$, the character of $G$ which is obtained from $\sigma$ by Harish-Chandra induction. We proceed along the lines of $[2, \S 3]$. Suppressing notation for inflation, Mackey's theorem and Lemma 4.1 give us

$$
\begin{aligned}
R_{L}^{G}(\sigma) \downarrow_{P}^{G} & =\sigma+{ }^{s} \sigma \downarrow_{\downarrow_{s} P_{P \cap P} \uparrow^{P} s_{s} P \cap P}+{ }^{t} \sigma \downarrow_{t}^{t} P \cap P \uparrow^{t}{ }_{t}^{P} P \cap P \\
& =\sigma+{ }^{s} \sigma \downarrow_{R Q_{K}}^{s}{ }_{R}{ }_{R Q_{K}}+{ }^{t} \sigma \downarrow_{L}^{t} P{ }_{L} P .
\end{aligned}
$$

Since $R Q_{K}={ }^{s} P \cap P$ is $s$-invariant, we have ${ }^{s} \sigma \downarrow_{R Q_{K}}^{s}={ }^{s} \sigma \downarrow^{s} P Q_{K}={ }^{s}\left(\sigma \downarrow_{R Q_{K}}^{P}\right)$. And, furthermore, $\sigma \downarrow_{R Q_{K}}^{P}=\operatorname{Infl}_{P_{n-2}}^{R Q_{K}}\left(\sigma \downarrow_{P_{n-2}}^{L^{\prime}}\right)$. Hence, ${ }^{s} \sigma \downarrow^{s} P Q_{K} \uparrow_{R Q_{K}}$ is a sum of characters of the form $\left({ }^{s} \nu\right) \uparrow_{R Q_{K}}^{P}$, where $\nu \in \operatorname{Irr}\left(P_{n-2}\right)$ is considered as a character of $R Q_{K}=R\left(A \times P_{n-2}\right)$ via inflation. Thus, we have to determine $\left({ }^{s} \nu\right) \uparrow P_{R Q_{K}}$ for $\nu \in \operatorname{Irr}\left(P_{n-2}\right)$ of Type $1,0, \pm$. In parts (d) and (e) of the following theorem we need subgroups $P_{n-3}^{ \pm}$of $L_{n}^{ \pm}$, which are defined as follows: we set

$$
P_{n-3}^{+}:=\left\{\left[\begin{array}{ccccccc}
a & & & & & & \\
& b & * & \cdot & * & * & \\
& & A & \cdot & B & * & \\
& & \cdot & a & . & \cdot & \\
& C & \cdot & D & * & \\
& & & & b^{-1} & \\
& & & & & a
\end{array}\right] \mid\left[\begin{array}{cc}
A & B \\
C & D
\end{array}\right] \in \mathrm{GO}_{n-5}^{+}(q), a=\operatorname{det}\left[\begin{array}{cc}
A & B \\
C & D
\end{array}\right], b \in \mathbb{F}_{q}^{\times}\right\}
$$

for $m>2$ and

$$
P_{2}^{+}:=\left\{\operatorname{diag}\left(1, b, 1, b^{-1}, 1\right) \mid b \in \mathbb{F}_{q}^{\times}\right\} \cong \mathbb{F}_{q}^{\times}
$$


for $m=2$. For $m>2$ we define $P_{n-3}^{-}$to be the subgroup of $L_{n}^{-}$consisting of all matrices $\mathbf{s}_{n}(\mathbf{y}, a)$ with

$$
\mathbf{y} \in\left\{\mathbf{b}_{n-2}^{-1}\left[\begin{array}{ccccc}
b & * & \cdot & * & * \\
& A & \cdot & B & * \\
& \cdot & a & \cdot & \cdot \\
C & \cdot & D & * \\
& & & & b^{-1}
\end{array}\right] \mathbf{b}_{n-2} \mid\left[\begin{array}{cc}
A & B \\
C & D
\end{array}\right] \in \mathrm{GO}_{n-5}^{-}(q), a=\operatorname{det}\left[\begin{array}{cc}
A & B \\
C & D
\end{array}\right], b \in \mathbb{F}_{q}^{\times}\right\},
$$

where the matrix $\mathbf{b}_{n-2}$ is defined in $\S 3.2$. The following theorem and parts of its proof are analogous to [2, Theorem 3.3].

Theorem 4.3. Assume $m \geqslant 2$. Let $\sigma \in \operatorname{Irr}(L)$ with $A \leqslant \operatorname{ker}(\sigma)$. Then the following statements hold.

(a) ${ }^{t} \sigma \downarrow_{L}^{t} P={ }^{t} \sigma$. In particular, ${ }^{t} \sigma \downarrow_{t}^{t} P \cap P \uparrow_{t}^{P} P \cap P=\left({ }^{t} \sigma\right) \uparrow_{L}^{P}$.

(b) Let $\nu \in \operatorname{Irr}\left(P_{n-2}\right)$ be of Type 1. We view $\nu$ as an irreducible character of the groups $L_{K}=A \times L_{n-2}=A \times A_{n-2} \times L_{n-2}^{\prime}$ and $R Q_{K}$ via inflation. Then

$$
\left({ }^{s} \nu\right) \uparrow_{R Q_{K}}^{P}={ }^{0} \psi_{\nu}+{ }^{1} \psi_{\Sigma},
$$

where $\Sigma:=R_{L_{K}}^{L}\left({ }^{s} \nu\right)$.

(c) Suppose $m \geqslant 3$. Let $r:=s_{m-1}$ and let $\nu \in \operatorname{Irr}\left(P_{n-2}\right)$ be of Type 0 , that is, there is $\nu_{0} \in \operatorname{Irr}\left(P_{n-4}\right)$ such that $\nu={ }^{0} \psi_{\nu_{0}}$. By inflation, we consider $\nu_{0}$ as an irreducible character of

$$
{ }^{r} P_{n-2} \cap P_{n-2}=\left\{\left[\begin{array}{ccccccc}
1 & & & & & & \\
& a & & * & * & * & \\
& & b & * & * & * & \\
& & & \mathbf{x} & * & * & \\
& & & & b^{-1} & \cdot & \\
& & & & & a^{-1} & \\
& & & & & &
\end{array}\right] \mid \mathbf{x} \in \mathrm{SO}_{n-6}(q), a, b \in \mathbb{F}_{q}^{\times}\right\} .
$$

Since ${ }^{r} P_{n-2} \cap P_{n-2}$ is $r$-invariant, we have ${ }^{r} \nu_{0} \in \operatorname{Irr}\left({ }^{r} P_{n-2} \cap P_{n-2}\right)$. Then

$$
\left({ }^{s} \nu\right) \uparrow_{R Q_{K}}^{P}={ }^{0} \psi_{\Sigma},
$$

where $\Sigma:=\left({ }^{r} \nu_{0}\right) \uparrow_{r}^{P_{n-2}} P_{n-2} \cap P_{n-2}$.

(d) Let $\nu \in \operatorname{Irr}\left(P_{n-2}\right)$ be of Type + , say $\nu={ }^{+} \psi_{\vartheta_{0}}$ for some $\vartheta_{0} \in \operatorname{Irr}\left(L_{n-2}^{+}\right)$, and let $\vartheta:=R_{L_{n-2}^{+}}^{L_{n}^{+}}\left(\vartheta_{0}\right):=\tilde{\vartheta}_{0} \uparrow_{P_{n-3}^{+}}^{L_{n}^{+}}$, where $\tilde{\vartheta}_{0} \in \operatorname{Irr}\left(P_{n-3}^{+}\right)$is the inflation of $\vartheta_{0}$ given by

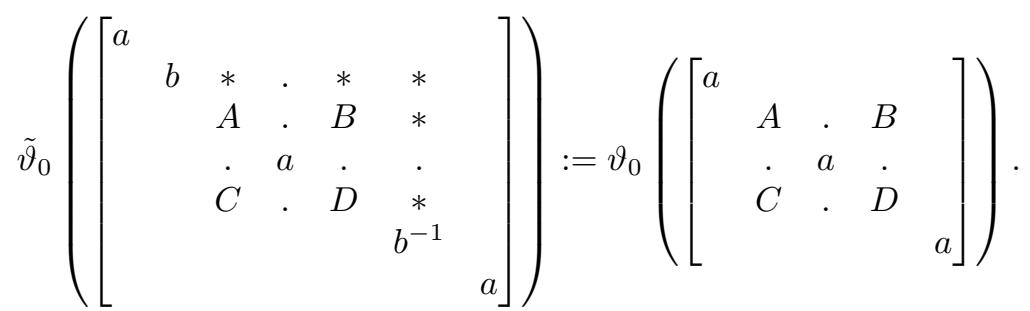

Then $\left({ }^{s} \nu\right) \uparrow_{R Q_{K}}^{P}={ }^{+} \psi_{\vartheta}$. 
(e) Let $\nu \in \operatorname{Irr}\left(P_{n-2}\right)$ be of Type -, say $\nu={ }^{-} \psi_{\vartheta_{0}}$ for some $\vartheta_{0} \in \operatorname{Irr}\left(L_{n-2}^{-}\right)$, and let $\vartheta:=R_{L_{n-2}^{-}}^{L_{n}^{-}}\left(\vartheta_{0}\right):=\tilde{\vartheta}_{0} \uparrow_{P_{n-3}^{-}}^{L_{n}^{-}}$, where $\tilde{\vartheta}_{0} \in \operatorname{Irr}\left(P_{n-3}^{-}\right)$is the inflation of $\vartheta_{0}$ given by

$$
\begin{gathered}
\tilde{\vartheta}_{0}\left(\mathbf{s}_{n}\left(\mathbf{b}_{n-2}^{-1}\left[\begin{array}{ccccc}
b & * & \cdot & * & * \\
& A & \cdot & B & * \\
& \cdot & a & \cdot & \cdot \\
C & \cdot & D & * \\
& & & b^{-1}
\end{array}\right] \mathbf{b}_{n-2}, a\right)\right) \\
:=\vartheta_{0}\left(\mathbf{s}_{n-2}\left(\mathbf{b}_{n-4}^{-1}\left[\begin{array}{ccc}
A & \cdot & B \\
\dot{C} & a & \cdot \\
C & \cdot & D
\end{array}\right] \mathbf{b}_{n-4}, a\right)\right) .
\end{gathered}
$$

Then $\left({ }^{s} \nu\right) \uparrow_{R Q_{K}}^{P}={ }^{-} \psi_{\vartheta}$.

Proof. (a) A straightforward computation shows that $t$ normalizes $L^{\prime}$ and $L$. It follows that ${ }^{t} \sigma \downarrow_{L}^{t} P={ }^{t} \sigma$, proving (a).

(b) Note that $s$ normalizes $L_{K}$, so that ${ }^{s} \nu \in \operatorname{Irr}\left(L_{K}\right)$. We first consider the special case that $A_{n-2} \leqslant \operatorname{ker}(\nu)$. Then ${ }^{s} \nu=\nu$ since $s$ centralizes $L_{n-2}^{\prime}$. Let $\tilde{\nu}$ be the irreducible character of $\tilde{P}_{n-2}$ corresponding to $\nu$ as in $\S 3.3$. We also write $\tilde{\nu}$ for the inflation of $\tilde{\nu}$ to $U \tilde{P}_{n-2}$. By Mackey's theorem, we have

$$
\begin{aligned}
\left\langle\left(\hat{\lambda}^{0} \cdot \tilde{\nu}\right) \uparrow_{U \tilde{P}_{n-2}}^{U Q_{K}}, \nu \uparrow_{R Q_{K}}^{U Q_{K}}\right\rangle_{U Q_{K}} & =\left\langle\hat{\lambda}^{0} \cdot \tilde{\nu}, \nu \uparrow_{R Q_{K} \downarrow}^{U Q_{K}} \downarrow_{U \tilde{P}_{n-2}}^{U Q_{K}}\right\rangle_{U \tilde{P}_{n-2}} \\
& \geqslant\left\langle\hat{\lambda}^{0} \cdot \tilde{\nu}, \nu \downarrow_{R \tilde{P}_{n-2}}^{R Q_{K}} \uparrow_{R \tilde{P}_{n-2}}\right\rangle_{U \tilde{P}_{n-2}} \\
& =\left\langle\hat{\lambda}^{0} \downarrow_{R \tilde{P}_{n-2}}^{U \tilde{P}_{n-2}} \cdot \tilde{\nu}, \tilde{\nu}\right\rangle_{R \tilde{P}_{n-2}} \\
& =\langle\tilde{\nu}, \tilde{\nu}\rangle_{R \tilde{P}_{n-2}}=1
\end{aligned}
$$

since $R \leqslant \operatorname{ker}\left(\hat{\lambda}^{0}\right)$ and $\hat{\lambda}^{0} \downarrow_{\tilde{P}_{n-2}}^{U \tilde{P}_{n-2}}=1_{\tilde{P}_{n-2}}$. It follows that ${ }^{0} \psi_{\nu}=\left(\hat{\lambda}^{0} \cdot \tilde{\nu}\right) \uparrow_{U \tilde{P}_{n-2}}^{P}$ is an irreducible constituent of $\nu \uparrow P_{R Q_{K}}=\left({ }^{s} \nu\right) \uparrow_{R Q_{K}}$.

Furthermore, $\nu$ is a constituent of $\nu \uparrow_{R Q_{K}}^{U Q_{K}}$. Hence, ${ }^{1} \psi_{\Sigma}=\left({ }^{s} \nu\right) \uparrow_{U Q_{K}}^{P}=\nu \uparrow_{U Q_{K}}^{P}$ is a subcharacter of $\nu \uparrow_{R Q_{K}}^{P}=\left({ }^{s} \nu\right) \uparrow_{R Q_{K}}^{P}$. Hence, $\left({ }^{s} \nu\right) \uparrow_{R Q_{K}}^{P}={ }^{0} \psi_{\nu}+{ }^{1} \psi_{\Sigma}+(\ldots)$. Comparing degrees, we get $\left({ }^{s} \nu\right) \uparrow_{R Q_{K}}^{P}={ }^{0} \psi_{\nu}+{ }^{1} \psi_{\Sigma}$.

Now we deal with the general case. Write $\nu=1_{A} \otimes \zeta \otimes \nu^{\prime}$, where $\zeta \in \operatorname{Irr}\left(A_{n-2}\right)$ and $\nu^{\prime} \in \operatorname{Irr}\left(L_{n-2}^{\prime}\right)$. Then ${ }^{s} \nu={ }^{s} \zeta \otimes 1_{A_{n-2}} \otimes \nu^{\prime}$. Considering ${ }^{s} \zeta$ as a linear character of $P$, via inflation we obtain $\left({ }^{s} \nu\right) \uparrow_{R Q_{K}}^{P}={ }^{s} \zeta \cdot\left({ }^{0} \psi_{\nu^{\prime}}+{ }^{1} \psi_{\Sigma^{\prime}}\right)$, where $\Sigma^{\prime}:=R_{L_{K}}^{L}\left(\nu^{\prime}\right)$. Hence, we get

$$
\left({ }^{s} \nu\right) \uparrow P_{R Q_{K}}={ }^{s} \zeta \cdot{ }^{0} \psi_{\nu^{\prime}}+{ }^{s} \zeta \cdot{ }^{1} \psi_{\Sigma^{\prime}}={ }^{0} \psi_{\nu}+{ }^{1} \psi_{\Sigma} .
$$

(c) By assumption, $\nu \in \operatorname{Irr}\left(P_{n-2}\right)$ is of Type 0 . Let $\lambda_{n-2}^{0}$ be the irreducible character of $U_{n-2}$ analogous to $\lambda^{0} \in \operatorname{Irr}(U)$ and let $\hat{\lambda}_{n-2}^{0}$ be the extension of $\lambda_{n-2}^{0}$ to $U_{n-2} \tilde{P}_{n-4}$ such that $\hat{\lambda}_{n-2}^{0} \downarrow_{\tilde{P}_{n-4}}^{U_{n-2} \tilde{P}_{n-4}}=1_{\tilde{P}_{n-4}}$. By definition,

$$
\nu=\left(\hat{\lambda}_{n-2}^{0} \cdot \operatorname{Infl}_{\tilde{P}_{n-4}}^{U_{n-2} \tilde{P}_{n-4}}\left(\tilde{\nu}_{0}\right)\right) \uparrow_{U_{n-2} \tilde{P}_{n-4}}^{P_{n-2}} .
$$

As before, we set $Q_{K}^{\prime}:=A \times U_{n-2} \tilde{P}_{n-4} \leqslant Q_{K}$. We inflate the characters in (4.9) over the normal subgroup $R A$ to $R Q_{K}^{\prime}$ and $R Q_{K}$, respectively. Suppressing the symbols for inflation, we obtain

$$
\nu=\left(\hat{\lambda}_{n-2}^{0} \cdot \tilde{\nu}_{0}\right) \uparrow_{R Q_{K}^{\prime}}^{R Q_{K}}
$$


Since $r \in P$ and $R Q_{K}$ is $s$-invariant, we have

$$
\begin{aligned}
\left({ }^{s} \nu\right) \uparrow_{R Q_{K}}^{P} & ={ }^{r}\left(\left({ }^{s} \nu\right) \uparrow_{R Q_{K}}^{P}\right) \\
& ={ }^{r}\left(\left({ }^{s}\left(\left(\hat{\lambda}_{n-2}^{0} \cdot \tilde{\nu}_{0}\right) \uparrow_{R Q_{K}^{\prime}}^{R Q_{K}}\right)\right) \uparrow_{R Q_{K}}^{P}\right) \\
& ={ }^{r}\left(\left({ }^{s} \hat{\lambda}_{n-2}^{0} \cdot{ }^{s} \tilde{\nu}_{0}\right) \uparrow_{s}^{R Q_{K}}\left(R Q_{K}^{\prime}\right) \uparrow_{R Q_{K}}^{P}\right) \\
& ={ }^{r}\left(\left({ }^{s} \hat{\lambda}_{n-2}^{0} \cdot{ }^{s} \tilde{\nu}_{0}\right) \uparrow_{s}^{P}\left(R Q_{K}^{\prime}\right)\right. \\
& =\left({ }^{s} \hat{\lambda}_{n-2}^{0} \cdot{ }^{r s} \tilde{\nu}_{0}\right) \uparrow_{r s}^{P}\left(R Q_{K}^{\prime}\right) .
\end{aligned}
$$

We set $\widetilde{\Sigma}:=\left(1_{A} \otimes \Sigma\right) \downarrow_{\tilde{P}_{n-2}}^{A \times P_{n-2}}$, so that ${ }^{0} \psi_{\Sigma}=\left(\hat{\lambda}^{0} \cdot \operatorname{Infl}_{\tilde{P}_{n-2}}^{\left.U \tilde{P}_{n-2}(\widetilde{\Sigma})\right) \uparrow_{U \tilde{P}_{n-2}}^{P}}\right.$.

By Lemma 4.2, we have

$$
U \tilde{P}_{n-2} \cap{ }^{r s}\left(R Q_{K}^{\prime}\right)=\left({ }^{r} R\right) Y
$$

with $Y \leqslant \tilde{P}_{n-2}$ and $A \times Y=A \times\left({ }^{r} P_{n-2} \cap P_{n-2}\right)$. Now

$$
{ }^{r s} U_{n-2}=\left\{\left[\begin{array}{ccccccccc}
1 & * & \cdot & * & * & * & \cdot & * & * \\
& 1 & \cdot & \cdot & \cdot & \cdot & \cdot & \cdot & * \\
& & 1 & \cdot & \cdot & \cdot & \cdot & \cdot & \cdot \\
& & & & & & \cdot & \cdot & * \\
& & & & I_{n-6} & & \cdot & \cdot & * \\
& & & & & \cdot & \cdot & * \\
& & & & & 1 & \cdot & \cdot \\
& & & & & & 1 & * \\
& & & & & & & 1
\end{array}\right]\right\} \leqslant U .
$$

Let $v=\left[v_{m-2}, \ldots, v_{1}, v_{0}, v_{1}^{\prime}, \ldots, v_{m-2}^{\prime}\right]^{\operatorname{tr}} \in \mathbb{F}_{q}^{n-4}$. Then

$$
{ }^{r s} \mathbf{u}_{n-2}(v)=\mathbf{u}_{n}\left(\left[*, 0, v_{m-2}^{\prime}\right]^{\operatorname{tr}}\right)
$$

and thus ${ }^{r s} \hat{\lambda}_{n-2}^{0}\left({ }^{r s} \mathbf{u}_{n-2}(v)\right)=\hat{\lambda}_{n-2}^{0}\left(\mathbf{u}_{n-2}(v)\right)=\xi\left(v_{m-2}^{\prime}\right)=\lambda^{0}\left({ }^{r s} \mathbf{u}_{n-2}(v)\right)$. Hence, the characters ${ }^{r s} \hat{\lambda}_{n-2}^{0}$ and $\lambda^{0}$ coincide on ${ }^{r s} U_{n-2}$. As above, we consider $\hat{\lambda}_{n-2}^{0} \cdot \tilde{\nu}_{0}$ and $\tilde{\nu}_{0}$ as irreducible characters of $R Q_{K}^{\prime}$ via inflation. Thus, ${ }^{r s} \hat{\lambda}_{n-2}^{0} \cdot{ }^{r s} \tilde{\nu}_{0} \in \operatorname{Irr}\left({ }^{r s}\left(R Q_{K}^{\prime}\right)\right)$. By restriction via (4.11), we can view ${ }^{r s} \hat{\lambda}_{n-2}^{0} \cdot{ }^{r s} \tilde{\nu}_{0}$ as a character of $\left({ }^{r} R\right) Y \leqslant U Y$. An elementary matrix calculation shows that ${ }^{s} R \subseteq R U_{n-2} \subseteq \operatorname{ker}\left(\tilde{\nu}_{0}\right)$. It follows that

$$
{ }^{r} R=\left\{\left[\begin{array}{ccccccccc}
1 & * & . & * & * & * & * & * & * \\
& 1 & \cdot & \cdot & \cdot & \cdot & \cdot & \cdot & * \\
& & 1 & \cdot & \cdot & \cdot & \cdot & \cdot & * \\
& & & & & & \cdot & \cdot & * \\
& & & & I_{n-6} & & \cdot & \cdot & * \\
& & & & & & \cdot & \cdot & * \\
& & & & & & & \cdot & \cdot \\
& & & & & & 1 & * \\
& & & & & & & 1
\end{array}\right]\right\} \operatorname{ker}\left({ }^{r s} \tilde{\nu}_{0}\right) .
$$

Since $Y$ normalizes ${ }^{r} R$ and $U$, the character ${ }^{r s} \tilde{\nu}_{0}$ has a unique extension to $U Y$ with $U$ in its kernel and we denote this extension also by ${ }^{r s} \tilde{\nu}_{0}$. An elementary matrix calculation 
shows that

$$
\left\{\left[\begin{array}{ccccccccc}
1 & \cdot & \cdot & \cdot & \cdot & \cdot & * & \cdot & * \\
& 1 & \cdot & \cdot & \cdot & \cdot & \cdot & \cdot & \cdot \\
& & 1 & \cdot & \cdot & \cdot & \cdot & \cdot & * \\
& & & & & & \cdot & \cdot & \cdot \\
& & & & I_{n-6} & & \cdot & \cdot & \cdot \\
& & & & & \cdot & \cdot & \cdot \\
& & & & & 1 & \cdot & \cdot \\
& & & & & & 1 & \cdot \\
& & & & & & & 1
\end{array}\right\}^{r s}\right\} \leqslant \operatorname{ker}\left(\hat{\lambda}_{n-2}^{0}\right)
$$

and it follows that ${ }^{r s} \hat{\lambda}_{n-2}^{0}$ coincides with $\lambda^{0}$ and $\hat{\lambda}^{0}$ on ${ }^{r} R$. Similarly, we see that $Y^{r s} \leqslant$ $\operatorname{ker}\left(\hat{\lambda}_{n-2}^{0}\right)$; hence, $Y \leqslant \operatorname{ker}\left({ }^{r s} \hat{\lambda}_{n-2}^{0}\right), \operatorname{ker}\left(\hat{\lambda}^{0}\right)$. Thus, ${ }^{r s} \hat{\lambda}_{n-2}^{0}$ coincides with $\hat{\lambda}^{0}$ on $Y$, too. Therefore, $\hat{\lambda}^{0}$ considered as an irreducible character of $U Y$ via restriction is an extension of ${ }^{r s} \hat{\lambda}_{n-2}^{0}$ to $U Y$ and $\hat{\lambda}^{0} \cdot{ }^{r s} \tilde{\nu}_{0}$ is an extension of ${ }^{r s} \hat{\lambda}_{n-2}^{0} \cdot{ }^{r s} \tilde{\nu}_{0}$ from $\left({ }^{r} R\right) Y$ to $U Y$. Thus, $\hat{\lambda}^{0} \cdot{ }^{r s} \tilde{\nu}_{0}$ is a subcharacter of $\left({ }^{r s} \hat{\lambda}_{n-2}^{0} \cdot{ }^{r s} \tilde{\nu}_{0}\right) \uparrow{ }_{(r R) Y}^{U Y}$ and therefore $\left(\hat{\lambda}^{0} \cdot{ }^{r s} \tilde{\nu}_{0}\right) \uparrow_{U Y}^{U \tilde{P}_{n-2}}$ is a subcharacter of $\left({ }^{r s} \hat{\lambda}_{n-2}^{0} \cdot{ }^{r s} \tilde{\nu}_{0}\right) \uparrow\left(\begin{array}{l}U \tilde{P}_{n-2} \\ \tilde{r}_{R} Y\end{array}\right.$. The isomorphism

$$
\tilde{P}_{n-2} \rightarrow P_{n-2},\left[\begin{array}{ccccc}
a & & & & \\
& a & * & * & \\
& & x & * & \\
& & & a^{-1} & \\
& & & & a^{-1}
\end{array}\right] \mapsto\left[\begin{array}{ccccc}
1 & & & & \\
& a & * & * & \\
& & x & * & \\
& & & a^{-1} & \\
& & & & 1
\end{array}\right]
$$

maps $Y$ onto the group ${ }^{r} P_{n-2} \cap P_{n-2}$ and hence induces bijections $\operatorname{Irr}\left(P_{n-2}\right) \rightarrow$ $\operatorname{Irr}\left(\tilde{P}_{n-2}\right)$ and $\operatorname{Irr}\left({ }^{r} P_{n-2} \cap P_{n-2}\right) \rightarrow \operatorname{Irr}(Y)$, which we both denote by $\phi$. We extend $\phi$ additively to non-irreducible characters of these groups. An elementary matrix calculation shows that $\widetilde{\Sigma}=\phi(\Sigma)$ and ${ }^{r s} \tilde{\nu}_{0}=\phi\left({ }^{r} \nu_{0}\right)$. The functoriality of induction implies that $\phi$ commutes with induction; hence,

$$
\widetilde{\Sigma}=\phi(\Sigma)=\phi\left(\left({ }^{r} \nu_{0}\right) \uparrow_{r}^{P_{n-2}} P_{n-2} \cap P_{n-2}\right)=\phi\left({ }^{r} \nu_{0}\right) \uparrow_{Y}^{\tilde{P}_{n-2}}=\left({ }^{r s} \tilde{\nu}_{0}\right) \uparrow_{Y}^{\tilde{P}_{n-2}}
$$

Again suppressing the notation for inflation, we get that

$$
\hat{\lambda}^{0} \cdot \widetilde{\Sigma}=\hat{\lambda}^{0} \cdot\left({ }^{r s} \tilde{\nu}_{0}\right) \uparrow_{U Y}^{U \tilde{P}_{n-2}}=\left(\hat{\lambda}^{0} \cdot{ }^{r s} \tilde{\nu}_{0}\right) \uparrow_{U Y}^{U \tilde{P}_{n-2}}
$$

is a subcharacter of

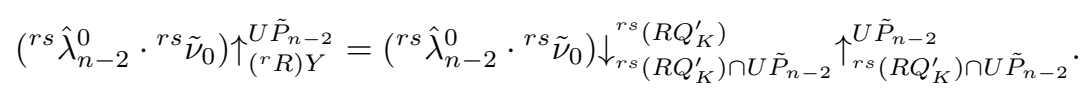

It follows from Mackey's theorem and (4.10) that $\hat{\lambda}^{0} \cdot \widetilde{\Sigma}$ is a subcharacter of

$$
\left({ }^{r s} \hat{\lambda}_{n-2}^{0} \cdot{ }^{r s} \tilde{\nu}_{0}\right) \uparrow_{r s}^{P}\left(R Q_{K}^{\prime}\right) \downarrow_{U}^{P} \tilde{P}_{n-2}=\left({ }^{s} \nu\right) \uparrow_{R Q_{K}}^{P} \downarrow_{U}^{P} \tilde{P}_{n-2} .
$$

Write $\hat{\lambda}^{0} \cdot \widetilde{\Sigma}=\sum_{i} m_{i} \chi_{i}$ with $\chi_{i} \in \operatorname{Irr}\left(U \tilde{P}_{n-2}\right)$; hence,

$$
\left({ }^{s} \nu\right) \uparrow_{R Q_{K}}^{P} \downarrow_{U \tilde{P}_{n-2}}^{P}=\sum_{i} m_{i} \chi_{i}+(\ldots)
$$

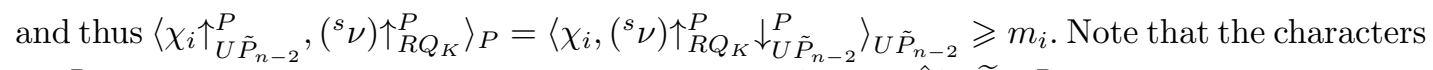
$\chi_{i} \uparrow_{U \tilde{P}_{n-2}}^{P}$ are irreducible by Clifford theory. Hence, ${ }^{0} \psi_{\Sigma}=\left(\hat{\lambda}^{0} \cdot \widetilde{\Sigma}\right) \uparrow_{U \tilde{P}_{n-2}}^{P}$ is a subcharacter of $\left({ }^{s} \nu\right) \uparrow_{R Q_{K}}^{P}$. We have 


$$
\begin{aligned}
\left({ }^{s} \nu\right) \uparrow_{R Q_{K}}^{P}(1) & =\left[P: R Q_{K}\right] \cdot \nu(1)=\frac{|U||L|}{|R|\left|Q_{K}\right|}\left(q^{n-5}-1\right) \nu_{0}(1) \\
& =q \cdot \frac{(q-1) \cdot\left|\mathrm{SO}_{n-2}(q)\right|}{(q-1) \cdot\left|P_{n-2}\right|}\left(q^{2 m-4}-1\right) \nu_{0}(1) \\
& =q \cdot \frac{\left(q^{2 m-2}-1\right)\left(q^{2 m-4}-1\right)}{q-1} \cdot \nu_{0}(1)
\end{aligned}
$$

and

$$
\begin{aligned}
{ }^{0} \psi_{\Sigma}(1) & =\left(q^{n-3}-1\right) \cdot \Sigma(1)=\left(q^{n-3}-1\right) \cdot\left[P_{n-2}:{ }^{r} P_{n-2} \cap P_{n-2}\right] \cdot \nu_{0}(1) \\
& =\left(q^{n-3}-1\right) \cdot \frac{\left|P_{n-2}\right|}{q^{n-5} \cdot(q-1) \cdot\left|P_{n-4}\right|} \cdot \nu_{0}(1) \\
& =q \cdot \frac{\left(q^{2 m-2}-1\right)\left(q^{2 m-4}-1\right)}{q-1} \cdot \nu_{0}(1) ;
\end{aligned}
$$

thus, $\left({ }^{s} \nu\right) \uparrow_{R Q_{K}}^{P}={ }^{0} \psi_{\Sigma}$, proving (c).

(d) We denote the inflation of the character $\vartheta_{0} \cdot \hat{\lambda}_{n-2}^{+} \in \operatorname{Irr}\left(U_{n-2} L_{n-2}^{+}\right)$to the group $R(A \times$ $U_{n-2} L_{n-2}^{+}$) also by $\vartheta_{0} \cdot \hat{\lambda}_{n-2}^{+}$. Hence,

$$
{ }^{s} \vartheta_{0} \cdot{ }^{s} \hat{\lambda}_{n-2}^{+} \in \operatorname{Irr}\left({ }^{s} R\left(A_{n-2} \times{ }^{s}\left(U_{n-2} L_{n-2}^{+}\right)\right)\right) .
$$

An elementary matrix calculation shows that $R P_{n-3}^{+} \leqslant s R\left(A_{n-2} \times{ }^{s}\left(U_{n-2} L_{n-2}^{+}\right)\right)$.

The proof now proceeds in several steps.

Step 1. We have $\left({ }^{s} \vartheta_{0} \cdot{ }^{s} \hat{\lambda}_{n-2}^{+}\right) \downarrow_{R P_{n-3}^{+}}^{s} R\left(A_{n-2} \times{ }^{s}\left(U_{n-2} L_{n-2}^{+}\right)\right)=\left(\tilde{\vartheta}_{0} \cdot \hat{\lambda}^{+}\right) \downarrow_{R P_{n-3}^{+}}^{U P_{n-3}^{+}}$.

Proof of Step 1. Let $\mathbf{u}_{n}\left(\left[v_{m-1}, \ldots, v_{0}, \ldots, v_{m-2}^{\prime}, 0\right]^{\mathrm{tr}}\right) \in R$. Then

$$
\begin{aligned}
& \left({ }^{s} \vartheta_{0} \cdot{ }^{s} \hat{\lambda}_{n-2}^{+}\right)\left(\mathbf{u}_{n}\left(\left[v_{m-1}, \ldots, v_{0}, \ldots, v_{m-2}^{\prime}, 0\right]^{\operatorname{tr}}\right)\right) \\
& \quad=\left(\vartheta_{0} \cdot \hat{\lambda}_{n-2}^{+}\right)\left(\mathbf{u}_{n}\left(\left[v_{m-1}, \ldots, v_{0}, \ldots, v_{m-2}^{\prime}, 0\right]^{\operatorname{tr}}\right)^{s}\right) \\
& \quad=\vartheta_{0}(1) \xi\left(v_{0}\right)=\vartheta_{0}(1) \cdot \hat{\lambda}^{+}\left(\mathbf{u}_{n}\left(\left[v_{m-1}, \ldots, v_{0}, \ldots, v_{m-2}^{\prime}, 0\right]^{\operatorname{tr}}\right)\right) .
\end{aligned}
$$

It follows that $\left({ }^{s} \vartheta_{0} \cdot{ }^{s} \hat{\lambda}_{n-2}^{+}\right) \downarrow_{R P_{n-3}^{+}}^{s} R\left(A_{n-2} \times^{s}\left(U_{n-2} L_{n-2}^{+}\right)\right)=\varphi \cdot\left(\hat{\lambda}^{+} \downarrow_{R P_{n-3}^{+}}^{U P_{n-3}^{+}}\right)$, where $\varphi$ is the inflation of some character of $P_{n-3}^{+}$to $R P_{n-3}^{+}$. Note that $\varphi$ is uniquely determined by its restriction to the subgroup $P_{n-3}^{+}$. We have

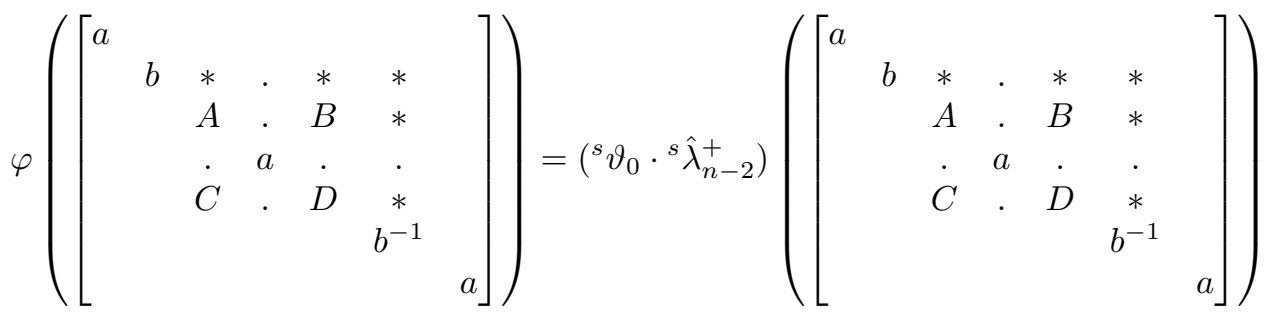

$$
\begin{aligned}
& =\left(\vartheta_{0} \cdot \hat{\lambda}_{n-2}^{+}\right)\left(\left[\begin{array}{ccccccc}
a & & & & & & \\
& b & * & \cdot & * & * & \\
& & A & \cdot & B & * & \\
& & \cdot & a & \cdot & \cdot & \\
& & C & \cdot & D & * & \\
& & & & & b^{-1} & \\
& & & & & &
\end{array}\right]^{s}\right)
\end{aligned}
$$




$$
\begin{aligned}
& =\left(\vartheta_{0} \cdot \hat{\lambda}_{n-2}^{+}\right)\left(\left[\begin{array}{ccccccc}
b & \cdot & * & \cdot & * & \cdot & * \\
& a & \cdot & \cdot & \cdot & \cdot & \cdot \\
& & A & \cdot & B & \cdot & * \\
& & \cdot & a & \cdot & \cdot & \cdot \\
& & C & \cdot & D & \cdot & * \\
& & & & & a & \cdot \\
& & & & & & b^{-1}
\end{array}\right]\right) \\
& =\vartheta_{0}\left(\left[\begin{array}{ccccc}
a & & & & \\
& A & \cdot & B & \\
& \cdot & a & \cdot & \\
& C & \cdot & D & \\
& & & & a
\end{array}\right]\right)=\tilde{\vartheta}_{0}\left(\left[\begin{array}{ccccccc}
a & & & & & & \\
& b & * & \cdot & * & * \\
& & A & \cdot & B & * \\
& \cdot & a & \cdot & \cdot \\
& C & \cdot & D & * \\
& & & & & b^{-1} & \\
& & & & & &
\end{array}\right]\right) ;
\end{aligned}
$$

thus, $\varphi \downarrow_{P_{n-3}^{+}}^{R P_{n-3}^{+}}=\tilde{\vartheta}_{0} \downarrow_{P_{n-3}^{+}}^{R P_{n-3}^{+}}$and then

$$
\left({ }^{s} \vartheta_{0} \cdot{ }^{s} \hat{\lambda}_{n-2}^{+}\right) \downarrow_{R P_{n-3}^{+}}^{s} R\left(A_{n-2} \times^{s}\left(U_{n-2} L_{n-2}^{+}\right)\right)=\tilde{\vartheta}_{0} \cdot\left(\hat{\lambda}^{+} \downarrow_{R P_{n-3}^{+}}^{U P_{-3}^{+}}\right)=\left(\tilde{\vartheta}_{0} \cdot \hat{\lambda}^{+}\right) \downarrow_{R P_{n-3}^{+}}^{U P_{n-3}^{+}} .
$$

Step 2. The character $\vartheta \cdot \hat{\lambda}^{+}$is a subcharacter of ${ }^{s} \nu \uparrow_{R Q_{K}}^{P} \downarrow_{U L_{n}^{+}}^{P}$.

Proof of Step 2. It follows from Step 1 that $\tilde{\vartheta}_{0} \cdot \hat{\lambda}^{+}$is an irreducible constituent of $\left({ }^{s} \vartheta_{0}\right.$. $\left.{ }^{s} \hat{\lambda}_{n-2}^{+}\right) \downarrow_{R P_{n-3}^{+}}^{{ }^{s} R\left(A_{n-2} \times{ }^{s}\left(U_{n-2} L_{n-2}^{+}\right)\right)_{\uparrow}}{ }_{R P_{n-3}^{+}}^{U P_{n-3}^{+}}$. Thus,

$$
\left(\tilde{\vartheta}_{0} \cdot \hat{\lambda}^{+}\right) \uparrow_{U P_{n-3}^{+}}^{U L^{+}}=\tilde{\vartheta}_{0} \uparrow_{U P_{n-3}^{+}}^{U L^{+}} \cdot \hat{\lambda}^{+}=\vartheta \cdot \hat{\lambda}^{+}
$$

is a subcharacter of $\left({ }^{s} \vartheta_{0} \cdot{ }^{s} \hat{\lambda}_{n-2}^{+}\right) \downarrow_{R P_{n-3}^{+}}^{s} R\left(A_{n-2} \times{ }^{s}\left(U_{n-2} L_{n-2}^{+}\right)\right) \uparrow_{R P_{n-3}^{+}}^{U L_{n}^{+}}$. By construction, the character $\nu \in \operatorname{Irr}\left(R Q_{K}\right)$ is given by $\nu=\left(\vartheta_{0} \cdot \hat{\lambda}_{n-2}^{+-3}\right) \uparrow_{R\left(A \times U_{n-2} L_{n-2}^{+}\right)}^{R Q_{K}}$ and hence

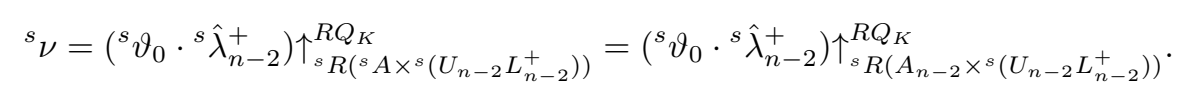

It follows from (4.12) that ${ }^{s} \vartheta_{0} \cdot s \hat{\lambda}_{n-2}^{+}$is a constituent of ${ }^{s} \nu_{\downarrow_{s} R\left(A_{n-2} \times{ }^{s}\left(U_{n-2} L_{n-2}^{+}\right)\right)}^{R Q_{K}}$. Hence, $\left({ }^{s} \vartheta_{0} \cdot s \hat{\lambda}_{n-2}^{+}\right) \downarrow_{R P_{n-3}^{+} R\left(A_{n-2} \times{ }^{s}\left(U_{n-2} L_{n-2}^{+}\right)\right)} \uparrow_{R P_{n-3}^{+}}^{U L_{n}^{+}}$is a subcharacter of ${ }^{s} \nu \downarrow^{s} R P_{n-3}^{+} \uparrow_{R P_{n-3}^{+}}^{U L_{n}^{+}}$and from the beginning of the proof of Step 2 we see that $\vartheta \cdot \hat{\lambda}^{+}$is a subcharacter of ${ }^{s} \nu \downarrow_{R P_{n-3}^{+}}^{R Q_{K}} \uparrow_{R P_{n-3}^{+}}^{U L_{n}^{+}}$. An elementary matrix calculation shows that

$$
R Q_{K} \cap U L_{n}^{+}=R P_{n-3}^{+} .
$$

Thus, Mackey's theorem implies that the character ${ }^{s} \downarrow_{\downarrow_{R P_{n-3}^{+}}^{R} \uparrow^{U}{ }_{R P_{n-3}^{+}}^{U L^{+}}}$is a subcharacter of ${ }^{s} \nu \uparrow P_{R Q_{K}}^{P} \downarrow_{U L_{n}^{+}}^{P}$ and we can conclude that $\vartheta \cdot \hat{\lambda}^{+}$is a subcharacter of ${ }^{s} \nu \uparrow_{R Q_{K}}^{P} \downarrow_{U L_{n}^{+}}^{P}$.

Step 3. We have $\left({ }^{s} \nu\right) \uparrow_{R Q_{K}}={ }^{+} \psi_{\vartheta}$.

Proof of Step 3. Write $\vartheta \cdot \hat{\lambda}^{+}=\sum_{i} m_{i} \chi_{i}$ with $\chi_{i} \in \operatorname{Irr}\left(U L_{n}^{+}\right)$. By Step 2, we have

$$
{ }_{\nu} \uparrow_{R Q_{K}}^{P} \downarrow_{U L_{n}^{+}}^{P}=\sum_{i} m_{i} \chi_{i}+(\ldots)
$$




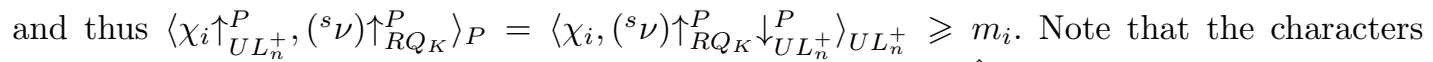
$\chi_{i} \uparrow_{U L_{n}^{+}}^{P}$ are irreducible by Clifford theory. Hence, ${ }^{+} \psi_{\vartheta}=\left(\vartheta \cdot \hat{\lambda}^{+}\right) \uparrow_{U L_{n}^{+}}^{P}$ is a subcharacter of $\left({ }^{s} \nu\right) \uparrow_{R Q_{K}}^{P}$. Computing the degrees, we get

$$
\begin{aligned}
{ }^{+} \psi_{\vartheta}(1) & =\frac{1}{2} q^{m-1}\left(q^{m-1}+1\right)(q-1) \vartheta(1) \\
& =\frac{1}{2} q^{m-1}\left(q^{m-1}+1\right)(q-1) \cdot \frac{\left|L_{n}^{+}\right|}{\left|P_{n-3}^{+}\right|} \cdot \vartheta_{0}(1) \\
& =\frac{1}{2} q^{m-1}\left(q^{m-1}+1\right)(q-1) \cdot \frac{\left(q^{m-1}-1\right) \cdot\left(q^{m-2}+1\right)}{q-1} \cdot \vartheta_{0}(1)
\end{aligned}
$$

and

$$
\begin{aligned}
\left({ }^{s} \nu\right) \uparrow_{R Q_{K}}^{P}(1) & =\frac{|P|}{\left|R Q_{K}\right|} \cdot \nu(1) \\
& =\frac{|U| \cdot\left|L_{n}\right|}{|R| \cdot(q-1) \cdot\left|P_{n-2}\right|} \cdot \frac{1}{2} q^{m-2}\left(q^{m-2}+1\right)(q-1) \cdot \vartheta_{0}(1) \\
& =\frac{1}{2} q^{m-1}\left(q^{m-1}+1\right)(q-1) \cdot \frac{\left(q^{m-1}-1\right) \cdot\left(q^{m-2}+1\right)}{q-1} \cdot \vartheta_{0}(1) .
\end{aligned}
$$

Hence, ${ }^{+} \psi_{\vartheta}(1)=\left({ }^{s} \nu\right) \uparrow_{R Q_{K}}^{P}(1)$ and then $\left({ }^{s} \nu\right) \uparrow_{R Q_{K}}^{P}={ }^{+} \psi_{\vartheta}$.

(e) Since $P_{3}$ does not have any characters of Type -, we can assume $m \geqslant 3$. We denote the inflation of $\vartheta_{0} \cdot \hat{\lambda}_{n-2}^{-} \in \operatorname{Irr}\left(U_{n-2} L_{n-2}^{-}\right)$to the group $R\left(A \times U_{n-2} L_{n-2}^{-}\right)$also by $\vartheta_{0} \cdot \hat{\lambda}_{n-2}^{-}$. Hence,

$$
{ }^{s} \vartheta_{0} \cdot{ }^{s} \hat{\lambda}_{n-2}^{-} \in \operatorname{Irr}\left({ }^{s} R\left(A_{n-2} \times{ }^{s}\left(U_{n-2} L_{n-2}^{-}\right)\right)\right) .
$$

An elementary matrix calculation shows that $R P_{n-3}^{-} \leqslant{ }^{s} R\left(A_{n-2} \times{ }^{s}\left(U_{n-2} L_{n-2}^{-}\right)\right)$.

The proof now proceeds in several steps.

Step 1. We have $\left({ }^{s} \vartheta_{0} \cdot{ }^{s} \hat{\lambda}_{n-2}^{-}\right) \downarrow_{R P_{n-3}^{-}}^{s} R\left(A_{n-2} \times^{s}\left(U_{n-2} L_{n-2}^{-}\right)\right)=\left(\tilde{\vartheta}_{0} \cdot \hat{\lambda}^{-}\right) \downarrow_{R P_{n-3}^{-}}^{U P^{-}}$.

Proof of Step 1. Let $\mathbf{v}=\left[v_{m-1}, \ldots, v_{0}, \ldots, v_{m-2}^{\prime}, 0\right]^{\text {tr }} \in \mathbb{F}_{q}^{n-2}$. Then

$$
\begin{aligned}
& \left({ }^{s} \vartheta_{0} \cdot{ }^{s} \hat{\lambda}_{n-2}^{-}\right)\left(\mathbf{u}_{n}(\mathbf{v})\right)=\left(\vartheta_{0} \cdot \hat{\lambda}_{n-2}^{-}\right)\left(\mathbf{u}_{n}(\mathbf{v})^{s}\right) \\
& \quad=\vartheta_{0}(1) \xi\left(\left(\mathbf{b}_{n-4}\left[v_{m-2}, \ldots, v_{0}, \ldots, v_{m-2}^{\prime}\right]^{\operatorname{tr}}\right)_{0}\right) \\
& \quad=\vartheta_{0}(1) \xi\left(\left(\mathbf{b}_{n-2} \mathbf{v}\right)_{0}\right)=\vartheta_{0}(1) \cdot \hat{\lambda}^{-}\left(\mathbf{u}_{n}(\mathbf{v})\right) .
\end{aligned}
$$

It follows that $\left({ }^{s} \vartheta_{0} \cdot{ }^{s} \hat{\lambda}_{n-2}^{-}\right) \downarrow_{R P_{n-3}^{-}}^{s} R\left(A_{n-2} \times^{s}\left(U_{n-2} L_{n-2}^{-}\right)\right)=\varphi \cdot\left(\hat{\lambda}^{-} \downarrow_{R P_{n-3}^{-}}^{U P_{n-3}^{-}}\right)$, where $\varphi$ is the inflation of some character of $P_{n-3}^{-}$to $R P_{n-3}^{-}$. Note that $\varphi$ is uniquely determined by its restriction to the subgroup $P_{n-3}^{-}$. We have

$$
\begin{aligned}
& \varphi\left(\mathbf{s}_{n}\left(\mathbf{b}_{n-2}^{-1}\left[\begin{array}{ccccc}
b & * & \cdot & * & * \\
& A & \cdot & B & * \\
& \cdot & a & \cdot & \cdot \\
& C & \cdot & D & * \\
& & & & b^{-1}
\end{array}\right] \mathbf{b}_{n-2}, a\right)\right) \\
& =\left({ }^{s} \vartheta_{0} \cdot{ }^{s} \hat{\lambda}_{n-2}^{-}\right)\left(\mathbf{s}_{n}\left(\mathbf{b}_{n-2}^{-1}\left[\begin{array}{ccccc}
b & * & \cdot & * & * \\
& A & \cdot & B & * \\
& \cdot & a & \cdot & \cdot \\
& C & \cdot & D & * \\
& & & & b^{-1}
\end{array}\right] \mathbf{b}_{n-2}, a\right)\right)
\end{aligned}
$$




$$
\begin{aligned}
& =\left(\vartheta_{0} \cdot \hat{\lambda}_{n-2}^{-}\right)\left(\mathbf{s}_{n}\left(\mathbf{b}_{n-2}^{-1}\left[\begin{array}{ccccc}
b & * & \cdot & * & * \\
& A & \cdot & B & * \\
& \cdot & a & \cdot & \cdot \\
& C & \cdot & D & * \\
& & & & b^{-1}
\end{array}\right] \mathbf{b}_{n-2}, a\right)\right)
\end{aligned}
$$

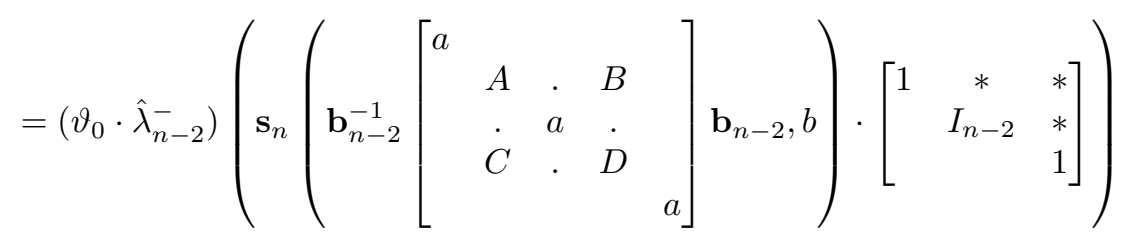

$$
\begin{aligned}
& =\vartheta_{0}\left(\mathbf{s}_{n-2}\left(\mathbf{b}_{n-4}^{-1}\left[\begin{array}{ccc}
A & \cdot & B \\
\dot{C} & a & \cdot \\
\cdot & \cdot
\end{array}\right] \mathbf{b}_{n-4}, a\right)\right) \\
& =\tilde{\vartheta}_{0}\left(\mathbf{s}_{n}\left(\mathbf{b}_{n-2}^{-1}\left[\begin{array}{ccccc}
b & * & \cdot & * & * \\
& A & \cdot & B & * \\
& \cdot & a & \cdot & \cdot \\
& C & \cdot & D & * \\
& & & & b^{-1}
\end{array}\right] \mathbf{b}_{n-2}, a\right)\right)
\end{aligned}
$$

thus, $\varphi \downarrow_{P_{n-3}^{-}}^{R P_{n-3}^{-}}=\tilde{\vartheta}_{0} \downarrow_{P_{n-3}^{-}}^{R P_{n-3}^{-}}$and then

$$
\left({ }^{s} \vartheta_{0} \cdot{ }^{s} \hat{\lambda}_{n-2}^{-}\right) \downarrow_{R P_{n-3}^{-}}^{s} R\left(A_{n-2} \times{ }^{s}\left(U_{n-2} L_{n-2}^{-}\right)\right)=\tilde{\vartheta}_{0} \cdot\left(\hat{\lambda}^{-} \downarrow_{R P_{n-3}^{-}}^{U P_{n-3}^{-}}\right)=\left(\tilde{\vartheta}_{0} \cdot \hat{\lambda}^{-}\right) \downarrow_{R P_{n-3}^{-}}^{U P_{n-3}^{-}} .
$$

Step 2. The character $\vartheta \cdot \hat{\lambda}^{-}$is a subcharacter of ${ }^{s} \nu \uparrow_{R Q_{K}}^{P} \downarrow_{U L_{n}^{-}}^{P}$.

Proof of Step 2. It follows from Step 1 that $\tilde{\vartheta}_{0} \cdot \hat{\lambda}^{-}$is an irreducible constituent of $\left({ }^{s} \vartheta_{0}\right.$. $\left.{ }^{s} \hat{\lambda}_{n-2}^{-}\right) \downarrow_{R P_{n-3}^{-}}^{s} R\left(A_{n-2} \times^{s}\left(U_{n-2} L_{n-2}^{-}\right){ }_{\uparrow} \uparrow_{R P_{n-3}^{-}}^{U P_{n-3}^{-}}\right.$. Thus,

$$
\left(\tilde{\vartheta}_{0} \cdot \hat{\lambda}^{-}\right) \uparrow_{U P_{n-3}^{-}}^{U L_{n}^{-}}=\tilde{\vartheta}_{0} \uparrow_{U P_{n-3}^{-}}^{U L_{n}^{-}} \cdot \hat{\lambda}^{-}=\vartheta \cdot \hat{\lambda}^{-}
$$

is a subcharacter of $\left({ }^{s} \vartheta_{0} \cdot s \hat{\lambda}_{n-2}^{-}\right) \downarrow_{R P_{n-3}^{-}}^{s} R\left(A_{n-2} \times^{s}\left(U_{n-2} L_{n-2}^{-}\right)\right) \uparrow_{R P_{n-3}^{-}}^{U L_{n}^{-}}$. By construction, the character $\nu \in \operatorname{Irr}\left(R Q_{K}\right)$ is given by $\nu=\left(\vartheta_{0} \cdot \hat{\lambda}_{n-2}^{--3}\right) \uparrow_{R\left(A \times U_{n-2} L_{n-2}^{-}\right)}^{R Q_{K}}$ and hence

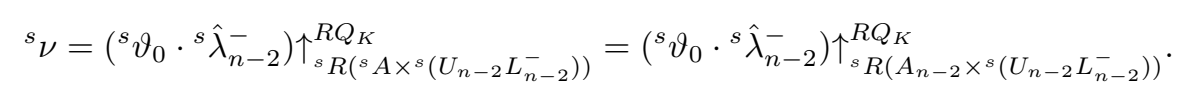

It follows from (4.13) that ${ }^{s} \vartheta_{0} \cdot{ }^{s} \hat{\lambda}_{n-2}^{-}$is a constituent of ${ }^{s} \nu \downarrow_{s}^{R Q_{K}}{ }_{R\left(A_{n-2} \times{ }^{s}\left(U_{n-2} L_{n-2}^{-}\right)\right)}$. Hence, $\left({ }^{s} \vartheta_{0} \cdot{ }^{s} \hat{\lambda}_{n-2}^{-}\right) \downarrow_{R P_{n-3}^{-} R\left(A_{n-2} \times{ }^{s}\left(U_{n-2} L_{n-2}^{-}\right)\right)} \uparrow_{R P_{n-3}^{-}}^{U L_{n}^{-}}$is a subcharacter of ${ }^{s} \nu \downarrow_{R P_{n-3}^{-}}^{R Q_{K}} \uparrow_{R P_{n-3}^{-}}^{U L_{n}^{-}}$and from the beginning of the proof of Step 2 we see that $\vartheta \cdot \hat{\lambda}^{-}$is a subcharacter of ${ }^{s} \nu \downarrow_{R P_{n-3}^{-}}^{R Q_{K}} \uparrow_{R P_{n-3}^{-}}^{U L_{n}^{-}}$. An elementary matrix calculation shows that

$$
R Q_{K} \cap U L_{n}^{-}=R P_{n-3}^{-} .
$$

Thus, Mackey's theorem implies that the character ${ }^{s} \nu_{\downarrow} \downarrow_{R P_{n-3}^{-}}^{R Q_{K}} \uparrow_{R P_{n-3}^{-}}^{U L_{n}^{-}}$is a subcharacter of ${ }^{s} \nu \uparrow P_{R Q_{K}}^{P} \downarrow_{U L_{n}^{-}}^{P}$ and we can conclude that $\vartheta \cdot \hat{\lambda}^{-}$is a subcharacter of ${ }^{s} \nu \uparrow_{R Q_{K}}^{P} \downarrow_{U L_{n}^{-}}^{P}$. 
Step 3. We have $\left({ }^{s} \nu\right) \uparrow_{R Q_{K}}^{P}={ }^{-} \psi_{\vartheta}$.

Proof of Step 3. Write $\vartheta \cdot \hat{\lambda}^{-}=\sum_{i} m_{i} \chi_{i}$ with $\chi_{i} \in \operatorname{Irr}\left(U L_{n}^{-}\right)$. By Step 2, we have

$$
{ }^{s} \uparrow_{R Q_{K}}^{P} \downarrow_{U L_{n}^{-}}^{P}=\sum_{i} m_{i} \chi_{i}+(\ldots)
$$

and thus $\left\langle\chi_{i} \uparrow_{U L_{n}^{-}}^{P},\left({ }^{s} \nu\right) \uparrow_{R Q_{K}}^{P}\right\rangle_{P}=\left\langle\chi_{i},\left({ }^{s} \nu\right) \uparrow_{R Q_{K}{ }^{P}}^{P}{ }_{U L_{n}^{-}}\right\rangle_{U L_{n}^{-}} \geqslant m_{i}$. Note that the characters $\chi_{i} \uparrow_{U L_{n}^{-}}^{P}$ are irreducible by Clifford theory. Hence, ${ }^{-} \psi_{\vartheta}=\left(\vartheta \cdot \hat{\lambda}^{-}\right) \uparrow_{U L_{n}^{-}}^{P}$ is a subcharacter of $\left({ }^{s} \nu\right) \uparrow_{R Q_{K}}^{P}$. Computing the degrees, we get

$$
\begin{aligned}
{ }^{-} \psi_{\vartheta}(1) & =\frac{1}{2} q^{m-1}\left(q^{m-1}-1\right)(q-1) \vartheta(1) \\
& =\frac{1}{2} q^{m-1}\left(q^{m-1}-1\right)(q-1) \cdot \frac{\left|L_{n}^{-}\right|}{\left|P_{n-3}^{-}\right|} \cdot \vartheta_{0}(1) \\
& =\frac{1}{2} q^{m-1}\left(q^{m-1}-1\right)(q-1) \cdot \frac{\left(q^{m-1}+1\right) \cdot\left(q^{m-2}-1\right)}{q-1} \cdot \vartheta_{0}(1)
\end{aligned}
$$

and

$$
\begin{aligned}
\left({ }^{s} \nu\right) \uparrow_{R Q_{K}}^{P}(1) & =\frac{|P|}{\left|R Q_{K}\right|} \cdot \nu(1) \\
& =\frac{|U| \cdot\left|L_{n}\right|}{|R| \cdot(q-1) \cdot\left|P_{n-2}\right|} \cdot \frac{1}{2} q^{m-2}\left(q^{m-2}-1\right)(q-1) \cdot \vartheta_{0}(1) \\
& =\frac{1}{2} q^{m-1}\left(q^{m-1}-1\right)(q-1) \cdot \frac{\left(q^{m-1}+1\right) \cdot\left(q^{m-2}-1\right)}{q-1} \cdot \vartheta_{0}(1) .
\end{aligned}
$$

Hence, ${ }^{-} \psi_{\vartheta}(1)=\left({ }^{s} \nu\right) \uparrow_{R Q_{K}}^{P}(1)$ and then $\left({ }^{s} \nu\right) \uparrow_{R Q_{K}}^{P}={ }^{-} \psi_{\vartheta}$.

\section{Restriction of the Steinberg character}

We use the setting and notation from the previous sections. In particular, we fix an odd integer $n=2 m+1$ and let $G=G_{n}=\mathrm{SO}_{2 m+1}(q)$. The restriction of the Steinberg character $\mathrm{St}_{G}$ to the parabolic subgroup $P=P_{n}$ was already investigated by Schmölzer in [13]. See also $[\mathbf{1}, \S 3]$ for a comparison to the symplectic case.

Proposition 5.1 [13, Corollary 2.3.8]. Let $n \geqslant 5$ and $L^{\prime}:=L_{n}^{\prime} \cong \mathrm{SO}_{n-2}(q)$. For each $\sigma \in \operatorname{Irr}\left(L^{\prime}\right)$ we denote its trivial extension to $L$ also by $\sigma$. Then

$$
\begin{aligned}
\sigma \uparrow_{L}^{P}= & { }^{1} \psi_{\sigma}+\sum_{\mu \in \operatorname{Irr}\left(P_{n-2}\right)}\left\langle\sigma \downarrow_{P_{n-2}}^{L^{\prime}}, \mu\right\rangle_{P_{n-2}}{ }^{0} \psi_{\mu} \\
& +\sum_{\vartheta \in \operatorname{Irr}\left(L_{n}^{+}\right)}\left\langle\sigma \downarrow_{L_{n}^{+}}^{L}, \vartheta\right\rangle_{L_{n}^{+}}{ }^{+} \psi_{\vartheta}+\sum_{\vartheta \in \operatorname{Irr}\left(L_{n}^{-}\right)}\left\langle\sigma \downarrow_{L_{n}^{-}}^{L}, \vartheta\right\rangle_{L_{n}^{-}}{ }^{-} \psi_{\vartheta} .
\end{aligned}
$$

Proof. This is analogous to [1, Proposition 3.2].

We are interested in the decomposition of $\mathrm{St}_{G} \downarrow{ }_{P}^{G}$ into irreducible characters. The following corollary reduces this problem to calculations with characters of subgroups of $L$. In $\S \S 7$ and 8 , this reduction will be used to get a complete description of the restriction of the Steinberg character for $n=5$ and to get a partial description for $n=7$. 
Corollary $5.2[\mathbf{1 3}$, Corollary 2.3.9]. Suppose $n \geqslant 5$. Then

$$
\begin{aligned}
\mathrm{St}_{G \downarrow} \downarrow_{P}^{G}= & { }^{1} \psi_{\mathrm{St}_{L}}+\sum_{\mu \in \operatorname{Irr}\left(P_{n-2}\right)}\left\langle\mathrm{St}_{\left.L^{\prime} \downarrow_{P_{n-2}}^{L^{\prime}}, \mu\right\rangle_{P_{n-2}}{ }^{0} \psi_{\mu}}\left\langle\operatorname{St}_{L \downarrow} \downarrow_{L_{n}^{+}}^{L}, \vartheta\right\rangle_{L_{n}^{+}}{ }^{+} \psi_{\vartheta}+\sum_{\vartheta \in \operatorname{Irr}\left(L_{n}^{-}\right)}\left\langle\mathrm{St}_{L} \downarrow_{L_{n}^{-}}^{L}, \vartheta\right\rangle_{L_{n}^{-}}{ }^{-} \psi_{\vartheta} .\right.
\end{aligned}
$$

Proof. This follows from [3, Proposition 6.3.3] and Proposition 5.1.

\section{Character values on $U$}

As before, we fix an odd integer $n=2 m+1$ and let $G=G_{n}=\mathrm{SO}_{n}(q)$. We determine the values of the irreducible characters of the parabolic subgroup $P=P_{n}$ on those conjugacy classes of $P$ which are contained in $U=U_{n}$. In this section we always assume $n \geqslant 5$.

Let $\left\{e_{m-1}, \ldots, e_{1}, e_{0}, e_{1}^{\prime}, \ldots, e_{m-1}^{\prime}\right\}$ be the standard basis of $\mathbb{F}_{q}^{n-2}$ as in $\S 2.1$. The conjugation action of $L$ on the normal abelian subgroup $U$ is given by $\mathbf{s}_{n}(\mathbf{x}, a) \mathbf{u}_{n}(\mathbf{v})=\mathbf{u}_{n}(a \mathbf{x v})$. Since $\mathbf{x} \in \mathrm{SO}_{n-2}(q)$, the values $Q_{n-2}(\mathbf{v})$ and $Q_{n-2}(a \mathbf{x v})$ differ only by a non-zero factor which is a square in $\mathbb{F}_{q}^{\times}$.

We define $\mathbf{v}_{0}, \mathbf{v}_{1}, \mathbf{v}_{2} \in \mathbb{F}_{q}^{n-2}$ by $\mathbf{v}_{0}:=e_{m-1}, \mathbf{v}_{1}:=e_{0}, \mathbf{v}_{2}:=e_{m-1}+\nu^{\prime \prime} e_{m-1}^{\prime}$, where $\nu^{\prime \prime} \in \mathbb{F}_{q}^{\times}$ is a non-square if $q$ is odd and $\nu^{\prime \prime}=1$ if $q$ is even. We write $\mathbf{z}_{j}:=\mathbf{u}_{n}\left(\mathbf{v}_{j}\right)(j=0,1,2)$ for the corresponding elements of $U$. We know from Brauer's permutation lemma [10, Corollary 6.33] and the results in $\S 3.2$ that the number of conjugacy classes of $P$ which are contained in $U$ is four.

The set $\left\{1, \mathbf{z}_{0}, \mathbf{z}_{1}, \mathbf{z}_{2}\right\}$ is a full set of representatives for the conjugacy classes of $P$ which are contained in $U$. For odd $q$ this already follows from $Q_{n-2}\left(\mathbf{v}_{0}\right)=0, Q_{n-2}\left(\mathbf{v}_{1}\right)=1$ and $Q_{n-2}\left(\mathbf{v}_{2}\right)=\nu^{\prime \prime}$. For even $q$ the definition of $\mathrm{SO}_{n-2}(q)$ and $Q_{n-2}$ implies that $e_{0}$ is a common eigenvector of all $\mathbf{x} \in \mathrm{SO}_{n-2}(q)$, so that $\mathbf{z}_{1}$ is not conjugate to $\mathbf{z}_{2}$ in $P$. Now, also in this case, the values $Q_{n-2}\left(\mathbf{v}_{0}\right)=0, Q_{n-2}\left(\mathbf{v}_{1}\right)=1$ and $Q_{n-2}\left(\mathbf{v}_{2}\right)=\nu^{\prime \prime}$ imply that $\left\{1, \mathbf{z}_{0}, \mathbf{z}_{1}, \mathbf{z}_{2}\right\}$ is a full set of representatives for the conjugacy classes of $P$ which are contained in $U$. By a straightforward calculation, similar to those in $\S 3.2$, one can compute the centralizers $C_{P}\left(\mathbf{z}_{0}\right)$, $C_{P}\left(\mathbf{z}_{1}\right)$ and their orders explicitly. From these orders one gets the size of the $P$-conjugacy class containing $\mathbf{z}_{2}$ and then $\left|C_{P}\left(\mathbf{z}_{2}\right)\right|$. The orders of the centralizers are

$$
\begin{aligned}
& \left|C_{P}\left(\mathbf{z}_{0}\right)\right|=q^{m^{2}}(q-1)\left(q^{2 m-4}-1\right)\left(q^{2 m-6}-1\right) \ldots\left(q^{2}-1\right), \\
& \left|C_{P}\left(\mathbf{z}_{1}\right)\right|= \begin{cases}2 q^{m^{2}-m+1}\left(q^{m-1}-1\right)\left(q^{2 m-4}-1\right) \ldots\left(q^{2}-1\right) & \text { for odd } q, \\
q^{m^{2}}\left(q^{2 m-2}-1\right)\left(q^{2 m-4}-1\right) \ldots\left(q^{2}-1\right) & \text { for even } q,\end{cases} \\
& \left|C_{P}\left(\mathbf{z}_{2}\right)\right|= \begin{cases}2 q^{m^{2}-m+1}\left(q^{m-1}+1\right)\left(q^{2 m-4}-1\right) \ldots\left(q^{2}-1\right) & \text { for odd } q, \\
q^{m^{2}}\left(q^{2 m-4}-1\right)\left(q^{2 m-6}-1\right) \ldots\left(q^{2}-1\right) & \text { for even } q .\end{cases}
\end{aligned}
$$

For $j=0,1,2$, let $c_{j}$ be the $P$-conjugacy class containing $\mathbf{z}_{j}$ and $C_{j}$ the $G$-conjugacy class containing $\mathbf{z}_{j}$. To express the dependency on $n$, we also write $c_{j}^{(n)}$ and $C_{j}^{(n)}$, respectively.

Remark 2. For $n \geqslant 5$ the $G$-conjugacy classes $C_{0}, C_{1}, C_{2}$ are pairwise distinct.

Proof. It follows from Lemma 4.1 (a) that a full set of $P$-P-double coset representatives in $G$ is given by $\{1, s, t\}$, where $s$ and $t$ are the elements defined in (2.4). Furthermore, Lemma 4.1(d)(iii) implies that ${ }^{s} c_{j} \cap U=\emptyset$ for $j=1,2$ and ${ }^{s} c_{0} \cap U \subseteq c_{0}$ and ${ }^{t} c_{j} \cap U=\emptyset$ for all $j$, so the claim follows. 
Lemma 6.1. Let $n \geqslant 5$. Let $\mu$ be a character of $P_{n-2}$ and $\vartheta$ a character of $L_{n}^{+}$or $L_{n}^{-}$. The values the irreducible characters of $P$ take on the unipotent elements $\mathbf{z}_{0}, \mathbf{z}_{1}$ and $\mathbf{z}_{2}$ are given in Tables 1 and 2.

Proof. We use the notation from $\S \S 3.2$ and 3.3, except for the fact that in this proof we denote irreducible characters of $P_{n-2}$ also by $\vartheta$ (and not by $\mu$ ) to treat all characters in a uniform way. Let $\varepsilon \in\{0,+,-\}$ and $j \in\{0,1,2\}$ and let $\vartheta \in \operatorname{Irr}\left(P_{n-2}\right)$ if $\varepsilon=0$ and $\vartheta \in \operatorname{Irr}\left(L_{n}^{ \pm}\right)$ if $\varepsilon= \pm$. Since $U \unlhd P$, we get from the definition of induced characters and the construction of ${ }^{\varepsilon} \psi_{\vartheta}$ that

$$
{ }^{\varepsilon} \psi_{\vartheta}\left(\mathbf{z}_{j}\right)=\frac{1}{\left|I^{\varepsilon}\right|} \sum_{x \in P} \vartheta(1) \lambda^{\varepsilon}\left(x \mathbf{z}_{j} x^{-1}\right)=\frac{\left|C_{P}\left(\mathbf{z}_{j}\right)\right| \cdot \vartheta(1)}{\left|I^{\varepsilon}\right|} \sum_{\mathbf{z} \in c_{j}} \lambda^{\varepsilon}(\mathbf{z}) .
$$

Since $\left|C_{P}\left(\mathbf{z}_{j}\right)\right|$ and $\left|I^{\varepsilon}\right|$ are known, we only need to determine the sum of character values on the right-hand side of (6.2).

The subgroup $A \leqslant L$ defined in $\S 2.3$ acts regularly on the $A$-suborbits of each of the

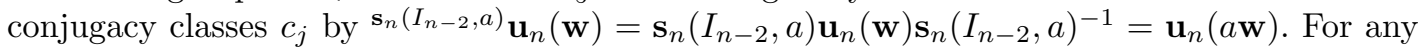
vector $\mathbf{w}=\left[w_{m-1}, \ldots, w_{1}, w_{0}, w_{1}^{\prime}, \ldots, w_{m-1}^{\prime}\right]^{\operatorname{tr}} \in \mathbb{F}_{q}^{n-2}$, we have

$$
\sum_{a \in \mathbb{F}_{q}^{\times}} \lambda^{0}\left(\mathbf{u}_{n}(a \mathbf{w})\right)=\sum_{a \in \mathbb{F}_{q}^{\times}} \xi\left(a w_{m-1}^{\prime}\right)= \begin{cases}q-1 & \text { if } w_{m-1}^{\prime}=0, \\ -1 & \text { if } w_{m-1}^{\prime} \neq 0,\end{cases}
$$

and

$$
\sum_{a \in \mathbb{F}_{q}^{\times}} \lambda^{+}\left(\mathbf{u}_{n}(a \mathbf{w})\right)=\sum_{a \in \mathbb{F}_{q}^{\times}} \xi\left(a w_{0}\right)= \begin{cases}q-1 & \text { if } w_{0}=0 \\ -1 & \text { if } w_{0} \neq 0 .\end{cases}
$$

We set ${ }^{0} N_{j}:=\left|\left\{\mathbf{u}_{n}(\mathbf{w}) \in c_{j} \mid w_{m-1}^{\prime}=0\right\}\right|$ and ${ }^{+} N_{j}:=\left|\left\{\mathbf{u}_{n}(\mathbf{w}) \in c_{j} \mid w_{0}=0\right\}\right|$. By partitioning $c_{j}$ into regular $A$-suborbits, we have

$$
\sum_{\mathbf{z} \in c_{j}} \lambda^{\varepsilon}(\mathbf{z})=\frac{q-1}{q-1} \cdot{ }^{\varepsilon} N_{j}+\frac{\left|c_{j}\right|-{ }^{\varepsilon} N_{j}}{q-1} \cdot(-1)=\frac{1}{q-1}\left(q \cdot{ }^{\varepsilon} N_{j}-\left|c_{j}\right|\right) .
$$

TABLE 1. Character values on $U$ for odd $q$.

\begin{tabular}{llll}
\hline & \multicolumn{1}{c}{$\mathbf{z}_{0}$} & \multicolumn{1}{c}{$\mathbf{z}_{1}$} & \multicolumn{1}{c}{$\mathbf{z}_{2}$} \\
\hline${ }^{0} \psi_{\mu}$ & $-\mu(1)$ & $\left(q^{m-1}-1\right) \mu(1)$ & $-\left(q^{m-1}+1\right) \mu(1)$ \\
${ }^{+} \psi_{\vartheta}$ & $\frac{1}{2} q^{m-1}(q-1) \vartheta(1)$ & $-q^{m-1} \vartheta(1)$ & 0 \\
$-{ }_{\vartheta}$ & $-\frac{1}{2} q^{m-1}(q-1) \vartheta(1)$ & 0 & $q^{m-1} \vartheta(1)$ \\
\hline
\end{tabular}

TABLE 2. Character values on $U$ for even $q$.

\begin{tabular}{llll}
\hline & \multicolumn{1}{c}{$\mathbf{z}_{0}$} & \multicolumn{1}{c}{$\mathbf{z}_{1}$} & $\mathbf{z}_{2}$ \\
\hline${ }^{0} \psi_{\mu}$ & $-\mu(1)$ & $\left(q^{2 m-2}-1\right) \mu(1)$ & $-\mu(1)$ \\
${ }^{+} \psi_{\vartheta}$ & $\frac{1}{2} q^{m-1}(q-1) \vartheta(1)$ & $-\frac{1}{2} q^{m-1}\left(q^{m-1}+1\right) \vartheta(1)$ & $-\frac{1}{2} q^{m-1} \vartheta(1)$ \\
$-\psi_{\vartheta}$ & $-\frac{1}{2} q^{m-1}(q-1) \vartheta(1)$ & $-\frac{1}{2} q^{m-1}\left(q^{m-1}-1\right) \vartheta(1)$ & $\frac{1}{2} q^{m-1} \vartheta(1)$ \\
\hline
\end{tabular}


Suppose $\varepsilon=0$. For $j=0$, we have

$$
\begin{aligned}
{ }^{0} N_{0} & =\mid\left\{\mathbf{w} \in \mathbb{F}_{q}^{n-2} \backslash\{0\} \mid Q_{n-2}(\mathbf{w})=0 \text { and } w_{m-1}^{\prime}=0\right\} \mid \\
& =q \cdot\left|c_{0}^{(n-2)}\right|+q-1=q \cdot\left(q^{2 m-4}-1\right)+q-1=q^{2 m-3}-1,
\end{aligned}
$$

so that (6.3) evaluates to -1 . Next, let $j=1$. If $q$ is odd, we have

$$
\begin{aligned}
{ }^{0} N_{1} & =\mid\left\{\mathbf{w} \in \mathbb{F}_{q}^{n-2} \mid Q_{n-2}(\mathbf{w}) \text { is a square in } \mathbb{F}_{q}^{\times} \text {and } w_{m-1}^{\prime}=0\right\} \mid \\
& =q \cdot\left|c_{1}^{(n-2)}\right|=\frac{q-1}{2} q^{m-1}\left(q^{m-2}+1\right),
\end{aligned}
$$

giving $\frac{1}{2} q^{m-1}(q-1)$ as the right-hand side of (6.3). If $q$ is even, then ${ }^{0} N_{1}=q-1$, and the right-hand side of (6.3) evaluates to $q-1$. This gives the values of ${ }^{0} \psi_{\vartheta}$ on $\mathbf{z}_{0}$ and $\mathbf{z}_{1}$. The values of ${ }^{0} \psi_{\vartheta}$ on $\mathbf{z}_{2}$ follow from row orthogonality relations for the characters of $U$.

Suppose $\varepsilon=+$. By [11, Lemma 6.10], for odd $q$ and even $q$, we have ${ }^{+} N_{0}=q^{m-2}\left(q^{m-1}+\right.$ $q-1)-1$, giving $q^{m-1}-1$ for (6.3). Similarly, we obtain ${ }^{+} N_{1}=((q-1) / 2) q^{m-2}\left(q^{m-1}-1\right)$ for odd $q$ and hence the right-hand side of (6.3) evaluates to $-q^{m-1}$ in this case. However, for even $q$ we obtain ${ }^{+} N_{1}=0$, as the only vector $\mathbf{w}$ which is a scalar multiple of $e_{0}$ with $w_{0}=0$ is the zero vector. Therefore, (6.3) evaluates to -1 . The values of ${ }^{+} \psi_{\vartheta}$ on $\mathbf{z}_{2}$ can be determined again with the help of row orthogonality relations.

Suppose $\varepsilon=-$. By the column orthogonality relations for $\operatorname{Irr}(U)$, the sum of all coefficients of $\mu(1)$ and $\vartheta(1)$ in each column of Tables 1 and 2 has to be -1 . This gives the values of ${ }^{-} \psi_{\vartheta}$ and completes the proof.

Remark 3. Let $n \geqslant 5$. Each character $\chi$ of $P=P_{n}$ can be written uniquely as $\chi=$ ${ }^{1} \chi+{ }^{0} \chi+{ }^{+} \chi+{ }^{-} \chi$, where ${ }^{\varepsilon} \chi$ is the sum of the constituents of $\chi$ of Type $\varepsilon$ and $\varepsilon \in\{0,1,+,-\}$. So, each ${ }^{\varepsilon} \chi$ is of the form ${ }^{\varepsilon} \psi_{\vartheta^{\varepsilon}}$ for some not necessarily irreducible character $\vartheta^{\varepsilon}$. We call ${ }^{\varepsilon} \chi$ the Type $\varepsilon$ component of $\chi$. Lemma 6.1 allows us to reconstruct the degrees ${ }^{\varepsilon} \chi(1)$ or equivalently the degrees $\vartheta^{\varepsilon}(1)$ from the values of $\chi$ on the conjugacy classes of $P$ which are contained in $U$ as follows: for odd $q$ we set

$$
M:=\left[\begin{array}{cccc}
1 & q^{2 m-2}-1 & \frac{q^{m-1}}{2}\left(q^{m-1}+1\right)(q-1) & \frac{q^{m-1}}{2}\left(q^{m-1}-1\right)(q-1) \\
1 & -1 & \frac{q^{m-1}}{2}(q-1) & -\frac{q^{m-1}}{2}(q-1) \\
1 & q^{m-1}-1 & -q^{m-1} & 0 \\
1 & -\left(q^{m-1}+1\right) & 0 & q^{m-1}
\end{array}\right]
$$

and for even $q$ we define

$$
M:=\left[\begin{array}{cccc}
1 & q^{2 m-2}-1 & \frac{q^{m-1}}{2}\left(q^{m-1}+1\right)(q-1) & \frac{q^{m-1}}{2}\left(q^{m-1}-1\right)(q-1) \\
1 & -1 & \frac{q^{m-1}}{2}(q-1) & -\frac{q^{m-1}}{2}(q-1) \\
1 & q^{2 m-2}-1 & -\frac{q^{m-1}}{2}\left(q^{m-1}+1\right) & -\frac{q^{m-1}}{2}\left(q^{m-1}-1\right) \\
1 & -1 & -\frac{q^{m-1}}{2} & \frac{q^{m-1}}{2}
\end{array}\right] .
$$

Because $\operatorname{det}(M)=q^{4 m-2} \neq 0$ for odd $q$ and $\operatorname{det}(M)=\frac{1}{2} q^{5 m-3} \neq 0$ for even $q$, we get from Lemma 6.1 for all odd $q$ and all even $q$ :

$$
\left[\begin{array}{l}
\vartheta^{1}(1) \\
\vartheta^{0}(1) \\
\vartheta^{+}(1) \\
\vartheta^{-}(1)
\end{array}\right]=M^{-1}\left[\begin{array}{c}
\chi(1) \\
\chi\left(\mathbf{z}_{0}\right) \\
\chi\left(\mathbf{z}_{1}\right) \\
\chi\left(\mathbf{z}_{2}\right)
\end{array}\right]
$$




\section{Restrictions of unipotent characters of $\mathrm{SO}_{5}(q)$}

When we speak of a unipotent character we always mean an irreducible character. Recall (see $[3, \S 13.8]$ ) that for $n=2 m+1$ the unipotent characters of the group $G=\operatorname{SO}_{n}(q)$ are parameterized by symbols

$$
\Lambda=\left(\begin{array}{ccccc}
\lambda_{1} & \lambda_{2} & \lambda_{3} & \ldots & \lambda_{r} \\
\mu_{1} & \mu_{2} & \ldots & \mu_{s}
\end{array}\right)
$$

for which $0 \leqslant \lambda_{1}<\ldots<\lambda_{r}$ and $0 \leqslant \mu_{1}<\ldots<\mu_{s}$ are strictly increasing sequences of nonnegative integers and the second sequence may be empty. Furthermore, the difference $r-s$ is odd and $\sum \lambda_{i}+\sum \mu_{j}-\left\lfloor((r+s-1) / 2)^{2}\right\rfloor=m$. We call $r-s$ the defect of $\Lambda$ and $m$ its rank. There is a bijection (see $[\mathbf{3}, \S 11.4]$ ) between the set of symbols of rank $m$ and defect $d=2 d^{\prime}+1$ and the set of bipartitions $(\alpha, \beta)$ such that $|\alpha|+|\beta|=m-\left(d^{\prime 2}+d^{\prime}\right)$. Via this bijection we identify each symbol $\Lambda$ with the triple $[\alpha, \beta, d]$ consisting of its corresponding bipartition and its defect. We write $\chi_{\Lambda}$ for the unipotent character of $G$ corresponding to the symbol $\Lambda$.

In this section we determine the decomposition into irreducible characters of the restrictions of the unipotent characters of $G_{5}=\mathrm{SO}_{5}(q)$ to the maximal parabolic subgroup $P_{5}$. In the whole section we assume that $m=2$ and $n=2 m+1=5$. We set $G:=\operatorname{SO}_{5}(q), P:=P_{5}$, $L:=L_{5}$ and $L^{ \pm}:=L_{5}^{ \pm}$for brevity. The degrees of the unipotent characters of $\operatorname{SO}_{5}(q)$ and their labels are given in Table 3 .

As in $\S 6$, we denote the $P$-conjugacy class of the unipotent element $\mathbf{z}_{j}$ by $c_{j}$ for $j=0,1,2$. For odd prime powers $q$ the conjugacy classes and the values of the unipotent characters of $G=\mathrm{SO}_{5}(q)$ were computed by Frank Lübeck (private communication, 2013). The group $G$ has exactly five unipotent conjugacy classes and the orders of the centralizer in $G$ of representatives for these classes are $|G|, q^{4}\left(q^{2}-1\right), 2 q^{3}(q-1), 2 q^{3}(q+1)$ and $q^{2}$, respectively. Remark 2 and the centralizer orders (6.1) determine the fusion of the classes $c_{0}, c_{1}$ and $c_{2}$ into the unipotent classes of $G$ uniquely so that we can read off the values of the unipotent characters of $G$ on the elements $\mathbf{z}_{0}, \mathbf{z}_{1}$ and $\mathbf{z}_{2}$ in Table 4 for odd $q$ from Lübeck's data. In the table zeros are replaced by dots.

TABLE 3. Labels and degrees of the unipotent characters of $\mathrm{SO}_{5}(q)$.

\begin{tabular}{llllll}
\hline Bipartition & Symbol & Degree & Bipartition & Symbol & Degree \\
\hline$[2,-, 1]$ & $\left(\begin{array}{c}2 \\
-\end{array}\right)$ & 1 & {$[1,1,1]$} & $\left(\begin{array}{c}02 \\
1\end{array}\right)$ & $\frac{1}{2} q(q+1)^{2}$ \\
{$[-,-, 3]$} & $\left(\begin{array}{c}012 \\
-\end{array}\right)$ & $\frac{1}{2} q(q-1)^{2}$ & {$[-, 2,1]$} & $\left(\begin{array}{c}01 \\
2\end{array}\right)$ & $\frac{1}{2} q\left(q^{2}+1\right)$ \\
{$\left[1^{2},-, 1\right]$} & $\left(\begin{array}{c}12 \\
0\end{array}\right)$ & $\frac{1}{2} q\left(q^{2}+1\right)$ & {$\left[-, 1^{2}, 1\right]$} & $\left(\begin{array}{c}012 \\
12\end{array}\right)$ & $q^{4}$ \\
\hline
\end{tabular}

TABLE 4. Values $\chi_{\Lambda}\left(\mathbf{z}_{j}\right)$ of unipotent characters of $\mathrm{SO}_{5}(q)$.

\begin{tabular}{llllllll}
\hline & \multicolumn{3}{c}{$q$ odd } & & \multicolumn{3}{c}{$q$ even } \\
\cline { 2 - 3 } \cline { 6 - 8 }$\Lambda$ & $\mathbf{z}_{0}$ & $\mathbf{z}_{1}$ & $\mathbf{z}_{2}$ & & $\mathbf{z}_{0}$ & \multicolumn{1}{c}{$\mathbf{z}_{1}$} & $\mathbf{z}_{2}$ \\
\hline$[-,-, 3]$ & $-\frac{1}{2} q(q-1)$ &. & $q$ & $-\frac{1}{2} q(q-1)$ & $-\frac{1}{2} q(q-1)$ & $\frac{1}{2} q$ \\
{$\left[1^{2},-, 1\right]$} & $-\frac{1}{2} q(q-1)$ & $q$ &. & $-\frac{1}{2} q(q-1)$ & $\frac{1}{2} q(q+1)$ & $\frac{1}{2} q$ \\
{$[1,1,1]$} & $\frac{1}{2} q(q+1)$ & $q$ &. & $\frac{1}{2} q(q+1)$ & $\frac{1}{2} q(q+1)$ & $\frac{1}{2} q$ \\
{$[-, 2,1]$} & $\frac{1}{2} q(q+1)$ &. & $q$ & $\frac{1}{2} q(q+1)$ & $-\frac{1}{2} q(q-1)$ & $\frac{1}{2} q$ \\
\hline
\end{tabular}


To determine the values of the unipotent characters of $G$ on $\mathbf{z}_{j}$ for even $q$, we identify $G=\mathrm{SO}_{5}(q)$ with $\mathrm{Sp}_{4}(q)$ via the isomorphism described in Remark 1. The conjugacy classes and the irreducible characters of $\operatorname{Sp}_{4}(q)$ for even $q$ were determined by Enomoto [5]. Using the notation for the unipotent conjugacy classes and the representatives in [5], we see that $\mathbf{z}_{0} \in A_{31}, \mathbf{z}_{1} \in A_{2}$ and $\mathbf{z}_{2} \in A_{32}$. Now the character table of $\operatorname{Sp}_{4}(q)$ in [5] or CHEVIE [6] gives the values in Table 4 for even $q$.

Additionally, we have the values $\chi_{[2,-, 1]}\left(\mathbf{z}_{j}\right)=1$ for all $j$ for the trivial character and $\chi_{\left[-, 1^{2}, 1\right]}\left(\mathbf{z}_{j}\right)=0$ for all $j$ for the Steinberg character. To describe the restriction of the irreducible characters of $G=\mathrm{SO}_{5}(q)$ to the parabolic subgroup $P$, we collect some information on the conjugacy classes and irreducible characters of some subgroups of $G$.

REMARK 4. (a) The parabolic subgroup $P_{3} \cong q:(q-1)$ of $L^{\prime}:=L_{5}^{\prime} \cong \mathrm{SO}_{3}(q)$ is a Borel subgroup and has $q-1$ linear characters and a unique non-linear irreducible character, which we denote by $\mu$. Its degree is $\mu(1)=q-1$.

(b) By [14, Theorem 11.4], the group $L^{ \pm} \cong \mathrm{GO}_{2}^{ \pm}(q)$ is dihedral of order $2(q \mp 1)$. More specifically: $L^{ \pm}$has a cyclic normal subgroup $K^{ \pm}$of index 2 , and an outer involution acts on $K^{ \pm}$by inverting each element. For odd $q$ we have $K^{ \pm}=L^{ \pm} \cap L^{\prime}$. In other words: $K^{ \pm}=\mathrm{SO}_{2}^{ \pm}(q)$ when we identify $L^{ \pm}$with $\mathrm{GO}_{2}^{ \pm}(q)$ and $q$ is odd.

For odd $q$ the group $L^{ \pm}$has four linear characters $1_{L^{ \pm}}, \nu_{1}^{ \pm}, \nu_{2}^{ \pm}, \nu_{3}^{ \pm}$and $(q \mp 1) / 2-1$ irreducible characters $\chi_{j}^{ \pm}$of degree 2 and we choose the numbering so that $K^{ \pm} \leqslant \operatorname{ker}\left(\nu_{1}^{ \pm}\right)$and $K^{ \pm} \nless \operatorname{ker}\left(\nu_{2}^{ \pm}\right), \operatorname{ker}\left(\nu_{3}^{ \pm}\right)$. We will see in Theorem 7.1 that exactly one of the characters $\nu_{2}^{ \pm}$, $\nu_{3}^{ \pm}$is a constituent of $\mathrm{St}_{L} \downarrow_{L^{ \pm}}^{L}$ and we choose the notation so that $\nu_{3}^{ \pm}$is this constituent. The group $L^{ \pm}$has exactly two conjugacy classes which are not contained in $K^{ \pm}$. Both of them consist of involutions and have size $(q \mp 1) / 2$.

For even $q$ the group $L^{ \pm}$has two linear characters $1_{L^{ \pm}}, \nu_{1}^{ \pm}$and $(q-1 \mp 1) / 2$ irreducible characters $\chi_{j}^{ \pm}$of degree 2 and we have $K^{ \pm} \leqslant \operatorname{ker}\left(\nu_{1}^{ \pm}\right)$. The group $L^{ \pm}$has only one conjugacy class which is not contained in $K^{ \pm}$. This conjugacy class is the unique conjugacy class of involutions and has size $q \mp 1$.

For odd $q$ and for even $q$ we write $\Xi^{ \pm}$for the sum of all irreducible characters $\chi_{j}^{ \pm}$of $L^{ \pm}$of degree 2 .

Theorem 7.1. For odd $q$ and even $q$ the unipotent characters of $\mathrm{SO}_{5}(q)$ restricted to $P_{5}$ decompose as given in Table 5 .

REMARK 5 . The first column of Table 5 contains the symbols parameterizing the unipotent characters $\chi_{\Lambda}$ of $G=\mathrm{SO}_{5}(q)$. The second column lists symbols parameterizing characters $\sigma$ of $L$ such that ${ }^{1} \psi_{\sigma}$ is the Type 1 component of $\chi_{\Lambda} \downarrow_{P}^{G}$. The entries in columns 3-5 are characters $\vartheta$ such that ${ }^{\varepsilon} \psi_{\vartheta}$ is the Type $\varepsilon$ component of $\chi_{\Lambda \downarrow} \downarrow_{P}^{G}$.

TABle 5. Unipotent characters of $\mathrm{SO}_{5}(q)$ restricted to $P_{5}$.

\begin{tabular}{lllll}
\hline \multirow{1}{*}{$\Lambda$} & \multicolumn{4}{c}{$\chi_{\Lambda} \downarrow_{P}^{G}$} \\
\cline { 2 - 5 } & \multicolumn{1}{c}{ Type 1} & Type 0 & Type + & Type - \\
\hline$[2,-, 1]$ & {$[1,-, 1]$} & & \\
{$[-,-, 3]$} & & & & $\nu_{1}^{-}$ \\
{$\left[1^{2},-, 1\right]$} & {$[1,-, 1]$} & $1_{P_{3}}$ & & $1_{L^{-}}$ \\
{$[1,1,1]$} & {$[1,-, 1]+[-, 1,1]$} & $1_{P_{3}}$ & $1_{L^{+}}$ & \\
{$[-, 2,1]$} & {$[-, 1,1]$} & & $\nu_{1}^{+}$ & \\
{$\left[-, 1^{2}, 1\right]$} & {$[-, 1,1]$} & $1_{P_{3}}+\mu$ & $1_{L^{+}}+\nu_{1}^{+}\left(+\nu_{3}^{+}\right)+\Xi^{+}$ & $\left(\nu_{3}^{-}+\right) \Xi^{-}$ \\
\hline
\end{tabular}


The characters $\mu, \nu_{j}^{ \pm}$and $\Xi^{ \pm}$are defined in Remark 4. The characters in brackets in the last row of Table 5 only exist for odd $q$. More precisely: for odd prime powers $q$ the Type + component of $\chi_{\left[-, 1^{2}, 1\right]} \downarrow_{P}^{G}$ is ${ }^{+} \psi_{1_{L}++\nu_{1}^{+}+\nu_{3}^{+}+\Xi^{+}}$and the Type - component is ${ }^{-} \psi_{\nu_{3}^{-}+\Xi^{-}}$. For even prime powers $q$ the Type + component of $\chi_{\left[-, 1^{2}, 1\right]} \downarrow_{P}^{G}$ is ${ }^{+} \psi_{1_{L^{+}}+\nu_{1}^{+}+\Xi^{+}}$and the Type component is ${ }^{-} \psi_{\Xi^{-}}$.

Proof of Theorem 7.1. Let $\chi_{\Lambda}$ be a unipotent character of $G$. As in Remark 3, we write the restriction of $\chi_{\Lambda}$ to $P$ as $\chi_{\Lambda} \downarrow P={ }^{G} \chi+{ }^{0} \chi+{ }^{+} \chi+{ }^{-} \chi$, where ${ }^{\varepsilon} \chi={ }^{\varepsilon} \psi_{\vartheta \varepsilon}$ is the Type $\varepsilon$ component of $\chi_{\Lambda} \downarrow \stackrel{G}{P}$. The degrees $\vartheta^{\varepsilon}(1)$ can be computed from Table 4 via (6.4). The result is given in Table 6; it turns out that it does not depend on whether $q$ is odd or even.

The first row of Table 5 is trivial. Also, the first column of Table 5 can be determined from Table 6 and the combinatorics of bipartitions which encode Harish-Chandra induction and restriction; see $[\mathbf{9}, \S \S 3$ and 5$]$. In particular, we obtain

$$
\mathrm{R}_{L}^{G}\left(1_{L}\right)=\mathrm{R}_{L}^{G}\left(\chi_{[1,-, 1]}\right)=\chi_{[2,-, 1]}+\chi_{\left[1^{2},-, 1\right]}+\chi_{[1,1,1]} .
$$

By Proposition 5.1, we have $1_{L} \uparrow_{L}^{P}={ }^{1} \psi_{1_{L}}+{ }^{0} \psi_{1_{P_{3}}}+{ }^{+} \psi_{1_{L^{+}}}+{ }^{-} \psi_{1_{L^{-}}}$. Theorem 4.3(b) and the paragraph preceding it now yield for $\sigma=1_{L}$ and $\Sigma=1_{L}+\mathrm{St}_{L}$ the equation

$$
\mathrm{R}_{L}^{G}\left(1_{L}\right) \downarrow{ }_{P}^{G}=3 \cdot{ }^{1} \psi_{1_{L}}+{ }^{1} \psi_{\mathrm{St}_{L}}+2 \cdot{ }^{0} \psi_{1_{P_{3}}}+{ }^{+} \psi_{1_{L^{+}}}+{ }^{-} \psi_{1_{L^{-}}} .
$$

Rows 3 and 4 of Table 5 now follow from Table 6 . Next, we determine the restriction of the Steinberg character $\mathrm{St}_{G}=\chi_{\left[-, 1^{2}, 1\right]}$ to $P$. To apply Corollary 5.2, we have to describe the decomposition of $\mathrm{St}_{L^{\prime}} \downarrow_{P_{3}}^{L^{\prime}}$ and of $\mathrm{St}_{L} \downarrow_{L^{ \pm}}^{L}$ into irreducibles.

Since $\mathrm{St}_{L^{\prime}}$ vanishes on the non-trivial unipotent elements, the non-linear character $\mu \in$ $\operatorname{Irr}\left(P_{3}\right)$ of degree $q-1$ is a constituent of $\mathrm{St}_{L^{\prime}} \downarrow_{P_{3}}^{L^{\prime}}$. Since $1_{P_{3}} \uparrow P_{P_{3}}^{L^{\prime}}=1_{L^{\prime}}+\mathrm{St}_{L^{\prime}}$, Frobenius reciprocity implies that

$$
\mathrm{St}_{L^{\prime} \downarrow}{\stackrel{P_{3}}{\prime}}^{\prime}=1_{P_{3}}+\mu \text {. }
$$

Next, we consider the restriction of $\mathrm{St}_{L}$ to $L^{ \pm}$and $K^{ \pm}$, where $K^{ \pm}$is the cyclic normal subgroup of $L^{ \pm}$of index 2 which is defined in Remark 4(b). Suppose that $q$ is odd. By [3, Theorem 6.5.9], we have $\operatorname{St}_{L}(1)=q$ and $\operatorname{St}_{L}(\mathbf{x})=1$ for all $\mathbf{x} \in K^{+} \backslash\{1\}$. Thus, $\operatorname{St}_{L} \downarrow_{K^{+}}^{L}$ is the sum of the trivial character $1_{K^{+}}$and the regular character of $K^{+}$. It follows that exactly one of the characters $\nu_{2}^{+}, \nu_{3}^{+}$is a constituent of $\mathrm{St}_{L} \downarrow_{L^{+}}^{L}$ and that this constituent occurs with multiplicity one. By definition, this constituent is $\nu_{3}^{+}$, so that we get

$$
\begin{gathered}
\mathrm{St}_{L \downarrow} \downarrow_{L^{+}}^{L}=1_{L^{+}}+\nu_{1}^{+}+\nu_{3}^{+}+\Xi^{+} \quad \text { or } \quad \operatorname{St}_{L \downarrow} \downarrow_{L^{+}}^{L}=2 \cdot 1_{L^{+}}+\nu_{3}^{+}+\Xi^{+} \text {or } \\
\mathrm{St}_{L \downarrow_{L^{+}}^{L}}^{L}=2 \cdot \nu_{1}^{+}+\nu_{3}^{+}+\Xi^{+} .
\end{gathered}
$$

TABLE 6. Component degrees of the restrictions of the unipotent irreducible characters of $\mathrm{SO}_{5}(q)$ to $P_{5}$ for odd $q$ and even $q$.

\begin{tabular}{lllll}
\hline \multicolumn{1}{c}{$\Lambda$} & $\vartheta^{1}(1)$ & $\vartheta^{0}(1)$ & $\vartheta^{+}(1)$ & $\vartheta^{-}(1)$ \\
\hline$[2,-, 1]$ & 1 & $\cdot$ & $\cdot$ & $\cdot$ \\
{$[-,-, 3]$} & $\cdot$ & $\cdot$ & $\cdot$ & 1 \\
{$\left[1^{2},-, 1\right]$} & 1 & 1 & $\cdot$ & 1 \\
{$[1,1,1]$} & $q+1$ & 1 & 1 & $\cdot$ \\
{$[-, 2,1]$} & $q$ & $\cdot$ & 1 & $\cdot$ \\
{$\left[-, 1^{2}, 1\right]$} & $q$ & $q$ & $q$ & $q$ \\
\hline
\end{tabular}


Let $\mathbf{x}, \mathbf{x}^{\prime}$ be representatives for the two conjugacy classes of $L^{+}$which are not contained in $K^{+}$. Hence, $\mathbf{x}$ and $\mathbf{x}^{\prime}$ are involutions and, by [3, Theorem 6.5.9], we have $\operatorname{St}_{L}(\mathbf{x}), \operatorname{St}_{L}\left(\mathbf{x}^{\prime}\right) \in\{ \pm 1\}$. Thus,

$$
\left\langle 1_{L^{+}}, \mathrm{St}_{L} \downarrow_{L^{+}}^{L}\right\rangle_{L^{+}}=1+\frac{((q-1) / 2)\left(\mathrm{St}_{L}(\mathbf{x})+\mathrm{St}_{L}\left(\mathbf{x}^{\prime}\right)\right)}{2(q-1)}=1+\frac{1}{4}\left(\mathrm{St}_{L}(\mathbf{x})+\operatorname{St}_{L}\left(\mathbf{x}^{\prime}\right)\right) .
$$

It follows that $\left\langle 1_{L^{+}}, \mathrm{St}_{L} \downarrow_{L^{+}}^{L}\right\rangle_{L^{+}}=1$ and therefore $\mathrm{St}_{L \downarrow_{L^{+}}}=1_{L^{+}}+\nu_{1}^{+}+\nu_{3}^{+}+\Xi^{+}$. Next, we consider the restriction $\mathrm{St}_{L} \downarrow_{L^{-}}^{L}$ for odd $q$. By [3, Theorem 6.5.9], we have $\operatorname{St}_{L}(1)=q$ and $\mathrm{St}_{L}(\mathbf{x})=-1$ for all $\mathbf{x} \in K^{-} \backslash\{1\}$. Thus, $\mathrm{St}_{L} \downarrow_{K^{-}}^{L}$ is the regular character of $K^{-}$with the trivial character $1_{K^{-}}$removed. It follows that exactly one of $\nu_{2}^{-}, \nu_{3}^{-}$is a constituent of $\mathrm{St}_{L^{\prime}} \downarrow_{L^{-}}^{L}$ and that this constituent occurs with multiplicity one. By definition, this constituent is $\nu_{3}^{-}$ and thus $\mathrm{St}_{L \downarrow} \downarrow_{L^{-}}^{L}=\nu_{3}^{-}+\Xi^{-}$.

Now suppose that $q$ is even. As above, we see that $\mathrm{St}_{L} \downarrow_{K^{+}}^{L}$ is the sum of $1_{K^{+}}$and the regular character of $K^{+}$and that $\left\langle 1_{L^{+}}, \mathrm{St}_{L^{\prime}} \downarrow_{L^{+}}\right\rangle_{L^{+}}=1$. Hence, in this case we obtain that $\mathrm{St}_{L} \downarrow_{L^{+}}^{L}=1_{L^{+}}+\nu_{1}^{+}+\Xi^{+}$. As above, we see that $\mathrm{St}_{L} \downarrow_{K^{-}}^{L}$ is the regular character of $K^{-}$with the trivial character removed and get $\mathrm{St}_{L} \downarrow_{L^{-}}^{L}=\Xi^{-}$.

Applying Corollary 5.2, we get

$$
\mathrm{St}_{G \downarrow} \downarrow{ }_{P}^{G}=\chi_{\left[-, 1^{2}, 1\right]} \downarrow_{P}^{G}={ }^{1} \psi_{\mathrm{St}_{L}}+{ }^{0} \psi_{1_{P_{3}}+\mu}+{ }^{+} \psi_{1_{L^{+}}+\nu_{1}^{+}+\nu_{3}^{+}+\Xi^{+}}+{ }^{-} \psi_{\nu_{3}^{-}+\Xi^{-}}
$$

for odd $q$ and

$$
\mathrm{St}_{G \downarrow} \downarrow_{P}^{G}=\chi_{\left[-, 1^{2}, 1\right] \downarrow} \downarrow_{P}^{G}={ }^{1} \psi_{\mathrm{St}_{L}}+{ }^{0} \psi_{1_{P_{3}}+\mu}+{ }^{+} \psi_{1_{L^{+}}+\nu_{1}^{+}+\Xi^{+}}+{ }^{-} \psi_{\Xi^{-}}
$$

for even $q$, which proves the entries in row 6 of Table 5 . Next, we determine row 5 . We have

$$
\mathrm{R}_{L}^{G}\left(\mathrm{St}_{L}\right)=\mathrm{R}_{L}^{G}\left(\chi_{[-, 1,1]}\right)=\chi_{[1,1,1]}+\chi_{\left[-, 1^{2}, 1\right]}+\chi_{[-, 2,1]}
$$

and we want to determine $\mathrm{R}_{L}^{G}\left(\mathrm{St}_{L}\right) \downarrow_{P}^{G}$. Hence, we have to compute the summands on the righthand side of (4.8), where $\sigma=\mathrm{St}_{L}$. Since conjugation with $t$ permutes the unipotent characters of $L$, we have ${ }^{t} \sigma={ }^{t} \mathrm{St}_{L}=\mathrm{St}_{L}$. Hence, Theorem 4.3(a) and [3, Proposition 6.3.3] imply that

$$
{ }^{t} \sigma \downarrow_{L}^{t} P \uparrow_{L}^{P}=\mathrm{St}_{L} \uparrow_{L}^{P}=\mathrm{St}_{G \downarrow} \downarrow_{P}^{G}
$$

Suppressing symbols for inflation as in $\S 4.2$, we get from (7.1) that

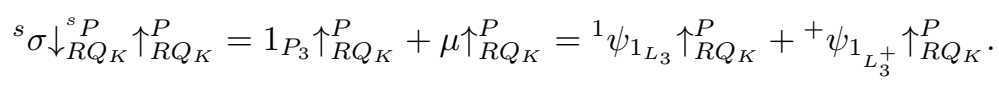

Note that $L_{3}^{+}=\{1\}$. From Theorem 4.3(b) and (d), we obtain

$$
\begin{gathered}
{ }^{1} \psi_{1_{L_{3}} \uparrow R Q_{K}}={ }^{0} \psi_{1_{P_{3}}}+{ }^{1} \psi_{\mathrm{R}_{L_{3}}^{L_{5}}\left(1_{L_{3}}\right)}={ }^{0} \psi_{1_{P_{3}}}+{ }^{1} \psi_{1_{L}}+{ }^{1} \psi_{\mathrm{St}_{L}}, \\
{ }^{+} \psi_{1_{L_{3}^{+}} \uparrow}{ }_{R Q_{K}}^{P}={ }^{+} \psi_{\mathrm{R}_{L_{3}^{+}}^{L^{+}}\left(1_{L_{3}^{+}}\right)}={ }^{+} \psi_{1_{L^{+}}}+{ }^{+} \psi_{\nu_{1}^{+}} .
\end{gathered}
$$

Thus, we get from (4.8) and (7.4)-(7.7) that

$$
\begin{aligned}
\mathrm{R}_{L}^{G}\left(\mathrm{St}_{L}\right) \downarrow_{P}^{G} & ={ }^{1} \psi_{\mathrm{St}_{L}}+{ }^{0} \psi_{1_{P_{3}}}+{ }^{1} \psi_{1_{L}}+{ }^{1} \psi_{\mathrm{St}_{L}}+{ }^{+} \psi_{1_{L^{+}}}+{ }^{+} \psi_{\nu_{1}^{+}}+\mathrm{St}_{G} \downarrow_{P}^{G} \\
& =\chi_{[1,1,1] \downarrow} \downarrow_{P}^{G}+\chi_{[-, 2,1]} \downarrow_{P}^{G}+\mathrm{St}_{G \downarrow} \downarrow_{P}^{G} .
\end{aligned}
$$

Since we already know $\chi_{[1,1,1]} \downarrow{ }_{P}^{G}$, we get $\chi_{[-, 2,1]} \downarrow{ }_{P}^{G}={ }^{1} \psi_{\mathrm{St}_{L}}+{ }^{+} \psi_{\nu_{1}^{+}}$, completing row 5 of Table 5. 
Finally, we consider $\chi_{[-,-, 3]} \downarrow_{P}^{G}$. We know from Table 6 that $\chi_{[-,-, 3]} \downarrow_{P}^{G}={ }^{-} \psi_{\vartheta}$ for a linear character $\vartheta \in \operatorname{Irr}\left(L^{-}\right)$. Therefore, from the definition of ${ }^{-} \psi_{\vartheta}$, it follows that

$$
\chi_{[-,-, 3]} \downarrow_{L^{-}}^{G}={ }^{-} \psi_{\vartheta} \downarrow_{L^{-}}^{P}=\vartheta \uparrow_{L^{-}}^{L} \downarrow_{L^{-}}^{L} .
$$

Suppose that $q$ is odd. It follows from Remark 4(b) that $L^{-}$has exactly three conjugacy classes of involutions and we choose representatives $\tau_{1}, \tau_{2}$ and $\tau_{3}$ for these classes such that $\tau_{1}, \tau_{2} \notin K^{-}$ and $\tau_{3} \in Z\left(L^{-}\right)$. Let $c_{j}^{L}$ be the $L$-conjugacy class of $\tau_{j}$ for $j=1,2,3$. We have already seen that $\mathrm{St}_{L} \downarrow_{L^{-}}^{L}=\nu_{3}^{-}+\Xi^{-}$. Since $\Xi^{-}$vanishes on $L^{-} \backslash K^{-}$, we have $\operatorname{St}_{L}\left(\tau_{1}\right)=\nu_{3}^{-}\left(\tau_{1}\right) \neq \nu_{3}^{-}\left(\tau_{2}\right)=$ $\mathrm{St}_{L}\left(\tau_{2}\right)$; hence, $c_{1}^{L} \neq c_{2}^{L}$. Also, from Remark 4(b), we get that $c_{3}^{L} \subseteq L^{\prime} \unlhd L$ and $c_{1}^{L}, c_{2}^{L} \nsubseteq L^{\prime}$. Thus, $c_{1}^{L}, c_{2}^{L}, c_{3}^{L}$ are pairwise distinct. Since the conjugacy classes of $L=A \times L^{\prime} \cong \mathbb{F}_{q}^{\times} \times \mathrm{SO}_{3}(q)$ are known, we see that $\left|C_{L}\left(\tau_{1}\right)\right|,\left|C_{L}\left(\tau_{2}\right)\right| \in\left\{2\left(q^{2}-1\right), 2(q-1)^{2}\right\}$. Hence,

$$
\chi_{[-,-, 3]}\left(\tau_{j}\right)=\vartheta \uparrow_{L^{-}}^{L}\left(\tau_{j}\right) \in\left\{\frac{q^{2}-1}{2} \vartheta\left(\tau_{j}\right), \frac{(q-1)^{2}}{2} \vartheta\left(\tau_{j}\right)\right\}
$$

for $j=1,2$. From the character values of $\chi_{[-,-, 3]}$, we get $\vartheta\left(\tau_{1}\right)=\vartheta\left(\tau_{2}\right)=-1$ and hence $\vartheta=\nu_{1}^{-}$.

Suppose that $q$ is even. There is just one conjugacy class of involutions in $L^{-}$; let $\tau$ be a representative for this class. The group $L \cong \mathbb{F}_{q}^{\times} \times \mathrm{SO}_{3}(q) \cong \mathbb{F}_{q}^{\times} \times \mathrm{SL}_{2}(q)$ has only one conjugacy class of involutions and we see that $\left|C_{L}(\tau)\right|=q(q-1)$. Hence, we compute $\chi_{[-,-, 3]}(\tau)=$ $\vartheta \uparrow_{L^{-}}^{L}(\tau)=\frac{1}{2} q(q-1) \vartheta(\tau)$. From the character table of $\operatorname{SO}_{5}(q) \cong \operatorname{Sp}_{4}(q)$ in CHEVIE, we get $\chi_{[-,-, 3]}(\tau)=-\frac{1}{2} q(q-1)$; thus, $\vartheta=\nu_{1}^{-}$. This completes the proof of Theorem 7.1.

\section{Restrictions of unipotent characters of $\mathrm{SO}_{7}(q)$}

In this section we obtain some information on the decomposition into irreducible characters of the restrictions of the unipotent characters of $G_{7}=\mathrm{SO}_{7}(q)$ to the parabolic subgroup $P_{7}$. In the whole section we assume that $m=3$ and $n=2 m+1=7$. We set $G:=\mathrm{SO}_{7}(q), P:=P_{7}$, $L:=L_{7}$ and $L^{ \pm}:=L_{7}^{ \pm}$for brevity. The degrees of the unipotent characters of $\mathrm{SO}_{7}(q)$ and their labels are given in Table 7 . We use the abbreviations $\phi_{1}:=q-1, \phi_{2}:=q+1, \phi_{3}:=q^{2}+q+1$, $\phi_{4}:=q^{2}+1$ and $\phi_{6}:=q^{2}-q+1$.

As in $\S 6$, we denote the $P$-conjugacy class of the unipotent element $\mathbf{z}_{j}$ by $c_{j}$ for $j=0,1,2$. The conjugacy classes and the values of the unipotent characters of $G=\mathrm{SO}_{7}(q)$ were computed

TABLE 7. Labels and degrees of the unipotent characters of $\mathrm{SO}_{7}(q)$.

\begin{tabular}{llllll}
\hline Bipartition & Symbol & Degree & Bipartition & Symbol & Degree \\
\hline$[3,-, 1]$ & $\left(\begin{array}{c}3 \\
-\end{array}\right)$ & 1 & {$\left[1^{2}, 1,1\right]$} & $\left(\begin{array}{c}12 \\
1\end{array}\right)$ & $q^{3} \phi_{3} \phi_{6}$ \\
{$[2,1,1]$} & $\left(\begin{array}{c}03 \\
1\end{array}\right)$ & $\frac{1}{2} q \phi_{3} \phi_{4}$ & {$\left[1,1^{2}, 1\right]$} & $\left(\begin{array}{c}013 \\
12\end{array}\right)$ & $\frac{1}{2} q^{4} \phi_{3} \phi_{4}$ \\
{$[-, 3,1]$} & $\left(\begin{array}{c}01 \\
3\end{array}\right)$ & $\frac{1}{2} q \phi_{4} \phi_{6}$ & {$[-, 21,1]$} & $\left(\begin{array}{c}012 \\
13\end{array}\right)$ & $\frac{1}{2} q^{4} \phi_{2}^{2} \phi_{6}$ \\
{$[21,-, 1]$} & $\left(\begin{array}{c}13 \\
0\end{array}\right)$ & $\frac{1}{2} q \phi_{2}^{2} \phi_{6}$ & {$\left[1^{3},-, 1\right]$} & $\left(\begin{array}{c}123 \\
01\end{array}\right)$ & $\frac{1}{2} q^{4} \phi_{4} \phi_{6}$ \\
{$[1,-, 3]$} & $\left(\begin{array}{c}013 \\
-\end{array}\right)$ & $\frac{1}{2} q \phi_{1}^{2} \phi_{3}$ & {$[-, 1,3]$} & $\left(\begin{array}{c}0123 \\
1\end{array}\right)$ & $\frac{1}{2} q^{4} \phi_{1}^{2} \phi_{3}$ \\
{$[1,2,1]$} & $\left(\begin{array}{c}02 \\
2\end{array}\right)$ & $q^{2} \phi_{3} \phi_{6}$ & {$\left[-, 1^{3}, 1\right]$} & $\left(\begin{array}{c}0123 \\
123\end{array}\right)$ & $q^{9}$ \\
\hline
\end{tabular}


by Frank Lübeck (private communication, 2010). For odd $q$ the group $G$ has 10 unipotent conjugacy classes and for even $q$ the group $G$ has 12 unipotent classes. Remark 2 and the centralizer orders (6.1) determine the fusion of the classes $c_{0}, c_{1}, c_{2}$ into the unipotent classes of $G$ uniquely so that we can read off the values of the unipotent characters of $G$ on the elements $\mathbf{z}_{0}, \mathbf{z}_{1}, \mathbf{z}_{2}$ in Table 8 from Lübeck's data. In the table, zeros are replaced by dots.

Additionally, we have the values $\chi_{[3,-, 1]}\left(\mathbf{z}_{j}\right)=1$ for all $j$ for the trivial character and $\chi_{\left[-, 1^{3}, 1\right]}\left(\mathbf{z}_{j}\right)=0$ for all $j$ for the Steinberg character.

Theorem 8.1. For odd $q$ and even $q$ the Type 1 and Type 0 components of the restrictions of the unipotent characters of $\mathrm{SO}_{7}(q)$ to $P_{7}$ are given in Table 9.

TABLE 8. Values $\chi_{\Lambda}\left(\mathbf{z}_{j}\right)$ of unipotent characters of $\mathrm{SO}_{7}(q)$.

\begin{tabular}{|c|c|c|c|c|c|c|}
\hline \multirow[b]{2}{*}{$\Lambda$} & \multicolumn{3}{|c|}{$q$ odd } & \multicolumn{3}{|c|}{$q$ even } \\
\hline & $\mathbf{z}_{0}$ & $\mathbf{z}_{1}$ & $\mathbf{z}_{2}$ & $\mathbf{z}_{0}$ & $\mathbf{z}_{1}$ & $\mathbf{z}_{2}$ \\
\hline$[2,1,1]$ & $\frac{1}{2} q\left(2 q^{2}+q+1\right)$ & $\frac{1}{2} q \phi_{2}^{2}$ & $\frac{1}{2} q \phi_{4}$ & $\frac{1}{2} q\left(2 q^{2}+q+1\right)$ & $\frac{1}{2} q \phi_{2} \phi_{4}$ & $\frac{1}{2} q \phi_{3}$ \\
\hline$[-, 3,1]$ & $\frac{1}{2} q\left(2 q^{2}-q+1\right)$ & $\frac{1}{2} q \phi_{1}^{2}$ & $\frac{1}{2} q \phi_{4}$ & $\frac{1}{2} q\left(2 q^{2}-q+1\right)$ & $-\frac{1}{2} q \phi_{1} \phi_{4}$ & $\frac{1}{2} q \phi_{6}$ \\
\hline$[21,-, 1]$ & $\frac{1}{2} q \phi_{2}$ & $\frac{1}{2} q \phi_{2}^{2}$ & $\frac{1}{2} q \phi_{4}$ & $\frac{1}{2} q \phi_{2}$ & $\frac{1}{2} q \phi_{2} \phi_{4}$ & $\frac{1}{2} q \phi_{3}$ \\
\hline$[1,-, 3]$ & $-\frac{1}{2} q \phi_{1}$ & $\frac{1}{2} q \phi_{1}^{2}$ & $\frac{1}{2} q \phi_{4}$ & $-\frac{1}{2} q \phi_{1}$ & $-\frac{1}{2} q \phi_{1} \phi_{4}$ & $\frac{1}{2} q \phi_{6}$ \\
\hline$[1,2,1]$ & $q^{2} \phi_{4}$ & $q^{2}$ & $q^{2}$ & $q^{2} \phi_{4}$ & $q^{2}$ & $q^{2}$ \\
\hline$\left[1^{2}, 1,1\right]$ & $q^{3}$ & $2 q^{3}$ & . & $q^{3}$ & $q^{3} \phi_{4}$ & $q^{3}$ \\
\hline$\left[1,1^{2}, 1\right]$ & $\frac{1}{2} q^{4} \phi_{2}$ & $q^{4}$ & . & $\frac{1}{2} q^{4} \phi_{2}$ & $\frac{1}{2} q^{4} \phi_{4}$ & $\frac{1}{2} q^{4}$ \\
\hline$[-, 21,1]$ & $\frac{1}{2} q^{4} \phi_{2}$ & & $q^{4}$ & $\frac{1}{2} q^{4} \phi_{2}$ & $-\frac{1}{2} q^{4} \phi_{1} \phi_{2}$ & $\frac{1}{2} q^{4}$ \\
\hline$\left[1^{3},-, 1\right]$ & $-\frac{1}{2} q^{4} \phi_{1}$ & $q^{4}$ & . & $-\frac{1}{2} q^{4} \phi_{1}$ & $\frac{1}{2} q^{4} \phi_{4}$ & $\frac{1}{2} q^{4}$ \\
\hline$[-, 1,3]$ & $-\frac{1}{2} q^{4} \phi_{1}$ & & $q^{4}$ & $-\frac{1}{2} q^{4} \phi_{1}$ & $-\frac{1}{2} q^{4} \phi_{1} \phi_{2}$ & $\frac{1}{2} q^{4}$ \\
\hline
\end{tabular}

TABLE 9. Type 1 and Type 0 components of the restrictions of the unipotent characters of $\mathrm{SO}_{7}(q)$ to the parabolic subgroup $P_{7}$.

\begin{tabular}{|c|c|c|c|c|c|}
\hline \multirow[b]{3}{*}{$\Lambda$} & \multicolumn{5}{|c|}{$\chi_{\Lambda} \downarrow_{P}^{G}$} \\
\hline & \multirow[b]{2}{*}{ Type 1} & \multicolumn{4}{|c|}{ Type 0} \\
\hline & & 1 & 0 & + & - \\
\hline$[3,-, 1]$ & {$[2,-, 1]$} & & & & \\
\hline$[2,1,1]$ & {$[2,-, 1]+[1,1,1]$} & {$[1,-, 1]$} & & & \\
\hline$[-, 3,1]$ & {$[-, 2,1]$} & & & & \\
\hline$[21,-, 1]$ & {$[2,-, 1]+\left[1^{2},-, 1\right]$} & {$[1,-, 1]$} & & & \\
\hline$[1,-, 3]$ & {$[-,-, 3]$} & & & & \\
\hline $\begin{array}{l}{[1,2,1]} \\
{\left[1^{2}, 1,1\right]}\end{array}$ & $\begin{array}{l}{[1,1,1]+[-, 2,1]} \\
{\left[1^{2},-, 1\right]+[1,1,1]}\end{array}$ & $\begin{array}{l}{[-, 1,1]} \\
{[1,-, 1]+[-, 1,1]}\end{array}$ & $1_{P_{3}}$ & & \\
\hline$\left[1,1^{2}, 1\right]$ & {$\left[-, 1^{2}, 1\right]+[1,1,1]$} & {$[1,-, 1]+[-, 1,1]$} & $1_{P_{3}}$ & $1_{L_{5}^{+}}$ & \\
\hline$[-, 21,1]$ & {$\left[-, 1^{2}, 1\right]+[-, 2,1]$} & {$[-, 1,1]$} & & $\nu_{1}^{+}$ & \\
\hline$\left[1^{3},-, 1\right]$ & {$\left[1^{2},-, 1\right]$} & {$[1,-, 1]$} & $1_{P_{3}}$ & & $1_{L_{5}^{-}}$ \\
\hline$[-, 1,3]$ & {$[-,-, 3]$} & & & & $\nu_{1}^{-}$ \\
\hline$\left[-, 1^{3}, 1\right]$ & {$\left[-, 1^{2}, 1\right]$} & {$[-, 1,1]$} & $1_{P_{3}}+\mu$ & $1_{L_{5}^{+}}+\nu_{1}^{+}\left(+\nu_{3}^{+}\right)+\Xi^{+}$ & $\left(\nu_{3}^{-}+\right) \Xi^{-}$ \\
\hline
\end{tabular}


REMARK 6 . The first column of Table 9 contains the symbols parameterizing the unipotent characters $\chi_{\Lambda}$ of $G=\mathrm{SO}_{7}(q)$. The second column lists symbols parameterizing characters $\sigma$ of $L$ such that ${ }^{1} \psi_{\sigma}$ is the Type 1 component of $\chi_{\Lambda} \downarrow_{P}^{G}$. The entries in columns 3-6 are characters $\rho^{1}, \rho^{0}, \rho^{+}, \rho^{-}$, respectively, such that ${ }^{0} \psi_{\left({ }^{1} \psi_{\rho^{1}}\right)}+{ }^{0} \psi_{\left({ }^{0} \psi_{\rho^{0}}\right)}+{ }^{0} \psi_{\left({ }^{+} \psi_{\rho^{+}}\right)}+{ }^{0} \psi_{\left(-\psi_{\rho^{-}}\right)}$is the Type 0 component of $\chi_{\Lambda} \downarrow_{P}^{G}$.

The characters $\mu, \nu_{j}^{ \pm}$and $\Xi^{ \pm}$are defined in Remark 4 . The characters in brackets in the last row of Table 9 only exist for odd $q$. More precisely: the Type 0 component of $\chi_{\left[-, 1^{3}, 1\right]} \downarrow P$ is ${ }^{0} \psi_{\Gamma}$, where $\Gamma:={ }^{1} \psi_{\chi_{[-, 1,1]}}+{ }^{0} \psi_{1_{P_{3}}}+{ }^{0} \psi_{\mu}+{ }^{+} \psi_{1_{L_{5}^{+}}}+{ }^{+} \psi_{\nu_{1}^{+}}+{ }^{+} \psi_{\nu_{3}^{+}}+{ }^{+} \psi_{\Xi^{+}}+{ }^{-} \psi_{\nu_{3}^{-}}+{ }^{-} \psi_{\Xi^{-}}$ for odd $q$ and $\Gamma:={ }^{1} \psi_{\chi_{[-, 1,1]}}+{ }^{0} \psi_{1_{P_{3}}}+{ }^{0} \psi_{\mu}+{ }^{+} \psi_{1_{L_{5}^{+}}}+{ }^{+} \psi_{\nu_{1}^{+}}+{ }^{+} \psi_{\Xi^{+}}+{ }^{-} \psi_{\Xi^{-}}$for even $q$.

Proof of Theorem 8.1. Let $\chi_{\Lambda}$ be a unipotent character of $G$. As in Remark 3, we write the restriction of $\chi_{\Lambda}$ to $P$ as $\chi_{\Lambda} \downarrow P={ }_{P}^{G} \chi+{ }^{0} \chi+{ }^{+} \chi+{ }^{-} \chi$, where ${ }^{\varepsilon} \chi={ }^{\varepsilon} \psi_{\vartheta \varepsilon}$ is the Type $\varepsilon$ component of $\chi_{\Lambda} \downarrow_{P}^{G}$. The degrees $\vartheta^{\varepsilon}(1)$ can be computed from Table 8 via (6.4). The result is given in Table 10; it turns out that it does not depend on whether $q$ is odd or even.

The first row of Table 9 is trivial and rows 3 and 5 follow from Table 10. Also, the first column of Table 9 can be determined from Table 10 and the combinatorics of bipartitions which encode Harish-Chandra induction and restriction. In particular, we obtain

$$
\mathrm{R}_{L}^{G}\left(1_{L}\right)=\mathrm{R}_{L}^{G}\left(\chi_{[2,-, 1]}\right)=\chi_{[3,-, 1]}+\chi_{[2,1,1]}+\chi_{[21,-, 1]} .
$$

By Proposition 5.1, we have $1_{L} \uparrow \uparrow_{L}^{P}={ }^{1} \psi_{1_{L}}+{ }^{0} \psi_{1_{P_{5}}}+{ }^{+} \psi_{1_{L^{+}}}+{ }^{-} \psi_{1_{L^{-}}}$. Theorem 4.3(b) and the paragraph preceding it now yield for $\sigma=1_{L}, \Sigma=\chi_{[2,-, 1]}+\chi_{\left[1^{2},-, 1\right]}+\chi_{[1,1,1]}$ the equation

$$
\begin{aligned}
\mathrm{R}_{L}^{G}\left(1_{L}\right) \downarrow_{P}^{G} & =1_{P}+1_{R Q_{K}} \uparrow_{R Q_{K}}^{P}+1_{L} \uparrow_{L}^{P} \\
& =1_{P}+{ }^{0}{\psi_{1_{P_{5}}}}^{1}{ }^{1} \psi_{\Sigma}+{ }^{1} \psi_{1_{L}}+{ }^{0} \psi_{1_{P_{5}}}+{ }^{+} \psi_{1_{L^{+}}}+{ }^{-} \psi_{1_{L^{-}}} .
\end{aligned}
$$

Now the entries in rows 2 and 4 follow from Table 10 . We set $\eta:=\chi_{[-,-, 3]} \in \operatorname{Irr}(L)$. We have

$$
\mathrm{R}_{L}^{G}(\eta)=\mathrm{R}_{L}^{G}\left(\chi_{[-,-, 3]}\right)=\chi_{[1,-, 3]}+\chi_{[-, 1,3]} .
$$

Since conjugation with $t$ permutes the unipotent characters of $L$ and since $\eta$ is the unique non-

\begin{tabular}{|c|c|c|c|c|}
\hline$\Lambda$ & $\vartheta^{1}(1)$ & $\vartheta^{0}(1)$ & $\vartheta^{+}(1)$ & $\vartheta^{-}(1)$ \\
\hline$[3,-, 1]$ & 1 & & . & . \\
\hline$[2,1,1]$ & $\frac{1}{2}(q+2)\left(q^{2}+1\right)$ & 1 & 1 & . \\
\hline$[-, 3,1]$ & $\frac{1}{2} q\left(q^{2}+1\right)$ & . & 1 & . \\
\hline$[21,-, 1]$ & $\frac{1}{2}(q+1)\left(q^{2}-q+2\right)$ & 1 & . & 1 \\
\hline$[1,-, 3]$ & $\frac{1}{2} q(q-1)^{2}$ & . & . & 1 \\
\hline$[1,2,1]$ & $q\left(q^{2}+q+1\right)$ & $q$ & $2 q$ & . \\
\hline$\left[1^{2}, 1,1\right]$ & $q\left(q^{2}+q+1\right)$ & $q(q+1)$ & $q^{2}$ & $q^{2}$ \\
\hline$\left[1,1^{2}, 1\right]$ & $\frac{1}{2} q(2 q+1)\left(q^{2}+1\right)$ & $\frac{1}{2} q(q+1)^{2}$ & $\frac{1}{2} q(q+1)^{2}$ & $\frac{1}{2} q\left(q^{2}+1\right)$ \\
\hline$[-, 21,1]$ & $\frac{1}{2} q\left(q^{2}+2 q^{3}+1\right)$ & $\frac{1}{2} q\left(q^{2}+1\right)$ & $\frac{1}{2} q(q+1)^{2}$ & $\frac{1}{2} q\left(q^{2}+1\right)$ \\
\hline$\left[1^{3},-, 1\right]$ & $\frac{1}{2} q\left(q^{2}+1\right)$ & $\frac{1}{2} q\left(q^{2}+1\right)$ & $\frac{1}{2} q(q-1)^{2}$ & $\frac{1}{2} q\left(q^{2}+1\right)$ \\
\hline$[-, 1,3]$ & $\frac{1}{2} q(q-1)^{2}$ & $\frac{1}{2} q(q-1)^{2}$ & $\frac{1}{2} q(q-1)^{2}$ & $\frac{1}{2} q\left(q^{2}+1\right)$ \\
\hline$\left[-, 1^{3}, 1\right]$ & $q^{4}$ & $q^{4}$ & $q^{4}$ & $q^{4}$ \\
\hline
\end{tabular}
trivial unipotent character of $L$ of degree $\frac{1}{2} q(q-1)^{2}$, we have ${ }^{t} \eta=\eta$. Hence, we get from (4.8)

TABLE 10. Component degrees of the restrictions of the unipotent irreducible characters of $\mathrm{SO}_{7}(q)$ to $P_{7}$ for both odd $q$ and even $q$. 
that

$$
\mathrm{R}_{L}^{G}(\eta) \downarrow_{P}^{G}={ }^{1} \psi_{\eta}+{ }^{s} \eta \downarrow_{R Q_{K}}^{s} \uparrow_{R Q_{K}}^{P}+\eta \uparrow_{L}^{P} .
$$

By Proposition 5.1 and Theorem 7.1, the character ${ }^{0} \psi_{\left(-\psi_{\nu_{1}^{-}}\right.}$is a constituent of $\eta_{\uparrow_{L}}^{P}$. From (8.1), we get $\mathrm{R}_{L}^{G}(\eta) \downarrow_{P}^{G}=\chi_{[1,-, 3]} \downarrow_{P}^{G}+\chi_{[-, 1,3]} \downarrow_{P}^{G}$ and, since $\chi_{[1,-, 3]} \downarrow_{P}^{G}$ does not have any Type 0 component, we see that ${ }^{0} \psi_{\left(-\psi_{\nu_{1}^{-}}\right.}$is a constituent of $\chi_{[-, 1,3]} \downarrow_{P}^{G}$. From the degree ${ }^{-} \psi_{\nu_{1}^{-}}(1)=$ $\frac{1}{2} q(q-1)^{2}$ and Table 10, we can conclude that ${ }^{0} \psi_{\left(-\psi_{\nu_{1}^{-}}\right)}$is the Type 0 component of $\chi_{[-, 1,3]} \downarrow_{P}^{G}$, proving the entries in row 11 of Table 9 . We have

$$
\mathrm{R}_{L}^{G}\left(\chi_{[-, 2,1]}\right)=\chi_{[-, 3,1]}+\chi_{[1,2,1]}+\chi_{[-, 21,1]} .
$$

Conjugation with $t$ permutes the unipotent characters of $L$. The characters $\chi_{[-, 2,1]}$ and $\chi_{\left[1^{2},-, 1\right]}$ are the only unipotent characters of $L$ of degree $\frac{1}{2} q\left(q^{2}+1\right)$. Since the conjugacy class of $L$ containing $\mathbf{z}_{0}$ is fixed by conjugation with $t$ and since the values of $\chi_{[-, 2,1]}$ and $\chi_{\left[1^{2},-, 1\right]}$ on this class differ, we have ${ }^{t} \chi_{[-, 2,1]}=\chi_{[-, 2,1]}$ and ${ }^{t} \chi_{\left[1^{2},-, 1\right]}=\chi_{\left[1^{2},-, 1\right]}$. Hence, we get from (4.8) that

$$
\mathrm{R}_{L}^{G}\left(\chi_{[-, 2,1]}\right) \downarrow_{P}^{G}={ }^{1} \psi_{\chi_{[-, 2,1]}}+{ }^{s} \chi_{[-, 2,1]} \downarrow_{R Q_{K}}^{s} \uparrow_{R Q_{K}}^{P}+\chi_{[-, 2,1]} \uparrow_{L}^{P} .
$$

By Theorem 7.1 and Theorem 4.3(b), the character ${ }^{0} \psi_{1} \psi_{\chi_{[-, 1,1]}}$ is a constituent of the character ${ }^{s} \chi_{[-, 2,1]} \downarrow_{R Q_{K}}^{s} \uparrow_{R Q_{K}}^{P}$. Furthermore, it follows from Theorem 7.1 and Proposition 5.1 that ${ }^{0} \psi_{1} \psi_{\chi_{[-, 1,1]}}+{ }^{0} \psi_{\left({ }^{+} \psi_{\nu_{1}^{+}}\right)}$is a subcharacter of $\chi_{[-, 2,1]} \uparrow_{L}^{P}$. Thus, (8.2) implies that $2 \cdot{ }^{0} \psi_{1} \psi_{\chi_{[-, 1,1]}}+$ ${ }^{0} \psi_{\left({ }^{+} \psi_{\nu_{1}^{+}}\right.}$is a subcharacter of $\chi_{[-, 3,1]} \downarrow_{P}^{G}+\chi_{[1,2,1]} \downarrow_{P}^{G}+\chi_{[-, 21,1]} \downarrow_{P}^{G}$. Since $\chi_{[-, 3,1]} \downarrow_{P}^{G}$ does not have any Type 0 component, we see that $2 \cdot{ }^{0} \psi_{1} \psi_{\chi_{[-, 1,1]}}+{ }^{0} \psi_{\left({ }^{+} \psi_{\nu_{1}^{+}}\right)}$is a subcharacter of the restriction $\chi_{[1,2,1]} \downarrow_{P}^{G}+\chi_{[-, 21,1]} \downarrow_{P}^{G}$. Because ${ }^{1} \psi_{\chi_{[-, 1,1]}}(1)=q$ and ${ }^{+} \psi_{\nu_{1}^{+}}(1)=\frac{1}{2} q\left(q^{2}-1\right)$, we get from Table 10 that ${ }^{0} \psi_{1} \psi_{\chi_{[-, 1,1]}}$ is the Type 0 component of $\chi_{[1,2,1]} \downarrow{ }_{P}^{G}$ and ${ }^{0} \psi_{1} \psi_{\chi_{[-, 1,1]}}+{ }^{0} \psi_{\left({ }^{+} \psi_{\nu_{1}^{+}}\right)}$ is the Type 0 component of $\chi_{[-, 21,1]} \downarrow_{P}^{G}$. This proves the entries in rows 6 and 9 of Table 9.

From Corollary 5.2 and Theorem 7.1, we get that the Type 0 component of the restriction $\chi_{\left[-, 1^{3}, 1\right]} \downarrow_{P}^{G}=\mathrm{St}_{G} \downarrow_{P}^{G}$ is

$$
\begin{aligned}
& { }^{0} \psi_{\left({ }^{1} \psi_{\chi_{[-, 1,1]}}\right)}+{ }^{0} \psi_{\left({ }^{0} \psi_{1_{P_{3}}}\right)}+{ }^{0} \psi_{\left({ }^{0} \psi_{\mu}\right)}+{ }^{0} \psi_{\left({ }^{+} \psi_{1_{L_{5}^{+}}}\right)}+{ }^{0} \psi_{\left({ }^{+} \psi_{\nu_{1}^{+}}\right)}\left(+{ }^{0} \psi_{\left(+\psi_{\nu_{3}^{+}}\right)}\right) \\
& \quad+{ }^{0} \psi_{\left({ }^{+} \psi_{\Xi^{+}}\right)}\left(+{ }^{0} \psi_{\left(-\psi_{\nu_{3}^{-}}\right)}\right)+{ }^{0} \psi_{\left(-\psi_{\Xi^{-}}\right)}
\end{aligned}
$$

where the summands in brackets only occur for odd $q$. This proves the entries in row 12 of Table 9. We have

$$
\mathrm{R}_{L}^{G}\left(\chi_{\left[-, 1^{2}, 1\right]}\right)=\chi_{\left[1,1^{2}, 1\right]}+\chi_{[-, 21,1]}+\chi_{\left[-, 1^{3}, 1\right]} .
$$

Since conjugation with $t$ permutes the unipotent characters of $L$, we have ${ }^{t} \chi_{\left[-, 1^{2}, 1\right]}={ }^{t} \mathrm{St}_{L}=$ $\chi_{\left[-, 1^{2}, 1\right]}$. Hence, we get from (4.8) and [3, Proposition 6.3.3] that

$$
\begin{aligned}
\mathrm{R}_{L}^{G}\left(\chi_{\left[-, 1^{2}, 1\right]}\right) \downarrow_{P}^{G} & ={ }^{1} \psi_{\chi_{\left[-, 1^{2}, 1\right]}}+{ }^{s} \chi_{\left[-, 1^{2}, 1\right]} \downarrow_{R Q_{K}} \uparrow_{R Q_{K}}+\chi_{\left[-, 1^{2}, 1\right]} \uparrow_{L}^{P} \\
& ={ }^{1} \psi_{\chi_{\left[-, 1^{2}, 1\right]}}+{ }^{s} \chi_{\left[-, 1^{2}, 1\right]} \downarrow_{R Q_{K}} \uparrow_{R Q_{K}}+\chi_{\left[-, 1^{3}, 1\right]} \downarrow_{P}^{G} .
\end{aligned}
$$

Together with (8.3), this implies that the Type 0 component of $\chi_{\left[1,1^{2}, 1\right]} \downarrow_{P}^{G}+\chi_{[-, 21,1]} \downarrow_{P}^{G}$ is the Type 0 component of ${ }^{s} \chi_{\left[-, 1^{2}, 1\right]} \downarrow_{R Q_{K}}^{s} \uparrow_{R Q_{K}}^{P}$. We obtain from Theorems 7.1 and $4.3(\mathrm{~b})$ and (c) that ${ }^{0} \psi_{\left({ }^{1} \psi_{\chi_{[-, 1,1]}}\right)}+{ }^{0} \psi_{\Sigma}$ is a subcharacter of ${ }^{s} \chi_{\left[-, 1^{2}, 1\right]} \downarrow_{R Q_{K}}^{s_{P}} \uparrow_{R Q_{K}}^{P}$, where $\Sigma:=1_{r} P_{5} \cap P_{5} \uparrow_{r} P_{P_{5}}^{P_{5}} P_{5}$. The permutation character $\Sigma$ can be computed via Theorem $4.3(\mathrm{~b})$ and we get $\Sigma={ }^{0} \psi_{1_{P_{3}}}+$ ${ }^{1} \psi_{1_{L_{5}}}+{ }^{1} \psi_{\mathrm{St}_{L_{5}}}$, so that

$$
{ }^{0} \psi_{\left({ }^{1} \psi_{\chi_{[-, 1,1]}}\right)}+{ }^{0} \psi_{\left({ }^{0} \psi_{1_{P_{3}}}\right)}+{ }^{0} \psi_{\left({ }^{1} \psi_{\chi_{[1,-, 1]}}\right)}+{ }^{0} \psi_{\left({ }^{1} \psi_{\chi_{[-, 1,1]}}\right)}
$$


is a subcharacter of the Type 0 component of $\chi_{\left[1,1^{2}, 1\right]} \downarrow_{P}^{G}+\chi_{[-, 21,1]} \downarrow_{P}^{G}$. Because we already know the Type 0 component of $\chi_{[-, 21,1]} \downarrow_{P}^{G}$, we see that

$$
{ }^{0} \psi_{\left({ }^{0} \psi_{P_{3}}\right)}+{ }^{0} \psi_{\left({ }^{1} \psi_{\chi_{[1,-, 1]}}\right)}+{ }^{0} \psi_{\left({ }^{1} \psi_{\chi_{[-, 1,1]}}\right)}
$$

is a subcharacter of the Type 0 component of $\chi_{\left[1,1^{2}, 1\right]} \downarrow_{P}^{G}$. Next, we consider the Type 0 component of $\mathrm{R}_{L}^{G}\left(\chi_{[1,1,1]}\right) \downarrow_{P}^{G}$. We have

$$
\mathrm{R}_{L}^{G}\left(\chi_{[1,1,1]}\right)=\chi_{[2,1,1]}+\chi_{[1,2,1]}+\chi_{\left[1^{2}, 1,1\right]}+\chi_{\left[1,1^{2}, 1\right]} .
$$

As before, we see from the degrees that conjugation with $t$ fixes $\chi_{[1,1,1]}$. Hence, we get from (4.8) that $\mathrm{R}_{L}^{G}\left(\chi_{[1,1,1]}\right) \downarrow_{P}^{G}={ }^{1} \psi_{\chi_{[1,1,1]}}+{ }^{s} \chi_{[1,1,1]} \downarrow_{R Q_{K}}^{s} \uparrow_{R Q_{K}}^{P}+\chi_{[1,1,1]} \uparrow_{L}^{P}$. Via Theorems 7.1 and 4.3(b), (c) and (d), we can compute the Type 0 component of ${ }^{s} \chi_{[1,1,1]} \downarrow_{R Q_{K}}{ } \uparrow_{R Q_{K}}^{P}$ and get

$$
{ }^{0} \psi_{\left({ }^{1} \psi_{\chi_{[1,-, 1]}}\right)}+{ }^{0} \psi_{\left({ }^{1} \psi_{\chi_{[-, 1,1]}}\right)}+{ }^{0} \psi_{\Sigma}=2 \cdot{ }^{0} \psi_{\left({ }^{1} \psi_{\chi_{[1,-, 1]}}\right)}+2 \cdot{ }^{0} \psi_{\left({ }^{1} \psi_{\chi_{[-, 1,1}}\right)}+{ }^{0} \psi_{\left({ }^{0} \psi_{P_{P_{3}}}\right)} \cdot
$$

The Type 0 component of $\chi_{[1,1,1]} \uparrow_{L}^{P}$ can be determined with the help of Theorem 7.1 and Proposition 5.1 and we get

$$
\left.{ }^{0} \psi_{\left({ }^{1} \psi_{\chi_{[1,-, 1]}}\right)}+{ }^{0} \psi_{\left({ }^{1} \psi_{\chi_{[-, 1,1]}}\right)}+{ }^{0} \psi_{\left({ }^{0} \psi_{1_{P_{3}}}\right)}+{ }^{0} \psi_{\left({ }^{+} \psi_{L_{5}}\right)}\right) \cdot
$$

Together with (8.4), it follows that the Type 0 component of

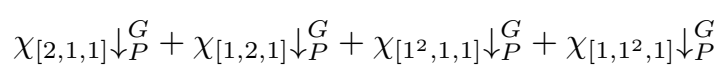

is $3 \cdot{ }^{0} \psi_{\left({ }^{1} \psi_{\chi_{[1,-, 1]}}\right)}+3 \cdot{ }^{0} \psi_{\left({ }^{1} \psi_{\chi_{[-, 1,1]}}\right)}+2 \cdot{ }^{0} \psi_{\left({ }^{0} \psi_{1_{P_{3}}}\right)}+{ }^{0} \psi_{\left({ }^{+} \psi_{1_{L_{5}}}\right)}$. Since we already know the Type 0 component of $\chi_{[2,1,1]} \downarrow_{P}^{G}+\chi_{[1,2,1]} \downarrow_{P}^{G}$, we see that the Type 0 component of $\chi_{\left[1^{2}, 1,1\right]} \downarrow_{P}^{G}+\chi_{\left[1,1^{2}, 1\right]} \downarrow_{P}^{G}$ is

$$
\left.2 \cdot{ }^{0} \psi_{\left({ }^{1} \psi_{\chi_{[1,-, 1]}}\right)}+2 \cdot{ }^{0} \psi_{\left({ }^{1} \psi_{\chi_{[-, 1,1]}}\right)}+2 \cdot{ }^{0} \psi_{\left({ }^{0} \psi_{1_{P_{3}}}\right)}+{ }^{0} \psi_{\left({ }^{+} \psi_{L_{5}}\right.}\right) \cdot
$$

Next, we consider the Type 0 component of $\mathrm{R}_{L}^{G}\left(\chi_{\left[1^{2},-, 1\right]}\right) \downarrow_{P}^{G}$. We have

$$
\mathrm{R}_{L}^{G}\left(\chi_{\left[1^{2},-, 1\right]}\right)=\chi_{[21,-, 1]}+\chi_{\left[1^{2}, 1,1\right]}+\chi_{\left[1^{3},-, 1\right]} .
$$

We have already seen above that conjugation with $t$ fixes $\chi_{\left[1^{2},-, 1\right]}$. Hence, we get from (4.8) that $\mathrm{R}_{L}^{G}\left(\chi_{\left[1^{2},-, 1\right]}\right) \downarrow_{P}^{G}={ }^{1} \psi_{\chi_{\left[1^{2},-, 1\right]}}+{ }^{s} \chi_{\left[1^{2},-, 1\right]} \downarrow_{R Q_{K}}^{s} \uparrow_{R Q_{K}}^{P}+\chi_{\left[1^{2},-, 1\right]} \uparrow_{L}^{P}$. Via Theorems 7.1 and $4.3(\mathrm{~b}),(\mathrm{c})$ and (e), we can compute the Type 0 component of ${ }^{s} \chi_{\left[1^{2},-, 1\right]} \downarrow_{R Q_{K}} P \uparrow_{R Q_{K}}^{P}$ and get

$$
{ }^{0} \psi_{\left({ }^{1} \psi_{\chi_{[1,-, 1]}}\right)}+{ }^{0} \psi_{\Sigma}={ }^{0} \psi_{\left({ }^{1} \psi_{\chi_{[1,-, 1]}}\right)}+{ }^{0} \psi_{\left({ }^{0} \psi_{1_{P_{3}}}\right)}+{ }^{0} \psi_{\left({ }^{1} \psi_{\chi_{[1,-, 1]}}\right)}+{ }^{0} \psi_{\left({ }^{1} \psi_{\chi_{[-, 1,1]}}\right)} .
$$

The Type 0 component of $\chi_{\left[1^{2},-, 1\right]} \uparrow_{L}^{P}$ can be determined via Theorem 7.1 and Proposition 5.1.

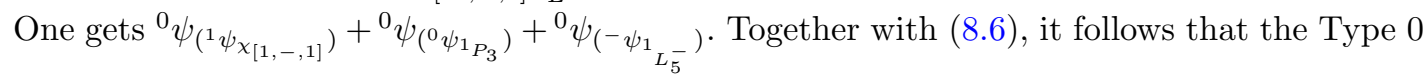
component of $\chi_{[21,-, 1]} \downarrow_{P}^{G}+\chi_{\left[1^{2}, 1,1\right]} \downarrow_{P}^{G}+\chi_{\left[1^{3},-, 1\right]} \downarrow_{P}^{G}$ is

$$
3 \cdot{ }^{0} \psi_{\left({ }^{1} \psi_{\chi_{[1,-, 1]}}\right)}+{ }^{0} \psi_{\left({ }^{1} \psi_{\chi_{[-, 1,1]}}\right)}+2 \cdot{ }^{0} \psi_{\left({ }^{0} \psi_{1_{P_{3}}}\right)}+{ }^{0} \psi_{\left(-\psi_{L_{L_{5}}}\right)} \cdot
$$

Since we already know the Type 0 component of $\chi_{[21,-, 1]} \downarrow_{P}^{G}$, we see that the Type 0 component of $\chi_{\left[1^{2}, 1,1\right]} \downarrow_{P}^{G}+\chi_{\left[1^{3},-, 1\right]} \downarrow_{P}^{G}$ is

$$
2 \cdot{ }^{0} \psi_{\left({ }^{1} \psi_{\chi_{[1,-, 1]}}\right)}+{ }^{0} \psi_{\left({ }^{1} \psi_{\chi_{[-, 1,1]}}\right)}+2 \cdot{ }^{0} \psi_{\left({ }^{0} \psi_{1_{P_{3}}}\right)}+{ }^{0} \psi_{\left(-\psi_{L_{L_{5}}}\right)} \cdot
$$


Equation (8.7) implies that ${ }^{0} \psi_{\left({ }^{+} \psi_{L}\right)}$ ) is no constituent of $\chi_{\left[1^{2}, 1,1\right]} \downarrow_{P}^{G}$ and hence it follows from (8.5) that ${ }^{0} \psi_{\left({ }^{+} \psi_{L_{5}}\right)}$ is a constituent of $\chi_{\left[1,1^{2}, 1\right]} \downarrow \stackrel{G}{G}$. Hence,

$$
{ }^{0} \psi_{\left({ }^{0} \psi_{P_{3}}\right)}+{ }^{0} \psi_{\left({ }^{1} \psi_{\chi_{[1,-, 1]}}\right)}+{ }^{0} \psi_{\left({ }^{1} \psi_{\chi_{[-, 1,1]}}\right)}+{ }^{0} \psi_{\left({ }^{+} \psi_{L_{L_{5}^{+}}}\right)}
$$

is a subcharacter of the Type 0 component of $\chi_{\left[1,1^{2}, 1\right]} \downarrow_{P}^{G}$. Comparing degrees with Table 10, we see that (8.8) is the Type 0 component of $\chi_{\left[1,1^{2}, 1\right] \downarrow}$. Now the Type 0 components of $\chi_{\left[1^{2}, 1,1\right]} \downarrow_{P}^{G}$ and $\chi_{\left[1^{3},-, 1\right]} \downarrow_{P}^{G}$ follow from (8.5) and (8.7). This gives the entries in rows 7, 8 and 10 of Table 9 and completes the proof of the theorem.

Remark 7. The proof of Theorem 8.1 gives partial information on the Type + and Type components of the restrictions of the unipotent characters of $G=\mathrm{SO}_{7}(q)$ to the maximal parabolic subgroup $P=P_{7}$. In particular, we see that the restrictions of $[3,-, 1],[21,-, 1]$ and $[1,-, 3]$ to $P$ do not have any constituents of Type + and that the Type + component of $[2,1,1] \downarrow_{P}^{G}$ is ${ }^{+} \psi_{1_{L^{+}}}$. Furthermore, the restrictions of $[3,-, 1],[2,1,1],[-, 3,1]$ and $[1,2,1]$ to $P$ do not have any constituents of Type - and the Type - component of $[21,-, 1] \downarrow_{P}^{G}$ is ${ }^{-} \psi_{1_{L^{-}}}$.

The constituents of Type $+/-$ are parameterized by irreducible characters of the groups $\mathrm{GO}_{4}^{ \pm}(q)$. At present, there is only limited information on the irreducible characters of these groups. The character tables of these groups and the remaining Type $+/-$ components will be treated in a forthcoming project.

Acknowledgements. We thank Frank Lübeck for fruitful discussions and for providing the unipotent characters of $\mathrm{SO}_{5}(q)$ and $\mathrm{SO}_{7}(q)$. We also thank Gerhard Hiss for many insightful discussions and the referee for useful comments.

\section{References}

1. J. AN and G. HIss, 'Restricting the Steinberg character in finite symplectic groups', J. Group Theory 9 (2006) 251-264.

2. J. AN and G. Hiss, 'Restricting unipotent characters in finite symplectic groups', Comm. Algebra 39 (2011) 1104-1130.

3. R. W. CARTer, Finite groups of Lie type - conjugacy classes and complex characters (John Wiley, New York, 1985).

4. J. Conway, R. T. Curtis, S. P. Norton, R. A. Parker and R. A. Wilson, Atlas of finite groups (Oxford University Press, Oxford, 1985).

5. H. Enомото, 'The characters of the finite symplectic group $\operatorname{Sp}_{4}(q), q=2^{f}$, Osaka J. Math. 9 (1972) $75-94$.

6. M. Geck, G. Hiss, F. Lübeck, G. Malle and G. Pfeiffer, 'CHEVIE - A system for computing and processing generic character tables for finite groups of Lie type, Weyl groups and Hecke algebras', Appl. Algebra Engrg. Comm. Comput. 7 (1996) 175-210.

7. R. M. Guralnick and P. H. Tiep, 'Cross characteristic representations of even characteristic symplectic groups', Trans. Amer. Math. Soc. 356 (2004) 4969-5023; (electronic).

8. F. Himstedt and F. Noeske, 'Decomposition numbers of $\mathrm{SO}_{7}(q)$ and $\mathrm{Sp}_{6}(q)$ ', J. Algebra 413 (2014) $15-40$.

9. G. Hiss and R. Kessar, 'Scopes reduction and Morita equivalence classes of blocks in finite classical groups', J. Algebra 230 (2000) 378-423.

10. I. M. IsAacs, Character theory of finite groups (Dover, New York, 1994) (reprint).

11. N. Jacobson, Basic algebra I, 2nd edn (W. H. Freeman, New York, 1985).

12. A. Schaeffer Fry, 'Cross-characteristic representations of $\operatorname{Sp}_{6}\left(2^{a}\right)$ and their restrictions to proper subgroups', J. Pure Appl. Algebra 217 (2013) 1563-1582.

13. T. Schmölzer, 'Restricting the Steinberg character in finite orthogonal groups', Diplomarbeit, RWTH Aachen University, 2012.

14. D. E. TAYLOR, The geometry of the classical groups, Sigma Series in Pure Mathematics 9 (Heldermann, Berlin, 1992). 
Frank Himstedt

Technische Universität München

Zentrum Mathematik - M11

Boltzmannstr. 3

85748 Garching

Germany

himstedt@ma.tum.de
Felix Noeske

Lehrstuhl D für Mathematik

RWTH Aachen

Templergraben 64

52062 Aachen

Germany

Felix.Noeske@math.rwth-aachen.de 